\title{
A Spin Fluctuation Model for $d$-wave Superconductivity
}

\author{
Andrey V. Chubukov ${ }^{1}$, David Pines ${ }^{2}$, and Jörg Schmalian ${ }^{3}$ \\ 1 Department of Physics, University of Wisconsin, Madison, WI 53706 \\ 2 Institute for Complex Adaptive Matter, University of California, T-Division, Los Alamos National Laboratory, Los Alamos, \\ NM 87545 and Department of Physics, University of Illinois at Urbana-Champaign, Urbana, Il 61801 \\ 3 Department of Physics and Astronomy and Ames Laboratory, Iowa State University, Ames, IA 50011
}

(October 23, 2018)

\begin{abstract}
We review the results of the spin-fermion model for correlated electron materials that are sufficiently close to an antiferromagnatic instability that their staggered static magnetic susceptibility in the normal state is large compared to that found in a conventional Fermi liquid. We demonstrate that for such materials magnetically-mediated superconductivity, brought about by the exchange of spin fluctuations, is a viable alternative to conventional phonon-mediated pairing, and leads to pairing in the $d_{x^{2}-y^{2}}$ channel. If the dominant interaction between quasiparticles is of electronic origin and, at energies much smaller than the fermionic bandwidth, can be viewed as being due to the emission and absorption of a collective, soft spin degree of freedom, the low-energy physics of these materials is accurately described by the spin-fermion model. The derived dynamic magnetic susceptibility and quasiparticle interaction coincide with the the phenomenonological forms used to fit NMR experiments and in earlier Eliashberg calculations. In discussing normal state properties, the pairing instability and superconducting properties, we focus our attention on those materials that, like the cuprate, organic, and some heavy electron superconductors, display quasi-two dimensional behavior. In the absence of superconductivity, at sufficiently low temperatures and energies, a nearly antiferromagnetic Fermi liquid is unconventional, in that the characteristic energy above which a Landau Fermi liquid description is no longer valid is not the Fermi energy, but is the much smaller spin-fluctuation energy, $\omega_{\text {sf }}$. For energies (or temperatures) between $\omega_{\text {sf }}$ and the Fermi energy, the system behavior is quite different from that in a conventional Fermi liquid. Importantly, it is universal in that it is governed by just two input parameters -an effective spin-fermion interaction energy that sets the overall energy scale, and a dimensionless spin-fermion coupling constant that diverges at the antiferromagnetic quantum critical point. We discuss the pairing instability cased by the spin-fluctuation exchange, and "fingerprints" of a spin mediated pairing that are chiefly associated with the emergence of the resonance peak in the spin response of a $d$-wave superconductor. We identify these fingerprints in spectroscopic experiments on cuprateb superconconductors. We conclude with a discussion of open questions associated primarily with the nature of the pseudogap state found in underdoped cuprates.
\end{abstract}


Contents

I Introduction and Overview

II $\quad$ Spin-fermion model

A Physical motivation of the spin fermion model

B Weak coupling approach to the pairing

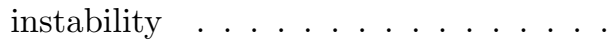

III Summary of strong coupling theory for electron phonon pairing

IV $\quad$ Strong coupling approach to spinfermion interaction 15

A Normal state behavior . . . . . . . 15

B The $d_{x^{2}-y^{2}}$ pairing instability temper-

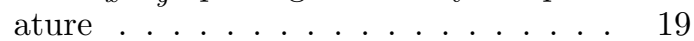

C Superconducting state. . . . . . . . 20 1 Generalized Eliashberg equations . 20 2 Solution of the Eliashberg equations: 22

\begin{tabular}{lll}
\hline & Fingerprints of spin fermion pairing & 24
\end{tabular}

A The physical origin of the effect . . . . 24

B Similarities and discrepancies between $d$ - and $s$-wave superconductors . . . 25

C The spectral function . . . . . . . 26

D The density of states . . . . . . . . . . . . . 28

\begin{tabular}{ll|lll}
\hline E & SIS tunneling & . . . . . . . . . . . . . & 29
\end{tabular}

F Optical conductivity and Raman response 31

VI Comparison with the experiments on cuprates 33

A Parameters of the model . . . . . . . . 33

B The normal state . . . . . . . . . . 34

1 The spectral function: . . . . . . . . 35

2 The optical conductivity . . . . . . 35

C The superconducting state . . . . . . . 36

1 The spin susceptibility . . . . . . . 36

2 The spectral function: . . . . . . . . . 37

3 The density of states: . . . . . . . . 38

4 SIS tunneling: .......... . . . 38

5 Optical and Raman response: . . . 39

D Experimental facts that we cannot yet

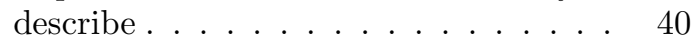

E $\quad$ Phase diagram . . . . . . . . . . . . 41

VII Conclusions

43

VIII Acknowledgments

\section{INTRODUCTION AND OVERVIEW}

2

The identification of the microscopic mechanisms responsible for superconductivity and the nature of the superconducting pairing state continues to represent one of the most exciting theoretical challenges in theoretical physicst. In the so-called conventional superconductors, at frequencies less than or comparable to the Debye frequency, the attractive phonon-induced interaction between electrons wins gut over the repulsive screened Coulomb interaction? and brings about superconductivity 3 . The pairing of electrons in the superconducting state is in an $s$-wave channel. The primacy of phonon-induced interaction in conventional superconductors has been demonstrated with great clarity. The phonon density of states, obtained by inelastic neutron scattering experiments, and the spectrum of the bosons which mediate pairing, as deduced from tymeling experiments, agree very well in systems like $\mathrm{Pb}$ th. In addition to the isotope effect 6 , this comparison of two independent experiments is generally considered to be a very reliable proof of a phonon-mediated pairing state.

The analysis of the tunneling data relies heavily on the existence of strong coupling effects in the quasiparticle density of states and assumes the validity of the Eliashberg approach to superconductivity Eliashberg theory for conventional superconductors is extremely robust due to the decoupling of typical electron and phonon time scales caused by the small ratio of the velocity of sound and the Fermi velocity. This smallness also implies that the Debye frequency is much smaller than the Fermi energy, and hence the quasiparticles that participate in the pairing are low-energy quasiparticles, located in the near vicinity of the Fermi surface. Landau $\mathrm{g}$ showed that the low energy properties of a normal Fermi liquid are characterized by a small number of parameters and are independent of the details of the underlying lattice Hamiltonian. Both, the normal and superconducting states of a conventional superconductor may be viewed as protected states of matter 10 , states whose generic low energy properties, insensitive to microscopic details at large energy, are determined by a higher organizing principle. In this view, in conventional superconductors, the superconducting transition marks a transition from a Landau Fermi liquid quantum protectorate to a BCS quantum protectorate. The success of the BCS-Eliashberg theory for conventional phonon mediated superconductors is almost unique for interacting many body systems.

It is well known however that the pairing state in the Bardeen-Cooper-Schrieffer theory does not need to be caused by the interaction between electrons and lattice vibrations. Generally there are two distinct classes of theories of unconventional pairing. The first, and more conservative approach is to replace phonons by another collective bosonic excitation of the solid. This approach successfully describes the physics of superfluid ${ }^{3} \mathrm{He} 12$, where the intermediate bosons are failed ferromagnetic spin 
fluctuations (ferromagnetic paramagnons); a large value of the ferromagnetic correlation length is not required in view of the Kohn-Luttinger effect 11 . This magneticallymediated interaction causes pairing in a state with angular momentum $l=1$ ( $p$-wave pairing) and leads to a rich phase diagram and a large class of new physical phenomena 12 44. Magnetically-mediated superconductivity has been proposed for various organic and heavy fermion superconductors by a number of authors 1521 .

A second approach to unconventional pairing is more phenomenological, and is based on the assumption that the superconducting condensation energy is not determined by the attractive interaction mediated by some boson but rather by the energy gain due to feedback effects associated with pairing. The latter may, in principle, occur even for a purely repulsive pairing interaction. In general, this approach assumes a non-Fermi-liquid behavior in the normal state. Twexamples are the interlayer tunneling model of Refs.22.23 and the mid-infrared model of Ref. 24. In both models, the expectation value of the Hamiltonian is drastically different in the superconducting and the normal states, and the energy gain due to pairing apparently cannot be traced back to some boson-mediated attraction.

The two approaches to unconventional pairing are not necessarily in contradiction with each other. First, at strong coupling, the pairing interaction mediated by a low-energy bosonic mode is highly retarded and is a complex function of frequency, so it is not straightforward to determine whether it is repulsive or attractive. Second the pairing obviously changes the form of the fermionic self-energy and hence affects the kinetic energy. And third, if the bosonic mode is itself made out of fermions, then the propagator of this mode also changes when the system becomes superconducting. This change affects the potential energy of the system. Which of the two effects is larger depends on the details of the system behavior, but in any case, there are clear similarities between a strong-coupling theory which involves a pairing boson, and a scenario based entirely on energy gain due to feedback from pairing.

In what follows we adopt the first approach and investigate the role of failed antiferromagnetic spin fluctuations (antiferromagnetic paramagnons) as a possible cause for both anomalous normal state behavior and unconventional i.e. non $s$-wave superconductivity. This approach is chiefly motivated by the physics of the high temperature cuprate superconductors which have been shown to exhibit both highly anomalous normal state behavior and an unconventional pairing state with angular momentum $l=2$ (d-wave pairing) 25 27. As may be seen in Fig.11, the materials with the highest $T_{c}$ are located reasonably close to an antiferromagnetic state and have been shown in nuclear magnetic resonance and inelastic neutron scattering experiments to exhibit significant antiferromagnetic correlations in the paramagnetic state. 33 We will show in agreement with the calculations of Monthoux et al.31.32, that in a quasi two-dimensional material where those correlations are significant (e.g. a spin correlation length larger than a lattice constant) the normal state behavior is anomalous while for Fermi surface parameters appropriate for the cuprates, one always gets a $\mathrm{d}_{x^{2}-y^{2}}$ superconducting pairing state. We discuss other materials below, following a brief historical overview of the developments in the spin-fluctuation approach over the last decade. References to earlier works can be found in the papers cited below.

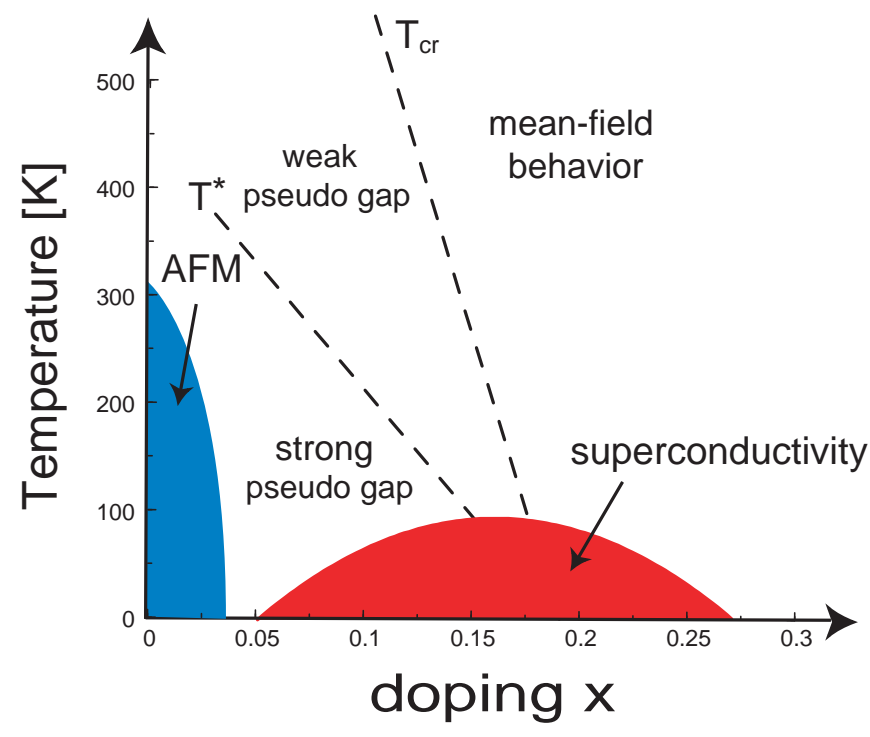

FIG. 1. Generic phase diagram of high temperature superconducting cuprates. The thermodynamic phases (antiferromagnetic at low doping and superconducting at higher doping) are depicted by the shaded regions. The remaining lines are either phase transitions or crossovers, visible in a variety of experiments.

A $d_{x^{2}-y^{2}}$ pairing state in two dimensions due to the exchange of near-antiferromagnetic spin fluctuations was found in the gletailed Hubbard model calculations of Bickers et al. $1 \mathrm{Q}$. For parameters believed to be relevant for cuprates in 1987, the superconducting transition temperature was comparatively low $(<40 \mathrm{~K})$ under what seemed to be optimal conditions. Furthermore, $\mathrm{T}_{c}$ decreased as one increased the planar hole concentration from a low level, in contrast to experiment. These results, when taken together with the early penetration-depth experiments that supported an s-wave pairing state, were responsible for the fact that the magnetic mechanism and $d_{x^{2}-y^{2}}$ pairing had been abandoned by most of the high temperature superconductivity community by the end of 1989 (Bedell et al 39 ).

At about this time, theoretical groups in Tokyd29, 30 and Urbana 13 . 22 independently began developing a semiphenomenological, macroscopic theory of spin-mediated pairing. Both groups assumed that the magnetic interaction between the planar quasiparticles was responsible for the anomalous normal state properties and found a superconducting transition to a $d_{x^{2}-y^{2}}$ pairing state at 
a significantly higher temperature than those-achieved using the Hubbard model. Moriya et al.29.30 used a self-consistent renormalization group approach to characterize the dynamic spin susceptibility. The resulting effective magnetic interaction between the planar quasiparticles was then used to calculate $\mathrm{T}_{c}$ and the normal state resistivity. Monthoux et al 32 did not attempt a first-principles calculation of the planar quasiparticle interaction. Rather, they turned to experiment and used quasiparticles whose spectra was determined by fits to band structure calculations and angular resolved photoemission spectroscopy (ARPES) experiments. The effective magnetic interaction between these quasiparticles was assumed to be proportional to a mean field dynamif spin susceptibility of the form developed by Millis et al. 34 that had been shown to provide an excellent description of NMR experiments on the $\mathrm{YBa}_{2} \mathrm{Cu}_{3} \mathrm{O}_{7-y}$ system 33, 34 .

Both groups followed up their initial weak coupling calculations with strong coupling (Eliashberg) calculations 3538 that enabled them to take into account lifetime effects brought about by the strong magnetic interaction. These calculations showed that $d_{x^{2}-y^{2}}$ superconductivity athigh $\mathrm{T}_{c}$ is a robust phenomenon. Monthoux and Pines 37 also found in a strong coupling calculation that they could obtain an approximately correct magnitude and temperature dependence of the planar resistivity of optimally doped $\mathrm{YBa}_{2} \mathrm{Cu}_{3} \mathrm{O}_{7-y}$ using the same coupling constant (and the same parameters to characterize the quasiparticle and spin spectrum) that had yielded a $\mathrm{T}_{c}$ of approximately $90 \mathrm{~K}$. They concluded that they had established a "proof of concept" for a nearly antiferromagnetic Fermi liquid (NAFL) description of the anomalous normal state behavior and a spin fluctuation mechanism for high temperature superconductivity. Referring back to Fig. 1, these calculations should apply to the right of the $T_{\mathrm{cr}}$-line, where the normal state is an unconventional Fermi liquid in which the characteristic energy above which quasiparticles loose their Fermi liquid behavior is of order of the spin fluctuation energy and low compared to the fermionic bandwidth.

Since their calculations unambiguously predicted a $d_{x^{2}-y^{2}}$ pairing state, Monthoux and Pines challenged the experimental community to find unambiguously the symmetry of the pairing state. At that time (1991-1992), only NMR Knight shift and $C u$ spin-lattice relaxation rate results supported $d_{x^{2}-y^{2}}$ pairing 40 44. However within the next year or so, the tide turned dramatically awayfrom s-wave pairing, with ARPFs 45 , penetration depth 46 and new NMR experiments 44 on the oxygen spin-lattice relaxation time and the anisotropy of the copper spinlattice relaxation time all supporting a $d_{x^{2}-y^{2}}$ state. The decisive experiments were the direct phase-sensitive tests of pairing symmetry garried out by Van Harlingen and his group in Urbane 25 as well as by Kirtley, Tsuei, and their collaboratorse.

In subsequent work on the spin-fluctuation mechanism a microscopic Hamiltonian approach to the problem was developed 50.51. It was shown that the low-energy physics of spin-mediated pairing is fully captured by a model that describes the interaction of low energy fermionic quasiparticles with their oyn collective spin excitations (the spin-fermion model)50.51. In particular, it was demonstrated that the phenomenological interaction between quasiparticles could be derived in a controllable way, even at strong coupling, by expanding either in the inverse number of hot spots in the Brillouin zone $(=8$ for the physical case), or in the inverse number of fermionic flavors. We discuss this theory in detail in Section 4. As will be seen there, the spin-fermion model contains only a small number of parameters. These uniquely determine system behavior that is fully universal in the sense that it does not depend on the behavior of the underlying electronic system at energies comparable to the fermionic bandwidth. It is therefore possible to verify its applicability by first using a few experimental results to determine these parameters, and then comparing the predictions of the resulting parameter-free theory with the larger subset of experimental results obtained at temperatures and frequencies which are much smaller than the fermionic bandwidth. A major prediction of the spin fermion model is that the upper energy scale for the Fermi liquid behavior progressively shifts down as the system approaches a quantum-critical point at $T=0$, and there emerges a large intermediate range of frequencies where, on the one hand, the system behavior is still a low-energy one and universal, and on the other hand, it is quantum-critical and not a Fermi liquid.

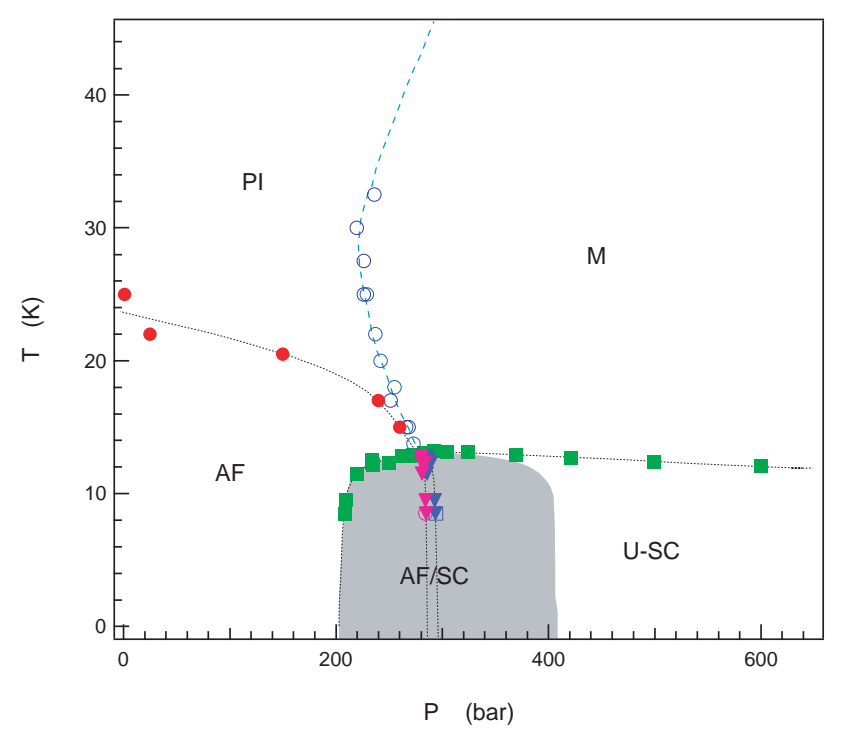

FIG. 2. The phase diagram of the layered organic superconductor $\kappa$ - $(\mathrm{ET})_{2} \mathrm{Cu}\left[\mathrm{N}(\mathrm{CN})_{2}\right] \mathrm{Cl}$ in the units of temperature and pressure (from Ref. 65). PI refers to a paramagnetic insulating regime, $\mathrm{M}$ to a metallic regime, $\mathrm{AF}$ to an antiferromagnetic regime, and SC to a superconducting regime. In the region AF-SC, superconductivity and antiferromagnetism co-exist. In the region U-SC, the system is an unconventional superconductor 
Cuprate superconductors are not the only candidate materials for spin fluctuation mediated pairing and non-Fermi liquid quantum protectorates. A number of organic superconductors are anisotropic quasi-twodimensional materials that exhibit many of the anomalies typical of a system with an unconventional pairing state. The phase diagram of a quasi two-dimensional organic compound $\kappa$-BEDT-TTF is shown in Fig. 2. One can see that, as in the case of the cuprates, the superconducting phase is found in the vicinity of an antiferromagnetic phase. Several groups 525 have used spinfluctuation theory to predict the position of nodes of the superconducting order parameter of these materials. An unconventional order-parameter with nodes of the gap is indeed supported by NMR 5058 , thermal conductivity 59 , millimeter transmission 61 and STM 62 experiments. However, the last two experiments seem to come to different conclusions as far as the position of the nodes is concerned. Ref.60 also finds nodes, but at a position that is not consistent with the prediction of a spin fluctuation inducedpairing state. Finally, penetration depth experiment 63 and recent specific heat data 64 appear to support a conventional s-wave gap. Given those contradictory experiments, whether quasi-two dimensional organic superconductors exhibit an unconventional pairing state is, as of this writing, an open question.

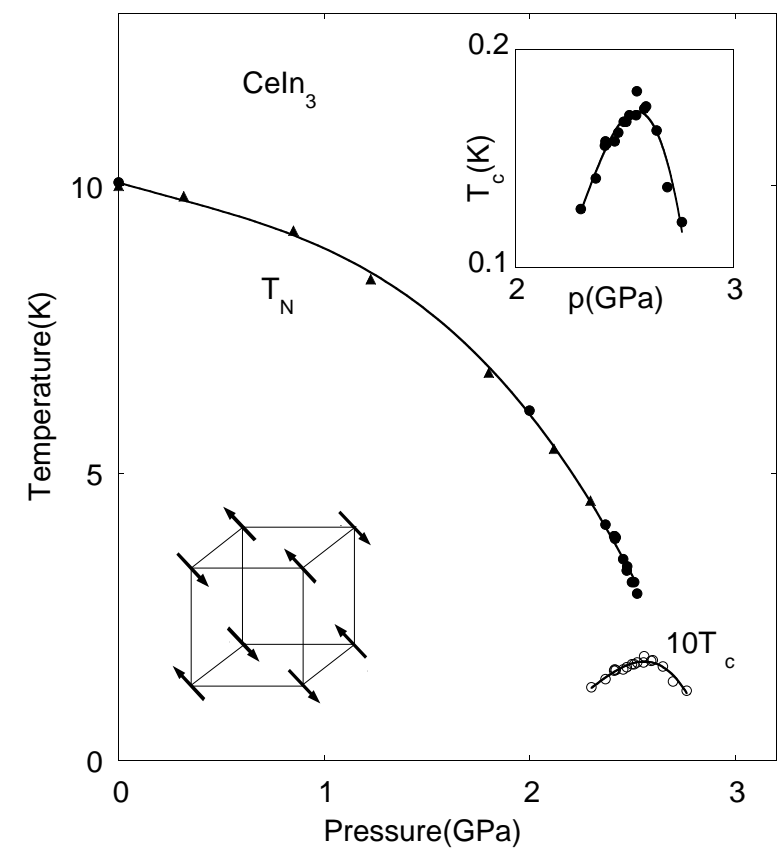

FIG. 3. The phase diagram of $\mathrm{CeIn}_{3}$ in the units of temperature and pressure (from Ref. 67).

Cerium-based heavy electron superconductors represent another class of strongly correlated electron superconductors for which a spin-fluctuation induced interaction between quasiparticles is a strong candidate for the superconducting mechanism. Examples include
$\mathrm{CeCu}_{2} \mathrm{Si}_{2} 6$, $\mathrm{CePd}_{2} \mathrm{Si}_{2}, \mathrm{CeIn}_{3} 6$ and the newly discorered 1-1-5 materials $\mathrm{CeXIn}_{5}$ with $\mathrm{X}=\mathrm{Co}, \mathrm{Rh}$ and $\mathrm{Ir} 68$ or mixtures thereof. As may be seen in the phase diagrams of Fig. 3 and Fig.4, all these materials are close to antiferromagnetism, with superconductivity occurring close to the critical pressure or alloy concentration at which the magnetic ordering disappears. Moreover, thermal conductivity measurements of $\mathrm{CeCoIn}_{5}$, which becomes superconducting at $2.4 \mathrm{~K}$ at ambient pressures the highest known value of $T_{c}$ for a heavy fermion based system - strongly support a superconducting gap with nodes along the $( \pm \pi, \pm \pi)$ directions, as found in a $\mathrm{d}_{x^{2}-y^{2}}$ pairing state 70 . Another exciting aspect of these systems is that by changing the relative compositions of Ir and Rh in 1-1-5 materials $\mathrm{CeRh}_{1-\mathrm{x}} \operatorname{Ir}_{\mathrm{x}} \mathrm{In}_{5}$, one can move the system from an antiferromagnetic to a superconducting state at ambient pressure.

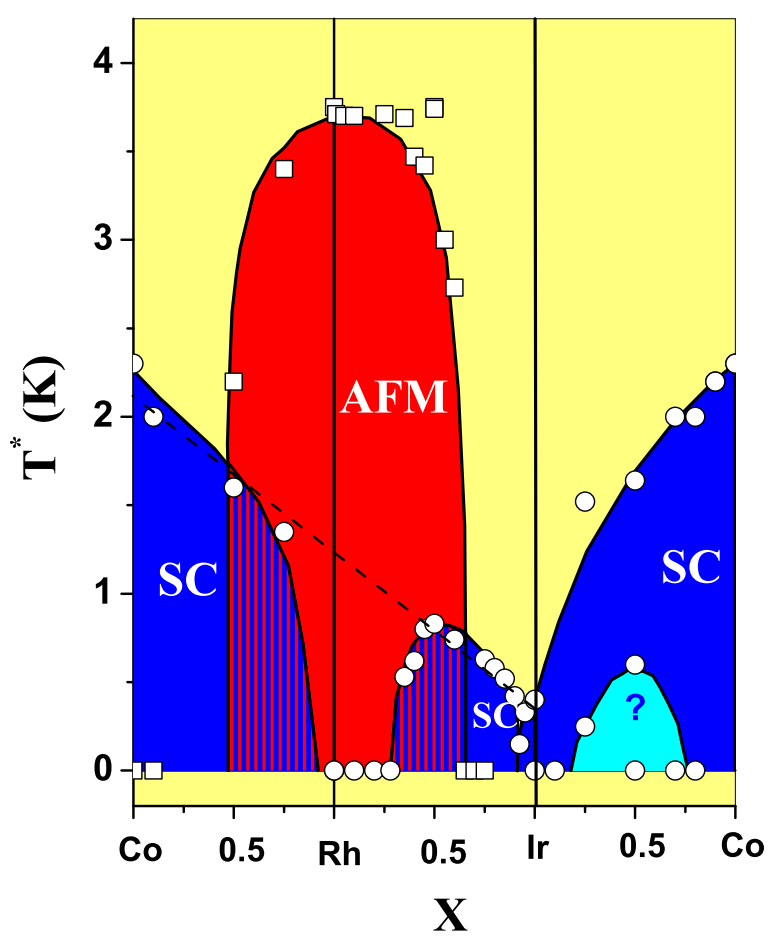

FIG. 4. The phase diagram of $\mathrm{Ce}_{2} \mathrm{X} \mathrm{In}_{5}$ with $\mathrm{X}=\mathrm{Co}, \mathrm{Rh}$, and Ir in the units of temperature and doping (from Pagliuso et al. 99 ).

Another widely studied material in which pairing is possibly due to spin fluctuation exchange is $\mathrm{Sr}_{2} \mathrm{RuO}_{4} 7174$, where NMR Knight shift experiments 75 and spin-polarized neutron scattering measurements 76 reveal that the spin susceptibility is unchanged upon entering the superconducting state, consistent with spintriplet superconductivity.

In summary, the cuprates, the 1-1-5 heavy fermion materials and the layered organic superconductors are 
strongly correlated materials that exhibit unconventional normal state and superconducting behavior, while the superconducting phases are located in the vicinity of magnetic instabilities in their corresponding phase diagrams. It is then quite natural to assume that in all three cases magnetic interactions play a dominant role in the pairing.

The presence of antiferromagnetic and superconducting regions in the phase diagram raises the question of whether antiferromagnetism and superconductivity should be treated on equal footing in a spin fluctuation approach. If they should, the theoretical analysis would be complex. Fortunately, this is not the case, at least as long as the characteristic energy scales for the magnetic interactions are smaller than the fermionic bandwidth. The point is that superconductivity is generally a lowenergy phenomenon associated with fermions in the near vicinity of the Fermi surface. On the other hand, antiferromagnetism originates in fermions with energies comparable to the bandwidth. Perhaps the easiest way to see this is to formally compute the static spin susceptibility in the random phase approximation (RPA). An RPA analysis yields $\chi^{-1}(\mathbf{q}) \propto 1-g_{\mathrm{eff}}(\mathbf{q}) \Pi(\mathbf{q})$ where $g_{\mathrm{eff}}(\mathbf{q})$ is some effective interaction, and $\Pi(\mathbf{q})$ is the static spin polarization operator (a particle-hole bubble with Pauli matrices in the vertices). For an antiferromagnetic instability we need $g_{\text {eff }}(\mathbf{Q}) \Pi(\mathbf{Q})=1$. One can easily make sure, by evaluating $\Pi(\mathbf{Q})$ for free fermions, that the momentum/frequency integration in the particle-hole bubble is dominated by the upper energy limit that is the fermionic bandwidth. This implies that whether or not a system orders antiferromagnetically is primarily determined by high-energy fermions that are located far away from the Fermi surface, and hence the antiferromagnetic correlation length, that measures the proximity of a material to a nearby antiferromagnetic region in the phase diagram, should not be calculated but rather be taken as an input for any low-energy analysis. We discuss the practical meaning of this separation of energies in Section 4.

A more subtle but important issue is whether the dynamical part of the spin susceptibility should be considered simply as an input for a low-energy model (as in the case for phonons), or whether the spin dynamics is produced by the same electrons that are responsible for the superconductivity and hence needs to be determined consistently within the low-energy theory. The first issue one has to consider here is whether a oneband description is valid, i.e., whether localized electrons remain quenched near the antiferromagnetic instability and form a single large Fermi surface together with the cenduction electrons to which they are strongly coupled 77, 88, or whether the magnetic instability is accompanied by the un-quenching of local moments. In the latter case, the volume of the Fermi surface changes discontinuously at the magnetic transition and could e.g., cause a jump in the Hall coefficient 79 . The quenching versus un-quenching issue is currently amject of intensive debate in heavy fermion materials 79 .80. In cuprates the quenching versus un-quenching issue does not seem to play a role; it is widely accepted that the formation of Zhang-Rice singlet\$81 gives rise to a single electronic degree of freedom. Similarly, in organic materials, the charge transport in the metallic and superconducting parts of the phase diagram is due to the same missing electrons in otherwise closed filled molecular orbital states. Whether or not the spin dynamics originates in low-energy fermions then reduces to the geometry of a single, large Fermi surface. For a Fermi surface with hot spots, connected by the wave vector at which the spin fluctuation spectrum peaks, the low-energy spin dynamics is dominated by a process in which a collective spin excitation decays into a particle-hole pair. By virtue of energy conservation, this process involves fermions with frequencies comparable to the frequency of a spin excitation. Consequently, the spin dynamics is not an input. If, however, the Fermi surface does not contain hot spots, spin damping is forbidden at low-energies and spin fluctuations are magnon-like propagating excitations. It is easy to show that in the latter situation, the full form of the spin propagator comes from particle-hole excitations at energies comparable to the bandwidth and therefore should be considered as an input for the low-energy theory.

In this chapter we consider in detail the scenario in which the Fermi surface contains hot spots and the spin damping by quasiparticles is allowed. Our approach to the cuprates is largely justified by the results of extensive ARPES and NMR and neutron measurements that indicate that the Fermi surface possesses hot spots, and that spin excitations are overdamped in the normal state.

Whether or not spin fluctuations are overdamped is also of significant conceptual importance for spin mediated pairing, since this mechanism requires that quasiparticles be strongly coupled to the collective spin excitation mode. At first glance, the undamped (magnon) form of the spin propagator appears more favorable for spin-mediated pairing than the overdamped form. Indeed, if one assumes that the spin-mediated interaction is just proportional to the spin susceptibility, the magnonlike form is preferable. By the Goldstone theorem, in the antiferromagnetically ordered state, the transverse spin susceptibility $\chi(\mathbf{q})$ (that yields an attraction in the $d_{x^{2}-y^{2}}$ channel) even diverges as $\mathbf{q}$ approaches the antiferromagnetic momentum $\mathbf{Q}$, hence the $d$-wave attraction appears to be the strongest. This reasoning, hayever, is incorrect. Schrieffer and his collaborators 823 and others 84 have shown that the Goldstone modes of an ordered antiferromagnet cannot give rise to a strong $d$-wave pairing because the full spin mediated interaction is the product of the spin susceptibility and the square of the fully renormalized coupling constant between fermions and magnons. The latter vanishes in the ordered SDW state at $\mathbf{q}=\mathbf{Q}$ and this effect exactly compensates the divergence of the static susceptibility. The vanishing of the effective coupling is a consequence of the Adler principle which states that true Goldstone modes 
always decouple from other excitations in a system 86 .

Schrieffer later argued 83 that the near cancellation between the enhancement of the spin susceptibility and the reduction of the effective magnon-fermion interaction persists in the paramagnetic state as long as spin fluctuations remain propagating excitations This would substantially reduce (although not eliminate87) the spinmediated $d$-wave attraction. This argument is however inapplicable to overdamped spin fluctuations. These are not Goldstone modes although they become gapless at the magnetic instability. Goldstone modes appear only in the ordered state at the smallest $\mathbf{q}-\mathbf{Q}$ values $\mathbf{5 5}$. For near-gapless, but overdamped spin excitations, the Adler principle does not work. After all, the damping itself is due to the strong coupling of the collective mode to fermions. Consequently, the spin-fermion vertex does not vanish at the magnetic transition and hence cannot cancel out the enhancement of the $d$-wave interaction due to the increase of the spin susceptibility near $\mathbf{Q}$. Thus, overdamped spin fluctuations are better for spin-mediated pairing than magnon-like excitations.

Another aspect of the fact that spin dynamics is made out of low-energy fermions is that the retarded interaction which causes the pairing changes when fermions acquire a superconducting gap. This feedback from quasiparticle pairing on the form of the pairing interaction distinguishes pairing mediated by overdamped spin fluctuations from conventional phonon induced pairing. In the latter the bosonic propagator is an input and is only very weakly affected by the opening of the gap in the quasiparticle spectrum. We will discuss in detail how feedback forces one to go beyond an approach in which one solely replaces a phonon by a spin fluctuation, and requires that one consistently calculates the spin dynamics at low energies. While doing this is a theoretical challenge, the approach is appealing since it reduces the number of unknown parameters in the problem. In particular, we will see that in the superconducting state, the propagator of spin fluctuations acquires the same form as for optical phonons, but the collective mode that is the analog of the phonon frequency is fully determined by the superconducting gap and the normal state spin damping. This gives rise to new, unique "fingerprints" of spin mediated pairing, whose presence can be checked experimentally.

What is the role of dimensionality? As noted above, many of the candidates for spin-mediated pairing are strongly anisotropic, quasi-two dimensional systems. This not only holds for the cuprates, but also for a large class of organic superconductors. Also, heavy fermion superconductors such as $\mathrm{CeCoIn}_{5}$ display a considerable spatial anisotropy. On the other hand, $\mathrm{CeIn}_{3}$ and to a lesser extent $\mathrm{CeCu}_{2} \mathrm{Si}_{2}$ do not display appreciable quasi two-dimensionality in their electronic properties. The dimensionality of the electronic system is important to the spin fluctuation model for both normal state and superconducting behavior. We will see that the dynamics of the fermions in the normal state is very differently affected by antiferromagnetic spin fluctuations in two and in three dimensional systems. While in the latter case only small (logarithmic) corrections to the ideal Fermi gas behavior occur in the vicinity of hot spots, we shall see that in $2 \mathrm{~d}$ systems, the strong interaction between fermions and spins gives rise to non-Fermi liquid, diffusive behavior of low energy fermions as the quantum critical point is approached. The importance of dimensionality for superconductivity has been emphasized by Monthoux and Lonzarich 74 who have shown that it exerts a considerable influence on the superconducting transition temperature. They pointed out that in three dimensions one cannot avoid repulsive contributions to the pairing interaction in choosing a pairing state with nodes, so that the same spin-mediated quasiparticle interaction is far less effective in bringing about superconductivity in three dimensions than in two.

Since the non-Fermi-liquid behavior of fermionic quasiparticles extends down to progressively lower frequencies as one approaches the magnetic transition at $T=0$, one can inquire whether pairing near this quantum-critical point is caused by the fermions at the lowest energies that are still coherent, or comes from those at higher energies (that are still smaller than the bandwidth) that display non-Fermi-liquid behavior. If only coherent fermions are involved in the pairing, then according to McMillan's extension of the BCS theory 88 , the resulting superconducting transition temperature, $T_{c}^{\mathrm{FL}}$, is comparable to the upper energy cutoff of the Fermi liquid regime, and thus will be of the order of the spin fluctuation energy. This energy vanishes at the critical point, and therefore magnetic criticality is unaffected by pairing (see the left panel in Fig.5). If, however, "non-Fermi-liquid" fermions can give rise to a pairing instability, then the onset temperature of this instability in the particle-particle channel, that we will identify with $T_{c r}$ in the phase diagram of Fig.1 generally scales with the upper cutoff energy for the quantum-critical behavior and remains finite at criticality $122,123,102$. In this situation, the quantum critical point is necessarily surrounded by a dome beneath which pairing correlations cannot be neglected as shown in the central panel of Fig.5. The critical behavior inside and outside the dome is different, and the "primary" critical behavior (which gives rise to pairing) can only be detected outside the dome. We will demonstrate below that the incoherent pairing temperature saturates at a finite value when the magnetic correlation length diverges. Furthermore, for parameters relevant to cuprates at low doping, this temperature is of order of the magnetic exchange interaction $J$, i.e., it is not small.

A related issue is whether the pairing instability at $T_{c r}$ implies the onset of true superconductivity (i.e., $T_{c r} \equiv T_{c}$ ), or whether it marks the onset of pseudogap behavior. In the latter scenario, for which we will see there is considerable experimental support, fluctuations prevent a superfluid density $\rho_{s}$ from developing a nonzero value until one reaches a much smaller $T_{c}$, and the paired incoherent fermions do not participate in superconductivity. The phase between $T_{c r}$ and $T_{c}$ would then be a 
newstate of matter, the pseudogap state. Abanov et al. 102 conjectured that a pseudogap regime is a universal feature of the spin fluctuation scenario, as below $T_{c r}$, quasiparticles that are paired into singlets still remain incoherent and cannot carry a supercurrent. True superconductivity is reached only at much smaller $T_{c}$ where the systems recovers coherent, Fermi-liquid behavior (see the right panel in Fig.5).
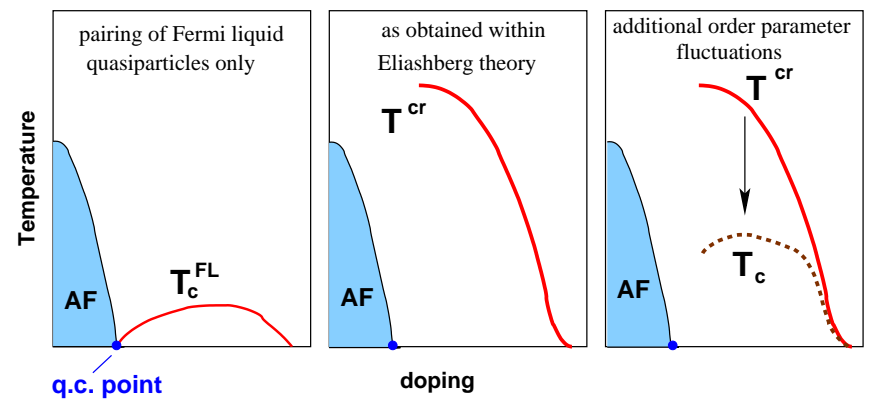

FIG. 5. The candidate phase diagrams in the units of temperature and doping of a one-band electronic system near an antiferromagnetic quantum critical point. Left panel - the phase diagram for the hypothetical situation when only coherent, Fermi liquid quasiparticles contribute to the pairing (the McMillan theory applied to spin fluctuations). The antiferromagnetic and superconducting regions are completely decoupled. Central panel - the solution of the coupled set of the Eliashbergequations for the onset of spin-mediated pairing instability 122 . The solution shows that at strong coupling, the the pairing instability is predominantly produced by incoherent fermions, and the instability temperature remains finite at $\xi=\infty$. Right panel, the proposed phase diagram based on the solution of the Eliashberg equations below the pairing instabilitand general arguments about superconducting fluctuations 102 .

The term pseudogap was introduced by Friedel 145 to describe the fact that in the underdoped regime of the cuprates, the planar quasiparticles begin to develop a gap-like structure well above $T_{c}$. This behavior was first seen in Knight, shift measurements of the uniform spin susceptibility 143 , and later detected in almost all measured properties of underdoped cuprates. At present, the physics of the pseudogap phase in the underdoped cuprate superconductors is not yet fully understood and its origin continues to be an open question. We believe that the "magnetic scenario" for the pseudogap provides a reasonable explanation, but many details still need to be worked out. Some researchers on the other hand have suggested that the pseudogap phase emerges due to strong fluctuations of the phase of the superconducting order parameter.111 115 . Others suggest that the pseudogap phase may actually be a new phase of matter with ahidden order parameter associated with bond current.89.91 (most recently, this idea has been explored in detail by Chakravarty et al. 22 ). A somewhat more general phenomenological possibility discussed by several researchers is that there exists an additional quantum crit- ical pqintgf yet unknown origin slightly above optimal doping 9396 (a number of experiments suggest that this point is at doping concentration $x \approx 0.19$ ). The pseudogap and Fermi liquid phases are assumed to be to the left and to the right of this new quantum critical point, respectively.

Our main goal is to discuss in detail the "primary" quantum-critical behavior within the magnetic scenario and how it gives rise to pairing at $T_{c r}$. A detailed theory of the pseudogap state of high temperature superconductors is beyond the scope of this Chapter. However, in the interest of providing a base line against which to compare both experiment and future theoretical developments, we summarize the predictions of the spin-fermion model for the pseudogap in Section 6 and discuss other alternatives.

To spell out the expected regions of applicability to the superconducting cuprates (in doping and temperature) of the spin-fluctuation theory without pseudogap physics involved, we return to the candidate generic phase diagram in Fig.11. The two lines, $T_{c r}$ and $T^{*}$ determine distinct regimes of physical behavior. Above $T_{c r}$, pseudogap physics plays no role; the theory of a nearly antiferromagnetic Fermi liquid (NAFL) presented in this Chapter should be applicable for both the normal state and the superconducting state. Since $T_{c r}$ crosses $T_{c}$ near the optimal doping concentration, the theory with no pseudogap involved is roughly applicable at and above optimal doping (from an experimental perspective, optimally doped materials do show some pseudogap behavior, but only over a very limited temperature regime). For the overdoped and nearly optimally doped cuprates the transition is then from a nearly antiferromagnetic Fermi liquid to a BCS superconductor with $d_{x^{2}-y^{2}}$-pairing symmetry. We will argue in Section 6 that there is a great deal of experimental evidence that at and above optimal doping the normal state is indeed a NAFL, and that the pairing is of magnetic origin. It is also likely that the theory can also be extended into a so-called "weak pseudogap" regime between $T_{c r}$ and $T^{*} 98102$, but we will not discuss this issue here.

In Section 2 we introduce and motivate the spin fermion model that we use to study spin fluctuation induced pairing. We discuss the weak coupling approach to the pairing problem and the symmetry of the magnetically mediated pairing state. In Section 3 we review the main results and arguments used to justify Eliashberg theory for conventional phonon superconductors. In particular, we discuss the physical origin of the Migdal theorem that allows a controlled approach to phonon-induced pairing. In Section 4 we then analyze in detail the strong coupling theory for the spinfermion model. We first discuss the normal state properties of this model and calculate the low frequency dynamics of quasiparticles and spin fluctuations. We next consider spin-fluctuation induced superconductivity. We show that for magnetically-mediated superconductivity one can again analyze the pairing problem in controlled calculations that on the level of the equations involved 
resemble the Eliashberg equations for electron-phonon superconductivity 99 . 51 . We demonstrate that the actual physical origin of the applicability of a generalized Eliashberg approach for spin mediated pairing is qualitatively different from the phonon case, and is associated with the overdamped nature of the spin excitations. We solve the resulting equations in certain limits and investigate the role of quantum critical pairing. In Section 5 we present a general discussion of some of the observable fingerprints of spin fluctuation induced superconductivity, and in Section 6 we compare our results with experiments and discuss to what extent the fingerprints of spin mediated pairing have already been seen in optimally doped cuprate superconductors. In our concluding Section 7 we summarize our results and comment on several topics that are of interest for a further understanding of spin mediated pairing, including the extent to which our theory can be extended to address the physics of the pseudogap state in underdoped cuprates.

\section{SPIN-FERMION MODEL}

\section{A. Physical motivation of the spin fermion model}

We first discuss the formal strategy one has to follow to derive an effective low-energy model from a microscopic Hubbard-type Hamiltonian with a four fermion interaction:

$$
\begin{aligned}
\mathcal{H} & =\sum_{\mathbf{k}, \alpha} \varepsilon_{\mathbf{k}} \psi_{\mathbf{k}, \alpha}^{\dagger} \psi_{\mathbf{k}, \alpha} \\
& +\sum_{\mathbf{k}_{\mathbf{i}}, \alpha_{i}} U_{\mathbf{k}_{1}, \mathbf{k}_{2}, \mathbf{k}_{3}, \mathbf{k}_{4}}^{\alpha_{1}, \alpha_{2}, \alpha_{3}, \alpha_{4}} \psi_{\mathbf{k}_{1}, \alpha_{1}}^{\dagger} \psi_{\mathbf{k}_{2}, \alpha_{2}}^{\dagger} \psi_{\mathbf{k}_{3}, \alpha_{3}} \psi_{\mathbf{k}_{4}, \alpha_{4}}
\end{aligned}
$$

Here $U_{\mathbf{k}_{1}, \mathbf{k}_{2}, \mathbf{k}_{3}, \mathbf{k}_{4}}^{\alpha_{1}, \alpha_{2}, \alpha_{4}}$ is the four-fermion interaction, $\psi_{\mathbf{k}, \alpha}^{\dagger}$ is the creation operator for fermions with spin $\alpha$ and momentum $\mathbf{k}$, and $\varepsilon_{\mathbf{k}}$ is the band-structure dispersion. For a one band Hubbard model with local Coulomb interaction,

$$
\begin{aligned}
U_{\mathbf{k}_{1}, \mathbf{k}_{2}, \mathbf{k}_{3}, \mathbf{k}_{4}}^{\alpha_{1}, \alpha_{2}, \alpha_{3}, \alpha_{4}}= & U \delta_{\mathbf{k}_{\mathbf{1}}+\mathbf{k}_{\mathbf{2}}-\mathbf{k}_{\mathbf{3}}-\mathbf{k}_{\mathbf{4}}} \\
& \times\left(\delta_{\alpha_{1} \alpha_{4}} \delta_{\alpha_{2} \alpha_{3}}-\delta_{\alpha_{1} \alpha_{3}} \delta_{\alpha_{2} \alpha_{4}}\right) .
\end{aligned}
$$

In a perturbation theory for Eq. 17 involving $U$ and the fermion band width, the contributions from large and small fermionic energies are mixed. However, near a magnetic instability much of the non-trivial physics is associated (at any $U$ ) with the system behavior at low energies. To single out this low-energy sector, one can borrow a strategy from field theory: introduce a characteristic energy cut off, $\Lambda$, and generate an effective low energy model by eliminating all degrees of freedom above $\Lambda$ in the hope that some of the system properties will be universally determined by the low-energy sector and as such will not depend sensitively on the actual choice of $\Lambda$. Eliminating these high energy degrees of freedom is the central theoretical difficulty in the field of strongly correlated electron systems. In our case, except for a poorly controlled RPA analysis, there are no known ways to perform such a renormalization procedure. Furthermore, the separation between high energies and low-energies can be rigorously justified only if the interaction is smaller than the fermionic bandwidth. For larger interactions, the critical exponents very likely will remain the same, but the pre-factors will depend on system properties far from the Fermi surface.

Still, it is possible to assume that the separation of scales is possible, i.e., that the interaction is smaller than the bandwidth and to pursue the consequences of that assumption. This is the strategy we adopt. By itself, this does not guarantee that there exists a universal physics confined to low energies. This we will have to prove. This also does not mean that the system is in the weak coupling regime, as near the antiferromagnetic transition we will find a strong, near-divergent contribution to the fermionic self-energy that comes from low frequencies. What the separation of scales actually implies (to the extent that we find universal, low-energy physics) is that Mott physics does not play a major role. In particular, in our analysis the Fermi surface in the normal state remains large, and its volume satisfies Luttinger theorem. How well this approximation is satisfied depends on doping for a given material and also varies from one material to another. Most of our experimental comparisons will be made with the cuprates. In cuprates, the Hubbard $U$ in the effective one-band model for $\mathrm{CuO}_{2}$ unit (a charge transfer gap) is estimated to be between 1 and 2 $\mathrm{eV}$. The bandwidth, measured by ARPES and resonant Raman experiments, roughly has the same value. This suggests that lattice effects, beyond a universal low energy theory, do, indeed, play some role. At half-filling, lattice effects are crucial as evidenced by the fact that half-filled materials are both Mott insulators and antiferromagnets with local (nearest-neighbor) spin correlations. Doping a Mott insulator almost certainly initially produces a small Fermi surface (hole or electron pockets). This small Fermi surface evolves as doping increases and eventually transforms into a large, "Luttinger" Fermi surface. How this evolution actually occurs is still a subject of debate. From our perspective, it is essential that at and above optimal doping, all ARPES data indicate that the Fermi surface is large. Correspondingly, magnetooscillation experiments in BEDT-TTF based organic superconductors also show that the Fermi surface of these materials is large. We believe that in this situation, lattice effects change the system behavior quantitatively but not qualitatively, and the neglect of lattice effects is justified. We emphasize however that our analysis certainly needs to be modified to incorporate Mott physics close to half-filling.

Several aspects of our approach have a close similarity to the fluctuation exchange approximation (FLEX), which in case of a single band Hubbard model corresponds to a self consistent summation of bubble and 
ladder diagrams 103 104. Specifically, the emergence of a sharp gapless resonance mode in the spin excitation spectrum of a d-wave superconductor, the feedback of this mode on the fermions and the anomalous normal state behavior of low energy fermions close to an antiferromagnetic instability are very similar in both approaches105 108. On the other hand, the FLEX approach attempts to determine the static spin response and thus the actual position of the quantum critical point in terms of the bare parameters of the model such as the local or additional nonlocal Coulomb repulsions as well as the band structure $\varepsilon_{\mathbf{k}}$. As discussed above, the static spin response, characterized by the correlation length $\xi$, strongly depends on the behavior of fermions with large energy. Details of the underlying microscopic model which are hard to specify uniquely as well as uncontrolled approximations in the treatment of the high energy behavior strongly affect the static spin response within the FLEX approach, making it hard to discriminate model dependent aspects from universal behavior. It is this latter aspect which is resolved in our approach which concentrates exclusively on the universal low energy physics for a given $\xi$.

What should be the form of the low-energy action? Clearly, it should involve fermions which live near the Fermi surface. It also should involve collective spin bosonic degrees of freedom with momenta near $\mathbf{Q}$, as these excitations become gapless at the magnetic transition. The most straightforward way to obtain this action is to introduce a spin-1 bose field $\mathbf{S}$ and decouple the four-fermion interaction using the Hubbard-Stratonovich procedure116.117. This yields

$$
\begin{array}{r}
\mathcal{H}=\sum_{\mathbf{k}, \alpha} \varepsilon_{\mathbf{k}} \psi_{\mathbf{k}, \alpha}^{\dagger} \psi_{\mathbf{k}, \alpha}+\sum_{\mathbf{q}} U(\mathbf{q}) \mathbf{S}_{\mathbf{q}} \cdot \mathbf{S}_{-\mathbf{q}} \\
+\sum_{\mathbf{k}, \mathbf{q}, \alpha, \beta} U(\mathbf{q}) \psi_{\mathbf{k}+\mathbf{q}, \alpha}^{\dagger} \sigma_{\alpha \beta} \psi_{\mathbf{k}, \beta} \cdot \mathbf{S}_{-\mathbf{q}}
\end{array}
$$

where the $\sigma_{\alpha \beta}$ are Pauli matrices and we assumed that the four fermion interaction only makes a contribution in the spin channel with momentum transfer q. Integrating formally over energies larger than $\Lambda$ we obtain the effective action in the form (see e.g., 118)

$$
\begin{aligned}
\mathcal{S}= & -\int_{k}^{\Lambda} G_{0}^{-1}(k) \psi_{k, \alpha}^{\dagger} \psi_{k, \alpha}+\frac{1}{2} \int_{q}^{\Lambda} \chi_{0}^{-1}(q) \mathbf{S}_{q} \cdot \mathbf{S}_{-q} \\
& +g \int_{k, q}^{\Lambda} \psi_{k+q, \alpha}^{\dagger} \sigma_{\alpha \beta} \psi_{k, \beta} \cdot \mathbf{S}_{-q}+O\left(S^{4}\right)
\end{aligned}
$$

The last term is a symbolic notation for all terms with higher powers of $S$. Fortunately, in dimensions $d \geq 2$ these higher order terms are irreleyent (marginal for $d=2$ ) and can therefore be neglected 77.78 .

The integration over $k$ and $q$ in 4 is over $2+1$ dimensional vectors $q=\left(\mathbf{q}, i \omega_{m}\right)$ with Matsubara frequency $\omega_{m}$. In explicit form, the integrals read

$$
\int_{q}^{\Lambda} \ldots=\int_{|\mathbf{q}-\mathbf{Q}|<\Lambda} \frac{d^{d} \mathbf{q}}{(2 \pi)^{d}} T \sum_{m} \ldots
$$

in the boson case, and

$$
\int_{k}^{\Lambda} \ldots=\int_{\left|\mathbf{k}-\mathbf{k}_{F}\right|<\Lambda} \frac{d^{d} \mathbf{k}}{(2 \pi)^{d}} T \sum_{m} \ldots
$$

in the fermion case. Further, $g$ is the effective coupling constant, $G_{0}(k)$ is the bare low-energy fermion propagator, and $\chi_{0}(q)$ is the bare low-energy collective spin boson propagator. As we have emphasized, a controlled derivation of $g, G_{0}(k)$, and $\chi_{0}(q)$ is impossible. We therefore will not try to calculate $g$, etc. Rather, we use the fact that antiferromagnetism predominantly comes from high-energy fermions and further assume that the integration over high energies does not produce singularities in both bosonic and fermionic propagators. Then, quite generally, $G_{0}(k)$, and $\chi_{0}(q)$ should have Fermi-liquid and Ornstein-Zernike forms, respectively

$$
\begin{gathered}
G_{0}(k)=\frac{z_{0}}{i \omega_{m}-\epsilon_{\mathbf{k}}}, \\
\chi_{0}(q)=\frac{\alpha}{\xi_{0}^{-2}+(\mathbf{q}-\mathbf{Q})^{2}+\omega_{m}^{2} / c^{2}} .
\end{gathered}
$$

Here, $\xi_{0}$ is the bare value of the spin correlation length, and the other notations are self-explanatory. The actual $\xi$ generally differs from the bare one because the low energy fermions that damp the spin fluctuation modes might change as well their static properties. $\xi$ acquires an additional temperature dependence due to spin-spin interactions. We return to this question later. It is essential that the bare spin propagator does not contain a term linear in $\omega$. The latter will only appear when we consider the interaction within the low-energy model.

We also assume that (i) the momentum dependence of the effective coupling $g$ is non-singular and can be neglected (recall that we are only interested in a narrow range of bosonic momenta near $\mathbf{Q}$ ), (ii) the lowenergy fermionic dispersion can be linearized in $\mathbf{k}-\mathbf{k}_{F}$ : $\epsilon_{\mathbf{k}}=\mathbf{v}_{F} \cdot\left(\mathbf{k}-\mathbf{k}_{F}\right)$, and (iii) that the Fermi velocity is non-singular near hot spots and to first approximation its magnitude can be approximated by a constant. In doing this we neglect effects due to a van Hove singularity in the density of states. Finally, the exact values of $z_{0}$ and $\alpha$ are not relevant, as both can be absorbed into an effective coupling with dimension of an energy

$$
\bar{g}=g^{2} z_{0}^{2} \alpha
$$

while $v_{F}$ and $\xi$ will always appear only in the combination $v_{F} \xi^{-1}$.

We see therefore that the input parameters in Eq. A are the effective coupling energy $\bar{g}$, the typical quasiparticle energy $v_{F} \xi^{-1}$, and the upper cutoff $\Lambda$. An additional parameter is the angle $\phi_{0}$ between the Fermi velocities at 
the two hot spots separated by $\mathbf{Q}$, but this angle does not enter the theory in any significant manner as long as it is different from 0 or $\pi$. When hot spots are located near $(0, \pi)$ and $(\pi, 0)$ points, as in optimally doped cuprates, $\phi_{0}$ is close to $\pi / 2$, the value we use in what follows.

As we have emphasized, we will demonstrate that the low-energy properties of the model are universal and do not depend on $\Lambda$, which then can be set to infinity. Out of the two parameters that are left, one can construct a doping dependent dimensionless ratio

$$
\lambda=\frac{3}{4 \pi} \frac{\bar{g}}{v_{\mathrm{F}} \xi^{-1}}
$$

which will turn out to be the effective dimensionless coupling constant of the problem (the factor $3 / 4 \pi$ is introduced for further convenience). The fact that $\lambda$ scales with $\xi$ immediately implies that close enough to a magnetic transition $\lambda>1$, i.e., the system will necessarily be in a strong coupling limit. Besides $\lambda$ the only other free parameter of the theory is an overall energy scale, i.e., $\bar{g}$ (or alternatively the quasiparticle energy $v_{\mathrm{F}} \xi^{-1}$ ). All physical quantities that we discuss will be expressed in terms of these two parameters only.

Eqs. 48 determine the structure of the perturbation theory of the model. The interaction between fermions and collective spin excitations yields self energy corrections to both bosonic and fermionic propagators. We will show below that at strong coupling, the fermionic selfenergy strongly depends on frequency and also displays some dependence on the momentum along the Fermi surface. However, its dependence on the momentum transverse to the Fermi surface can be neglected together with vertex corrections. The fermionic and bosonic propagators are then given by the Gor'kov expressions, which for generality we present in the superconducting state,

$$
\begin{gathered}
G_{\mathbf{k}}(i \omega)=\frac{i \omega+\Sigma_{\mathbf{k}}(i \omega)+\varepsilon_{\mathbf{k}}}{\left(\left(i \omega+\Sigma_{\mathbf{k}}(i \omega)\right)^{2}-\Phi_{\mathbf{k}}^{2}(i \omega)-\varepsilon_{\mathbf{k}}^{2}\right.}, \\
F_{\mathbf{k}}(i \omega)=-\frac{\Phi_{\mathbf{k}}(i \omega)}{\left(i \omega+\Sigma_{\mathbf{k}}(i \omega)\right)^{2}-\Phi_{\mathbf{k}}^{2}(i \omega)-\varepsilon_{\mathbf{k}}^{2}} \\
\chi_{\mathbf{q}}(i \omega)=\frac{\alpha \xi^{2}}{1+\xi^{2}(\mathbf{q}-\mathbf{Q})^{2}-\Pi_{\mathbf{Q}}(i \omega)} .
\end{gathered}
$$

Here $\Sigma_{\mathbf{k}}(i \omega)$ and $\Pi_{\mathbf{Q}}(i \omega)$ are fermionic and bosonic selfenergies (k stands for the component along the Fermi surface), and $F_{\mathbf{k}}(i \omega)$ and $\Phi_{\mathbf{k}}(i \omega)$ are the anomalous Green's function and the anomalous self-energy, respectively. In the next sections we compute $\Sigma_{\mathbf{k}}(i \omega), \Pi_{\mathbf{Q}}(i \omega)$ and $\Phi_{\mathbf{k}}(i \omega)$.

One can also motivate the spin fermion model by following the appraach developed by Landau in his theory of Fermi liquids 9 , i.e., by assuming that the influence of the other fermionic quasiparticles on a given quasiparticle can be described in terms of a set of molecular fields 123. In the present case the dominant molecular field is an exchange field produced by the Coulomb interaction $U(\mathbf{q})$. However, one has to consider this field as dynamic, not static. The corresponding part of the action contains

$$
\mathcal{S}=-\int_{k}^{\Lambda} G_{0}^{-1}(k) \psi_{k, \alpha}^{\dagger} \psi_{k, \alpha}+\frac{1}{2} \int_{q}^{\Lambda} \mathbf{H}_{q}^{\mathrm{int}} \cdot \mathbf{s}_{-q}
$$

with fermionic spin density $\mathbf{s}_{-q}=\int_{k}^{\Lambda} \psi_{k+q, \alpha}^{\dagger} \sigma_{\alpha \beta} \psi_{k, \beta}$. As in the Landau theory of Fermi liquids, we assume that the molecular field $\mathbf{H}_{q}^{\text {int }}$ is given by the linear response function,

$$
\mathbf{H}_{q}^{\mathrm{int}}=g^{2} \chi_{0}(q) \mathbf{s}_{-q}
$$

This expression is valid as long as one is not in a region so close to a magnetic instability that nonlinear magnetic effects play an important role. Formally, this expression can be obtained from Eq. 4 by integrating out the collective degrees of freedom $\mathbf{S}$. A relation between this purely fermionic approach and the bosonic spin susceptibility $\chi(\mathbf{q})$ of Eq. 13 can be established by evaluating the reducible four point vertex in the spin channel in the lowest order of perturbation theory. We find

$$
\Gamma_{\alpha \beta, \gamma \delta}\left(k, k^{\prime}, q\right)=-V_{\mathrm{eff}}(q) \sigma_{\alpha \beta} \sigma_{\gamma \delta}
$$

where the effective quasiparticle interaction is proportional to the renormalized spin propagator of Eq. 13 .

$$
V_{\mathrm{eff}}(q)=g^{2} \chi(q)=\frac{g^{2} \alpha \xi^{2}}{1+\xi^{2}(\mathbf{q}-\mathbf{Q})^{2}-\Pi_{\mathbf{Q}}(\omega)} .
$$

\section{B. Weak coupling approach to the pairing instability}

One of the most appealing aspects of the spinfluctuation theory is that it inevitably yields an attraction in the $d_{x^{2}-y^{2}}$ channel. As with any unconventional pairing, this attraction is the result of a specific momentum dependence of the interaction, not of its overall sign which for spin fluctuation exchange is positive, i.e., repulsive in the $s$-wave channel. This differentiates spin induced pairing from the pairing mediated by phonons. In the latter case, the effective interaction between fermions is negative up to a Debye frequency. In configuration space the spin-fluctuation interaction, Eq.17, can easily seen to be repulsive at the origin and alternate between attraction and repulsion as one goes to nearest neighbors. It is always repulsive along the diagonals, and this is why a $d_{x^{2}-y^{2}}$ state, in which the nodes of the gap are along the diagonals, is the energetically preferred pairing state due to spin-fluctuation exchange 110 .

Suppose first that the spin-fermion coupling is small enough such that conventional perturbation theory is valid. To second order in the spin-fermion coupling, the spin mediated interaction has the following form, Eq. 16. 
The total antisymmetry of the interaction implies that the orbital part is symmetric when the spin part is antisymmetric and vice versa. The orbital part of the interaction at low frequencies has the same sign as the propagator of optical phonons at $\omega<\omega_{D}$. However, the spin part involves a convolution of the Pauli matrices, and is different from phononic $\delta_{\alpha \beta} \delta_{\gamma \delta}-\delta_{\alpha \delta} \delta_{\beta \gamma}$. Using $\sigma_{\alpha \beta} \sigma_{\gamma \delta}=2 \delta_{\alpha \delta} \delta_{\beta \gamma}-\delta_{\alpha \beta} \delta_{\gamma \delta}$ we find

$$
\Gamma_{\alpha \gamma, \beta \delta}(q)=-g^{2} \chi(q)\left(T_{\alpha \gamma, \beta \delta}-3 S_{\alpha \gamma, \beta \delta}\right)
$$

where $T_{\alpha \gamma, \beta \delta}=\left(\delta_{\alpha \beta} \delta_{\gamma \delta}+\delta_{\alpha \delta} \delta_{\beta \gamma}\right) / 2$ and $S_{\alpha \gamma, \beta \delta}=$ $\left(\delta_{\alpha \beta} \delta_{\gamma \delta}-\delta_{\alpha \delta} \delta_{\beta \gamma}\right) / 2$ are triplet and singlet spin configurations, respectively. We see that there is an extra minus sign in the singlet channel. This obviously implies that in distinction to phonons, the isotropic $s$-wave component of the interaction is repulsive, i.e., isotropic $s$-wave pairing due to spin fluctuation exchange is impossible. There are two other possibilities: unconventional singlet pairing for which we will need a partial component of $\chi(q)$ to be negative, or triplet pairing, for which the corresponding partial component of $\chi(q)$ should be positive.

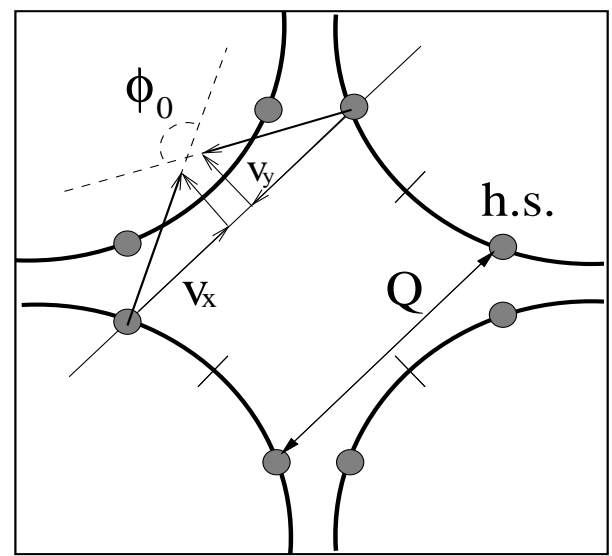

FIG. 6. A representative Brillouin zone and Fermi surface for a near-optimally doped cuprate superconductor. The hot spots are connected by the antiferromagnetic wave vector $\mathbf{Q}=(\pi, \pi)$. For a weakly incommensurate magnetic response, the number of hot spots doubles but simultaneously the intensity of the magnetic response at momenta connecting pairs of hot spots drops by a factor of two. The net effect then remains the same as for the commensurate response. The figure is taken from 51 .

It turns out that for a spin susceptibility peaked at or near the antiferromagnetic momentum $\mathbf{Q}$, the triplet components are all negative, and triplet pairing is impossible. We therefore focus on singlet pairing. The linearized equation for the pairing vertex $F_{\mathbf{k}}\left(i \omega_{m}\right)$ in the singlet channel is

$$
F(k)=-3 g \int_{k^{\prime}}^{\Lambda} F\left(k^{\prime}\right) \chi\left(k-k^{\prime}\right) G_{0}\left(k^{\prime}\right) G_{0}\left(-k^{\prime}\right) .
$$

If $\chi\left(k-k^{\prime}\right)=\chi_{\mathbf{k}-\mathbf{k}^{\prime}}\left(i \omega_{m}-i \omega_{m}^{\prime}\right)$ was independent of momentum, a solution of this equation would not exist. In our situation, however, the interaction predominantly occurs between fermions that are separated in momentum space by $\mathbf{Q}=(\pi, \pi)$ (see Fig.6).

Since both $\mathbf{k}$ and $\mathbf{k}+\mathbf{Q}$ should be near the Fermi surface, the pairing predominantly involves fermions near hot spots $\mathbf{k}_{\mathrm{hs}}$ and $\mathbf{k}_{\mathrm{hs}}+\mathbf{Q}$. As the hot spots are well separated in momentum space, we can change the sign in the r.h.s. of Eq.19 by requiring that the pairing gap changes sign between $\mathbf{k}_{\mathrm{hs}}$ and $\mathbf{k}_{\mathrm{hs}}+\mathbf{Q}$. Indeed, substituting $F_{\mathbf{k}+\mathbf{Q}}\left(i \omega_{m}\right)=-F_{\mathbf{k}}\left(i \omega_{m}\right)$ into Eq.19, we find that both sides of this equation have the same sign. The pairing problem then becomes almost identical to that for phonons, and the gap equation has a solution at some $T_{c}$.

Altogether, there are $N=8$ hot spots in the Brillouin zone; these form 4 pairs separated by $\mathbf{Q}$, between which the gap should change sign. This still does not specify the relative sign of the gap between adjacent hot spots. However, if the hot spots are close to $(0, \pi)$ and symmetry related points, as in optimally doped cuprates, the gap is unlikely to change sign between them. This leaves as the only possibility a gap that vanishes along diagonal directions $k_{x}= \pm k_{y}$, i.e., it obeys $d_{x^{2}-y^{2}}$ symmetry.

We emphasize that although we found that the pairing gap necessarily should have $d_{x^{2}-y^{2}}$ symmetry, its momentum dependence is generally more complex than simply $\cos k_{x}-\cos k_{y}$. To understand this, we consider how one can generally analyze Eq.1922. A standard strategy is to expand both the pairing vertex and the interaction in the eigenfunctions of the representations of the $D_{4 h}$ symmetry group of the square lattice. There are four singlet representations of $D_{4 h}$ labeled $A_{1 g}, B_{1 g}, B_{2 g}$ and $A_{2 g}$. The basic eigenfunctions in each representation are 1 in $A_{1 g}, \cos k_{x}-\cos k_{y}$ in $B_{1 g}, \sin k_{x} \sin k_{y}$ in $B_{2 g}$ and $\left(\cos k_{x}-\cos k_{y}\right) \sin k_{x} \sin k_{y}$ in $A_{2 g}$. Other eigenfunctions in each representation are obtained by combining the basic eigenfunction with the full set of eigenfunctions with full $D_{4 h}$ symmetry. Obviously, the eigenfunctions with $d_{x^{2}-y^{2}}$ symmetry belong to $B_{1 g}$ channel. The orthogonal functions in the set can be chosen as $d_{n}(\mathbf{k})=\cos n k_{x}-\cos n k_{y}$.

One can easily make sure that the pairing problem close to $\mathrm{T}_{c}$ decouples between different representations. As $\xi \rightarrow \infty$, the $B_{1 g}$ components of the spin susceptibility diverge while other components remain finite. Obviously, the pairing is in the $B_{1 g}$ channel. At moderate $\xi$, all components are generally of the same order, but numerical calculations show that $B_{1 g}$ components continue to be the largest. We therefore neglect other channels and focus only on $B_{1 g}$ pairing. Still, there are an infinite number of $B_{1 g}$ eigenfunctions, and the pairing problem does not decouple between them. Indeed, expanding $F_{\mathbf{k}}\left(\omega_{m}\right)$ and $\chi\left(\mathbf{k}, \omega_{m}\right)$ in $d_{n}(\mathbf{k})$ as $F_{\mathbf{k}}=\sum_{p} F_{p} d_{p}(\mathbf{k})$, $\chi\left(\mathbf{k}-\mathbf{k}^{\prime}, i \omega-i \omega^{\prime}\right)=\sum_{p} \chi_{p}\left(i \omega-i \omega^{\prime}\right) d_{p}(\mathbf{k}) d_{p}\left(\mathbf{k}^{\prime}\right)$ and substituting the result into 19 we obtain 122

$$
F_{n}\left(i \omega_{m}\right)=-3 g^{2} T \sum_{p, m^{\prime}} F_{p}\left(i \omega_{m^{\prime}}\right) \chi_{n}\left(i \omega_{m-m^{\prime}}\right) \Gamma_{n, p}
$$


where

$$
\Gamma_{n, p}=\int \frac{d^{2} k^{\prime}}{4 \pi^{2}} \frac{d_{n}\left(\mathbf{k}^{\prime}\right) d_{p}\left(\mathbf{k}^{\prime}\right)}{\left(\omega_{m}^{\prime}\right)^{2}+\left(\epsilon_{\mathbf{k}}^{\prime}\right)^{2}}
$$

We see that all $\Gamma_{n, p}$ are nonzero for arbitrary $n$ and $p$ as the products $d_{n}(k) d_{p}(k)$ and $G_{0}(k) G_{0}(-k)=1 /\left(\left(\omega_{m}\right)^{2}+\right.$ $\left.\left(\epsilon_{\mathbf{k}}\right)^{2}\right)$ are symmetric under $D_{4 h}$. As a result, Eq. 20 couples together an infinite number of partial components $F_{n}$. As long as the partial components of the susceptibility are dominated by momenta relatively far from $\mathbf{Q}$, all $\chi_{n}$ and hence $F_{n}$ are of the same order. In some particular lattice models, e.g. in the Hubbard model with nearest neighbor interaction, $U=4 t$ and $\xi \sim 2$, an RPA analysis shows that $\chi_{1}$ is numerically significantly larger than all other $\chi_{n} 37$. In this situation, $F_{\mathbf{k}} \approx F_{1} d_{1}(\mathbf{k})$, i.e., the momentum dependence of the gap should closely resemble the $\cos k_{x}-\cos k_{y}$ form. In real space, this implies that the pairing (even though non-local in time) is local and involves fermions from the nearest neighbor sites on the lattice. In models with different high-energy physics, other $\chi_{n}$ may dominate. Clearly, the momentum dependence of the gap is not universal as long as the pairing is local in real space.

Universality is recovered when the susceptibility becomes strongly peaked at $\mathbf{Q}$. In this limit (in which we can also rigoropsly justify restricting our attention to the $B_{1 g}$ channel 22 ), all partial components $\chi_{n}$ scale as $\log \xi$ and differ only in sub-leading non-logarithmic terms. A straightforward trigonometric exercise shows that for near-equal $\chi_{n}$, the pairing gap is very different from $\cos k_{x}-\cos k_{y}$ : it is reduced near the nodes and enhanced near the maxima at hot spots with the ratio of the gap maximum and the slope near the nodes increasing as $\xi^{2}$. In this situation the pairing problem is confined to hot spots, and the pairing symmetry is inevitably $d_{x^{2}-y^{2}}$.

\section{SUMMARY OF STRONG COUPLING THEORY FOR ELECTRON PHONON PAIRING}

For phonon-mediated superconductivity, Eliashberg theory offers a successful approach to study the system behavior at strong coupling. It is justified by Migdal's theorem which states that the vertex corrections, $\delta g / g$ and $\left(1 / v_{F}\right) d \Sigma(\mathbf{k}, i \omega) / d k$, are small due to the smallness of the ratio of sound velocity and Fermi velocity stemming from the smallness of the ratio of the electronic and ionic masses. Eliashberg has demonstrated that for $\Sigma(\mathbf{k}, i \omega) \approx \Sigma(i \omega)$ and $g_{\mathrm{tot}}=g+\delta g \simeq g$, the phononmediated pairing problem can be solved exactly. A review of Eliashberg theory for the electron-phonon problem is given in Ref. 119. As we already pointed out, for spin fluctuation induced pairing there is no Migdal theorem simply because the spin propagator is made out of electrons and hence a typical spin velocity is of the same order as the Fermi velocity. It is therefore natural to inquire whether this implies that an Eliashberg-type treatment is inapplicable to the spin problem. To properly address this issue, we review the case of electron- phonon interactions and examine why the smallness of the mass ratio affects $d \Sigma(\mathbf{k}, i \omega) / d \mathbf{k}$ but not $d \Sigma(\mathbf{k}, i \omega) / d \omega$.

The electron-phonon interaction is generally rather ip volved and has been discussed in detail in the literature For our purposes, it is sufficient to consider the simplest Fröhlich-type model of the electron-phonon interaction in which low-energy electrons are coupled to optical phonons by a momentum, frequency and polarization independent interaction $g_{\mathrm{ep}}$. Despite its simplicity, this model captures the key physics of phonon-mediated pairing.

The propagator of an optical phonon has the form

$$
D\left(\mathbf{q}, i \omega_{m}\right)=\frac{\omega_{0}}{\omega_{0}^{2}+\omega_{m}^{2}+\left(v_{s} \mathbf{q}\right)^{2}}
$$

Here $\omega_{0}$ is a typical phonon frequency, which is of the order of the Debye frequency, and $v_{s}$ is the sound velocity. Both $\omega_{0}$ and $v_{s}$ are input parameters, unrelated to fermions. The ratio $v_{s} / v_{F}$ scales as $(m / M)^{1 / 2}$ where $M$ is the ionic mass, and $m$ is the electron mass. In practice, $v_{s} / v_{F} \sim 10^{-2}$. 5 . This is a real physically motivated small parameter for the electron- phonon problem.

Far from structural instabilities, $\omega_{0} \sim v_{s} a$ where a is the distance between ions. To make the analogy with spin fluctuations more transparent, we will consider below the case when $\omega_{0} \ll v_{s} a$, i.e., the correlation length for optical phonons is large. This last assumption allows us to simplify the calculations but will not change their conclusions.

In the presence of two different velocities, it is not $a$ priori obvious what is the best choice of a dimensionless expansion parameter in the theory. Two candidate dimensionless parameters are

$$
\lambda_{\mathrm{ep}}=g_{\mathrm{ep}}^{2} /\left(4 \pi\left(v_{s} v_{F}\right)\right)
$$

(the factor $4 \pi$ is chosen for further convenience), and

$$
\tilde{\lambda}_{\mathrm{ep}}=\lambda_{\mathrm{ep}} v_{s} / v_{F} \sim\left(g_{\mathrm{ep}} / v_{F}\right)^{2} .
$$

For simplicity, we set the lattice constant $a=1$. Obviously, $\lambda_{\text {ep }} \gg \tilde{\lambda}_{\text {ep }}$. In practice, $\tilde{\lambda}_{\text {ep }} \ll 1$ for all reasonable $g_{\text {ep }}$ while $\lambda_{\text {ep }}$ can be either small or large.

Which of the two dimensionless couplings appears in perturbation theory? Consider first the lowest-order diagram for the spin-fermion vertex at zero external frequency and zero bosonic momentum with all fermionic momenta at the Fermi surface. Choosing $T=0$ and the $x$ axis along the direction of $\mathbf{v}_{F}$ of an external fermion, we obtain the vertex correction in the form:

$$
\frac{\delta g_{\mathrm{ep}}}{g_{\mathrm{ep}}}=\frac{g_{\mathrm{ep}}^{2}}{8 \pi^{3}} \int \frac{d \omega_{m} d^{2} q}{\left(i \omega_{m}-v_{F} q_{x}\right)^{2}} \frac{\omega_{0}}{\omega_{0}^{2}+\omega_{m}^{2}+\left(v_{s} \mathbf{q}\right)^{2}} .
$$

The evaluation of the momentum and frequency integrals is straightforward. On performing the integration we obtain for $\omega_{0} \ll v_{F}$, 


$$
\frac{\delta g_{\mathrm{ep}}}{g_{\mathrm{ep}}}=\tilde{\lambda}_{\mathrm{ep}}=\lambda_{\mathrm{ep}} \frac{v_{s}}{v_{F}} .
$$

We see that the vertex correction scales as $\tilde{\lambda}_{\text {ep }}$ and is small. For $\omega_{0} \sim v_{s}$, the expression is more complex but still is of order $\tilde{\lambda}$. One can easily check that higher-order diagrams yield higher powers of $\tilde{\lambda}_{e p}$ i. ., are also small. This result, first obtained by Migdal125, is Migdal's theorem.

The physics behind this result is transparent and can be directly deduced from an analysis of the integrand in Eq.25. We see that the product of the two fermionic Green's functions yields a double pole at $q_{x}=i \omega_{m} / v_{F} \sim$ $\omega_{0} / v_{F}$. The integral over $q_{x}$ is then finite only due to the presence of the pole in the bosonic propagator which is located at a much larger momentum $q_{x} \sim i \omega_{0} / v_{s}$. This implies that the electrons that contribute to the vertex correction are oscillating not at their own fast frequencies but at much slower phonon frequencies. At vanishing $v_{s} / v_{F}$, fermions can be considered as static objects with a typical $q_{x} \sim q_{y} \sim \omega_{0} / v_{s}$. The two dimensional momentum integration over the product of two fermionic propagators then yields $O\left(1 / v_{F}^{2}\right)$. Simultaneously, the frequency integral over the bosonic propagator yields $D\left(q \sim \omega_{0} / v_{s}, 0\right)=O(1)$. Combining the two results, we obtain $\delta g_{\mathrm{ep}} / g_{\mathrm{ep}}=O(\tilde{\lambda})$ as in Eq. 26.

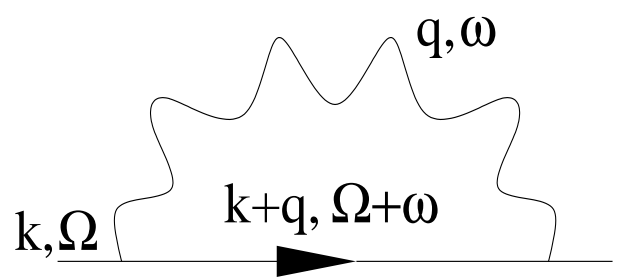

FIG. 7. A Feynman diagram for the electronic self energy due to electron phonon interaction in the Eliashberg theory.

We next consider the fermionic self-energy. Evaluating the diagram in Fig.7 and subtracting the contribution at $\omega=0, \mathbf{k}=\mathbf{k}_{F}$, we obtain

$$
\Sigma\left(\mathbf{k}, i \omega_{m}\right)=\left(i \omega_{m}-\epsilon_{\mathbf{k}}\right) I\left(\mathbf{k}, i \omega_{m}\right)
$$

where

$$
\begin{aligned}
I\left(\mathbf{k}, i \omega_{m}\right)= & g_{\mathrm{ep}}^{2} \int \frac{d \Omega_{m} d^{2} q}{8 \pi^{3}} \frac{\omega_{0}}{\omega_{0}^{2}+\Omega_{m}^{2}+\left(v_{s} \mathbf{q}\right)^{2}} \\
& \times \frac{1}{i \Omega_{m}-v_{F} q_{x}} \frac{1}{i\left(\omega_{m}+\Omega_{m}\right)-\epsilon_{k}-v_{F} q_{x}}
\end{aligned}
$$

We again have chosen the $q_{x}$ axis along the Fermi velocity $\mathbf{v}_{F}$.

Suppose for a moment that $I\left(\mathbf{k}, i \omega_{m}\right)$ is non-singular in the limit $\mathbf{k} \rightarrow 0$ and $\omega_{m} \rightarrow 0$. Then the wave function renormalization and the velocity renormalization are both expressed via $I(0,0)$. This quantity can be evaluated in exactly the same way as the vertex correction. Performing the integration, we find

$$
I(0,0)=-\tilde{\lambda}_{\text {ep }}
$$

Substituting this into Eq.27 we find $v_{F}^{-1} d \Sigma / d k=$ $-d \Sigma / d(i \omega)=\tilde{\lambda}_{\text {ep }}$. As $\tilde{\lambda}_{\text {ep }} \ll 1$, this result, if true, would imply that both derivatives of the self-energy are small, i.e., the system is fully in the weak coupling regime. The physical interpretation of this result would parallel that for the vertex correction: fast electrons vibrate at slow phonon velocities, and this well-out-of-resonance vibration cannot substantially affect electronic properties.

However, this result is incorrect $-d \Sigma / d \omega$ is of order $\lambda_{\text {ep }}$, not $\tilde{\lambda}_{e p}$. The answer lies in the singular behavior of $I\left(\mathbf{k}, i \omega_{m}\right)$ in the limit of vanishing $\mathbf{k}$ and $\omega_{m}$. To see this, we need to compute $I(\mathbf{k}, i \omega)$ in Eq 28 at small but finite $\omega$ and $\epsilon_{\mathbf{k}}$. An explicit computation yields

$$
I\left(\mathbf{k}, i \omega_{m}\right)=-\tilde{\lambda}_{e p}+\lambda_{e p} \frac{i \omega_{m}}{i \omega_{m}-\epsilon_{\mathbf{k}}}
$$

demonstrating that $I(0,0)$ and the limit of $I(\mathbf{k}, i \omega)$ at $\omega, \epsilon_{\mathbf{k}} \rightarrow 0$ do not coincide. The additional contribution in Eq. 30 comes from the regularization of the double pole in the integrand in Eq.28 and is in fact mathematically similar to the chiral anomaly in quantum chromodynamics 126.

It is clear from Eq. 30 that $I\left(\mathbf{k}, i \omega_{m}\right)$ has singular low frequency and small momentum limits. Substituting Eq.30 into Eq.27, we find that the actual self-energy is

$$
\Sigma\left(\mathbf{k}, i \omega_{m}\right)=i \lambda_{e p} \omega_{m}-\tilde{\lambda}_{e p}\left(i \omega_{m}-\epsilon_{\mathbf{k}}\right) .
$$

We see that $d \Sigma / d \omega$ scales with $\lambda_{e p}$ whereas $v_{F}^{-1} d \Sigma / d k$ is proportional to $\tilde{\lambda}_{\mathrm{ep}}$. At strong coupling, $\lambda_{e p} \geq 1$, this self-energy gives rise to a strong renormalization of both the quasiparticle mass and the quasiparticle wave function. Still, vertex corrections $\underset{\sim}{\text { and }}$ the renormalization of the Fermi velocity scale with $\tilde{\lambda}_{e p}$ and are small

To understand the physical origin of the distinction between the frequency and momentum dependence of the fermionic self-energy, it is essential to realize that the second, $O\left(\lambda_{e p}\right)$, term in Eq. 30 is not caused by real electron-phonon scattering. Rather, a careful examination of the structure of the expression for $I\left(\mathbf{k}, \omega_{m}\right)$ shows that this term accounts for the pole in the fermionic particle hole polarization bubble at small momentum and frequency. This pole is known to describe a zero sound bosonic collective excitation - a vibration of the Fermi surface in wich it changes its form but preserves its volume.133 134 This implies that the fermionic self-energy is actually caused by coupling of fermions to their own zero-sound collective modes, while phonons just mediate this coupling.

The neglect of vertex corrections and $v_{F}^{-1} d \Sigma / d k$ leads to the well known Eliashberg equations for $\Sigma_{m}=\Sigma\left(i \omega_{m}\right)$ and (in the superconducting state) the pairing self energy $\Phi_{m}=\Phi\left(i \omega_{m}\right)$ that also depends only on frequency. For phonon-mediated superconductors, these equations have the form 


$$
\begin{aligned}
& \Sigma_{m}=\frac{\lambda_{e p} T}{2} \sum_{n} \frac{\left(\omega_{n}+\Sigma_{n}\right) D\left(i \omega_{m-n}\right)}{\sqrt{\Phi_{n}^{2}+\left(\omega_{n}+\Sigma_{n}\right)^{2}}} \\
& \Phi_{m}=\frac{\lambda_{e p} T}{2} \sum_{n} \frac{\Phi_{n} D\left(i \omega_{m-n}\right)}{\sqrt{\Phi_{n}^{2}+\left(\omega_{n}+\Sigma_{n}\right)^{2}}} .
\end{aligned}
$$

This set of equations is solved for a given $D\left(i \omega_{m}\right)$ which by itself is only very weakly affected by fermions. In the normal state and at $\omega \ll \omega_{0}$, the self-energy Eq.32 reduces to the first term in Eq.31.

We have reviewed the derivation of these well known equations to underline the physical origin of the applicability of the Eliashberg approach. We see that the electron-phonon interaction gives rise to two physically distinct classes of interaction processes that contribute very differently to the fermionic self-energy and vertex corrections. In the first class, fast electrons are forced to vibrate at slow phonon frequencies (i.e., phonons are in resonance, but electrons are far from resonance). This gives rise to both vertex and self-energy corrections, but these are small $\left(\propto v_{s} / v_{F}\right)$ and are neglected in the Eliashberg theory. In the second class, phonons mediate an effective coupling between fermions and their zerosound collective modes. This process involves fermions with energies near resonance and phonons far away from resonance, and yields a strong renormalization of the fermionic propagator. The applicability of the Eliashberg approach is then based on the fact that one has to include the influence of phonon-mediated scattering on zero-sound vibrations, but can neglect the direct scattering of electrons by phonons.

These insights into the origin of the applicability of the Eliashberg theory will now be used to justify a generalized Eliashberg approach for spin mediated pairing.

\section{STRONG COUPLING APPROACH TO SPIN-FERMION INTERACTION}

As shown in the previous section, for electron phonon interactions the smallness of vertex and velocity renormalizations is caused by the small ratio of the Bose velocity and the Fermi velocity. The spin problem is qualitatively different. The bare spin-fluctuation propagator, Eq.8, describes propagating magnons whose velocity $c$ is expected to be of the same order as the Fermi velocity. There is then no a priori reason to neglect vertex and velocity renormalizations. Fortunately, this argument is not correct for the following reasons: First, as we just found in case of electron-phonon interaction, the fermionic self-energy in the Eliashberg theory is insensitive to the ratio of sound and Fermi velocities. The small ratio of $v_{s} / v_{F}$ is only necessary to eliminate regular terms in the self-energy, which are due to real scattering by phonons. For these terms to be small it is sufficient that bosons are slow modes compared to fermions. Second, the dynamics of the spin fluctuations is drastically modified, compared to the ballistic behavior of Eq. 8. A strong spin-fermion interaction at low-energies changes the bosonic dynamics from propagating to diffusive. Diffusive modes have a different relationship between typical momenta and energies compared to ballistic ones, making them slow modes compared to electrons. Consequently, regular terms in the fermionic self-energy again become smaller than singular ones.

We will show that in dimensions between $d=2$ and $d=3, d \Sigma(\omega) / d \omega$ scales as $\lambda^{3-d}$ where, we recall, $\lambda \propto \xi$ is the dimensionless spin-fermion coupling, while $v_{F}^{-1} d \Sigma / d k$ remains non-singular at $\xi=\infty$ in $d>2$ and only logarithmically increases for $d=2$. This implies that an Eliashberg-type approach near a magnetic instability is fully justified for $d>2$ and is "almost" justified for $d=2$. In the latter case (which is the most interesting because of the cuprates), we will have to invoke an extra approximation (an extension to large $N$ ) to be able to perform calculations in a controllable way.

Our strategy is the following. We first establish that one can indeed develop a controlled approach to the spin fluctuation problem in the normal state, see also Ref. 99. Next we apply this theory to the pairing problem and show that there is indeed $d$-wave superconductivity caused by antiferromagnetic spin fluctuations. We discuss the value of $T_{c}$ near optimal doping and the properties of the superconducting state, particularly the new effects associated with the feedback from superconductivity on the bosonic propagator, since these distinguish between spin-mediated and phonon-mediated pairings.

\section{A. Normal state behavior}

For our normal state analysis we follow Ref. 01 and perform computations assuming that the Eliashberg theory is valid, analyze the strong coupling results, and then show that vertex corrections and $v_{F}^{-1} d \Sigma_{\mathbf{k}}(\omega=0) / d \mathbf{k}$ are relatively small at strong coupling.

We begin by obtaining the full form of the dynamical spin susceptibility as it should undergo qualitative changes due to interactions with fermions. The selfenergy for the spin susceptibility (that is the spin polarization operator) is given by the convolution of the two fermionic propagators with the momentum difference near $\mathbf{Q}$ and the Pauli matrices in the vertices. Collecting all combinatorial factors, we obtain:

$$
\begin{aligned}
\Pi_{\mathbf{q}}\left(i \omega_{m}\right)= & -8 \bar{g} \xi^{2} \int \frac{d^{2} k d \Omega_{m}}{(2 \pi)^{3}} G_{\mathbf{k}}\left(i \Omega_{m}\right) \\
& \times G_{\mathbf{k}+\mathbf{q}}\left(i \omega_{m}+i \Omega_{m}\right)
\end{aligned}
$$

Here $G_{\mathbf{k}}\left(i \omega_{m}\right)$ is a full fermionic propagator

$$
G_{\mathbf{k}}\left(i \omega_{m}\right)=\frac{1}{i \omega_{m}+\Sigma\left(i \omega_{m}\right)-\varepsilon_{\mathbf{k}}}
$$

and $\Sigma\left(i \omega_{m}\right)$ is the self energy which remains to be determined. In principle, even in Eliashberg theory, this self- 
energy depends on the momentum component along the Fermi surface. However, for computations of $\Pi_{\mathbf{Q}}\left(i \omega_{m}\right)$ this dependence can be safely neglected; both fermions in the fermion polarization bubble should be close to the Fermi surface, and hence the momentum integration is necessarily confined to a narrow region around hot spots.

The $2+1$ dimensional integral over momentum and frequency in Eq. 34 is ultraviolet divergent; this implies that its dominant piece comes from highest energies which we have chosen to be $O(\Lambda)$. This contribution, however, should not be counted as it is already incorporated into the bare susceptibility. In addition, $\Pi_{\mathbf{Q}}\left(i \omega_{m}\right)$ contains a universal piece which remains finite even if we extend the momentum and frequency integration to infinity. The most straightforward way to obtain this contribution is to first integrate over momentum and then over frequency, i.e. perform computations assuming an infinite momentum cutoff but keeping energies still finite. One can easily make sure that for this order of limits, the high-energy contribution is absent due to a cancellation of infinities, and the full $\Pi_{\mathbf{q}}\left(i \omega_{m}\right)$ coincides with the universal part. The integration is straightforward and on performing it we obtain

$$
\Pi_{\mathbf{Q}}\left(i \omega_{m}\right)=-\frac{\left|\omega_{m}\right|}{\omega_{\mathrm{sf}}}
$$

where the characteristic energy scale is the spin fluctuation frequency,

$$
\omega_{\mathrm{sf}}=\frac{\pi}{4} \frac{\left(v_{F} \xi^{-1}\right)^{2}}{\bar{g} \sin \phi_{0}} .
$$

We recall that $\phi_{0}$ is the angle between the Fermi velocities at the two hot spots separated by $\mathbf{q} \sim \mathbf{Q}$. In optimally doped cuprates, $\phi_{0} \approx \pi / 2$ and weakly depends on $\mathbf{q}$ as long as one is far from momenta connecting Fermi points along the zone diagonal. This implies that we can safely set $\phi_{0}=\pi / 2$ and obtain, on making use of Eq.10,

$$
\omega_{\mathrm{sf}}=\frac{\pi}{4} \frac{v_{F}^{2} \xi^{-2}}{\bar{g}}=\frac{3}{16} \frac{v_{F} \xi^{-1}}{\lambda}=\frac{9}{64 \pi} \bar{g} \lambda^{-2} .
$$

An analytical continuation of Eq. 36 to the real frequency axis yields $\Pi_{\mathbf{Q}}(\omega)=i|\omega| / \omega_{\mathrm{sf}}$. As we anticipated, the coupling to low-energy fermions gives rise to a finite decay rate for a spin fluctuation. As typical spin frequencies are of order $v_{F} \xi^{-1} \sim c \xi^{-1}$, at strong coupling, $\lambda \geq 1$, the induced damping term $\omega / \omega_{s f} \sim \lambda \omega /\left(v_{F} \xi^{-1}\right)$ is large compared to the frequency dependent $\omega^{2} /\left(c \xi^{-1}\right)^{2}$ term in the bare susceptibility, i.e., the interaction with low energy fermions changes the form of the spin dynamics from a propagating one to a relaxational one. Neglecting the bare frequency term, we obtain

$$
\chi_{\mathbf{q}}\left(\omega+i 0^{+}\right)=\frac{\alpha \xi^{2}}{1+\xi^{2}(\mathbf{q}-\mathbf{Q})^{2}-i \omega / \omega_{\mathrm{sf}}},
$$

The same purely relaxational dynamic spin susceptibility was introduced phenomenologically by Millis, Monien and Pines 34 to describe the spin dynamics observed in NMR experiments on the cuprates.

We emphasize that the polarization operator, Eq.36, is independent of the actual form of the fermionic self en$\operatorname{ergy} \Sigma_{\mathbf{k}_{\mathrm{hs}}}(\omega)$. The explanation of why a $\mathbf{k}$-independent fermionic self-energy does not affect the polarization bubble at a finite momentum transfer was given by Kadanoffl01 who analyzed a similar problem for phonons. He pointed out that the linearization of the fermionic energy around $k_{F}$ is equivalent to imposing an approximate Migdal sum rule on the spectral function $A(\mathbf{k}, \omega)=$ $(1 / \pi) \operatorname{ImG}(\mathbf{k}, \omega)$

$$
\int d \epsilon_{\mathbf{k}} A(\mathbf{k}, \omega)=1
$$

where $\epsilon_{\mathbf{k}}$, the bare fermionic dispersion relation, $=\mathbf{v}_{\mathbf{k}}$. $\left(\mathbf{k}-\mathbf{k}_{\mathrm{F}}\right)$ in our case. Expressing $G(\mathbf{k}, \omega)$ in terms of $A(\mathbf{k}, \omega)$ via the Kramers-Kronig relation and making use of Eq.40, one finds

$$
\Pi(\mathbf{q}, \omega)=-\frac{i \omega}{\omega_{\mathrm{sf}}} \int_{-\infty}^{\infty} d \omega^{\prime} \frac{d f\left(\omega^{\prime}\right)}{d \omega^{\prime}}+\mathcal{O}\left(\omega^{2}\right),
$$

where $f\left(\omega^{\prime}\right)$ is the Fermi function. Eq. 36 then follows from the fact that $f\left(\omega^{\prime}\right)$ is 1 at $\omega^{\prime}=-\infty$ and 0 at $\omega^{\prime}=+\infty$.

We next determine the fermionic self energy $\Sigma_{\mathbf{k}}(i \omega)$. In Eliashberg theory it is given by

$$
\Sigma_{\mathbf{k}}\left(i \omega_{m}\right)=-3 g^{2} \int \frac{d^{2} \mathbf{q} d \varepsilon}{(2 \pi)^{3}} \chi_{\mathbf{q}}(i \varepsilon) G_{\mathbf{k}+\mathbf{q}}\left(i \omega_{m}+i \varepsilon\right)
$$

Consider first the self-energy at a hot spot. Introducing $i \widetilde{\Sigma}_{n}=i \omega_{n}+\Sigma_{\mathbf{k}_{\mathrm{hs}}}\left(i \omega_{n}\right)$ and $\mathbf{q}-\mathbf{Q}=\tilde{\mathbf{q}}$, we obtain from Eq. 42

$$
\begin{aligned}
\Sigma_{\mathbf{k}_{\mathrm{hs}}}\left(i \omega_{m}\right)= & \frac{-3 \bar{g}}{(2 \pi)^{3}} \int \frac{d \omega_{m^{\prime}} d^{2} \tilde{q}}{\tilde{\mathbf{q}}^{2}+\xi^{-2}\left(1+\frac{\left|\omega_{m}\right|}{\omega_{\mathrm{sf}}}\right)} \\
& \times \frac{1}{i \widetilde{\Sigma}_{m+m^{\prime}}-v_{F} \tilde{q}}
\end{aligned}
$$

with $\tilde{\mathbf{q}}^{2}=\tilde{q}_{\|}^{2}+\tilde{q}_{\perp}^{2}$. The integration over the component $\tilde{q}_{\|}$, parallel to the velocity $\mathbf{v}_{\mathbf{k}_{\mathrm{hs}}}$ at $\mathbf{k}_{\mathrm{hs}}$, is elementary and yields

$$
\Sigma_{\mathbf{k}_{\mathrm{hs}}}\left(i \omega_{n}\right)=\frac{3 \bar{g}}{8 \pi^{2} v_{F}^{2}} \int d \omega_{m}^{\prime} d \tilde{q}_{\perp} \frac{1}{\sqrt{\tilde{q}_{\perp}^{2}+q_{0}^{2}}\left(\tilde{q}_{\perp}-i q_{E}\right)}
$$

where $q_{0}=\xi^{-1}\left(1+\frac{\left|\omega_{m}\right|}{\omega_{\mathrm{sf}}}\right)^{1 / 2}$ and $q_{E}=\widetilde{\Sigma}_{m+m^{\prime}} / v_{F}$.

We next assume, and then verify, that a typical $\tilde{q}_{\perp} \ll$ $q_{0} . \quad q_{\perp}^{2}$ in the first term can then be ignored, and the momentum integration yields 


$$
\begin{aligned}
\int d \tilde{q}_{\perp} \frac{1}{\sqrt{\tilde{q}_{\perp}^{2}+q_{0}^{2}}} \frac{1}{\tilde{q}_{\perp}-i q_{E}} & \approx \frac{1}{q_{0}} \int_{-\infty}^{\infty} d \tilde{q}_{\perp} \frac{1}{\left(\tilde{q}_{\perp}-i q_{E}\right)} \\
& =i \pi \operatorname{signq} \mathrm{E} \\
& =i \pi \operatorname{sign}\left(\omega_{\mathrm{m}}+\omega_{\mathrm{m}}^{\prime}\right)
\end{aligned}
$$

On substituting this result into Eq 44 and splitting the frequency integral into several pieces, depending on the sign of $\omega_{m}+\omega_{m}^{\prime}$, we obtain

$$
\Sigma_{\mathbf{k}_{\mathrm{hs}}}\left(i \omega_{m}\right)=i \lambda \int_{-\omega_{m}}^{0} d \omega_{m}^{\prime} \frac{1}{\sqrt{1+\left|\omega_{m}^{\prime}\right| / \omega_{\mathrm{sf}}}}
$$

where $\lambda$ is given by Eq 10 . The remaining frequency integration is elementary and yields

$$
\Sigma_{\mathbf{k}}\left(i \omega_{m}\right)=i \lambda \frac{2 \omega_{m}}{1+\sqrt{1+\frac{\left|\omega_{m}\right|}{\omega_{\mathrm{sf}}}}} .
$$

We now return to the approximation we made above, that a typical $\tilde{q}_{\perp} \ll q_{0}$. Since the typical values for $\tilde{q}_{\perp}$, i.e. those which dominate the integral in Eq.45, are of order $q_{E}$, the approximation is valid if $q_{0} \gg q_{E}$. Further, a typical internal frequency $\omega_{m}^{\prime}$ is of order $\omega_{m}$, hence $q_{E} \sim$ $\left|\omega_{m}+\Sigma\left(\omega_{m}\right)\right| / v_{F}$ and $q_{0} \sim\left(\omega_{s f} \xi^{2}\right)^{-1}\left(\left|\omega_{m}\right|+\omega_{\mathrm{sf}}\right)^{1 / 2} \sim$ $\bar{g}^{1 / 2}\left(\left|\omega_{m}\right|+\omega_{\mathrm{sf}}\right)^{1 / 2} / v_{F}$. At weak coupling, $\lambda \ll 1$, the condition $q_{0} \gg q_{E}$ implies that $\omega \ll \bar{g} / \lambda$, i.e., at energies smaller that $\bar{g}$, the approximation is well justified. At strong coupling and $\omega \ll \omega_{\mathrm{sf}}, q_{E} \approx \lambda \omega / v_{F}$, $q_{0} \sim \lambda \omega_{\text {sf }} / v_{F}$, and the criterion $q_{0} \gg q_{E}$ is again satisfied. Finally, at strong coupling and $\omega>\omega_{s f}, q_{E} \sim q_{0}(\sim$ $\left.\bar{g} \omega_{m}\right)^{1 / 2} / v_{F}$. In this limit, the approximation we made is qualitatively but not quantitatively correct. In order to develop a well controlled theoretical framework for this limit, Abanov et al.177.122 developed a controllable 1/N approach by extending the model to a large number of hot spots, $N$, in the Brillouin zone $(N=8$ in the physical case). This allows an expansion in terms of $1 / N$. Alternatively, one can extend the model to a large number of fermion flavors, $M$, and expand in $1 / M$. For large $N$, $q_{0} / q_{E} \sim N^{2}$, i.e. the approximation $q_{0} \gg q_{E}$ is justified. Another appealing feature of this $\frac{1}{N}$ expansion is that within it, vertex corrections and the dependence of the self-energy of the momentum component transverse to the Fermi surface are also small in $\frac{1}{N}$ and can be computed systematically together with the corrections to the frequency dependent part of the self energy 51 . To keep our discussion focused on the key results, we will not discuss further the details of the $1 / N$ approach. Rather, we just emphasize that (i) Eq.47 is quantitatively correct even if $q_{0} \sim q_{E}$ and (ii) numerically the difference between approximate and more involved "exagt" results for the fermionic $\Sigma_{\mathbf{k}_{\mathrm{hs}}}(\omega)$ is only few percent 51 .

We next analyze the functional form of Eq.47. We see that $\Sigma_{\mathbf{k}_{\mathrm{hs}}}(i \omega)$ scales with $\lambda$ and at strong coupling exceeds the bare $i \omega$ term in the inverse fermion propagator $G_{\mathbf{k}_{\mathrm{hs}}}^{-1}(i \omega)=i \omega-\Sigma_{\mathbf{k}_{\mathrm{hs}}}(i \omega)$. As was the case with phonons, it originates in the scattering on zero-sound vibrations of the electronic subsystem, while spin-fluctuations mediate the interaction between electrons and their zero-sound fluctuations. Further, $\Sigma_{\mathbf{k}_{\mathrm{hs}}}(i \omega)$ evolves at the same typical energy $\omega_{\mathrm{sf}}$ as the bosonic self-energy. This interconnection between bosonic and fermionic propagators is one of the key "fingerprints" of the spin-fermion model.

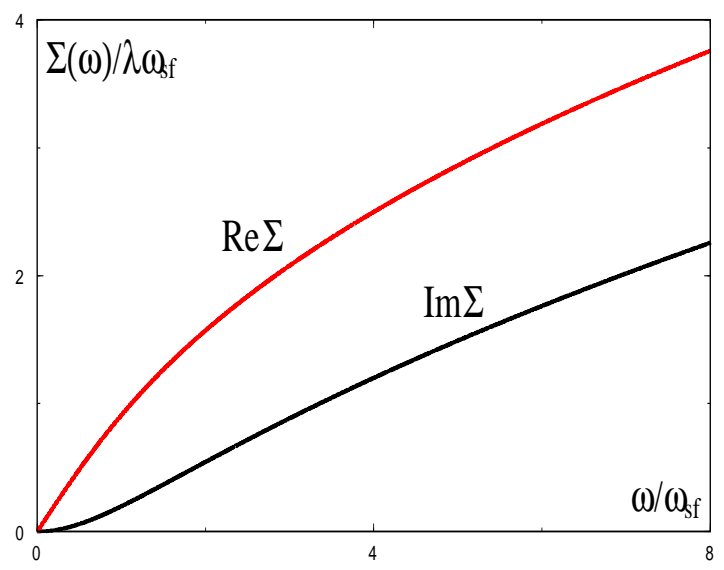

FIG. 8. The frequency dependence of the normal state electron self energy due to spin fluctuation-fermion interaction for a quasi particle at a hot spot (from Ref. 51).

In Fig 9 we show the behavior of the quasiparticle spectral function $A\left(\epsilon_{\mathbf{k}}, \omega\right)$ at various $\epsilon_{\mathbf{k}}$. We see that the spectral weight of the quasiparticle peak rapidly decreases as $\epsilon_{\mathbf{k}}$ becomes larger than $\omega_{\mathrm{sf}}$.

For small frequencies, $\omega \ll \omega_{\text {sf }}$, the spin susceptibility can be approximated by its static form. For the fermionic self-energy we find after analytical continuation

$$
\Sigma_{\mathbf{k}_{\mathrm{hs}}}(\omega)=\lambda\left(\omega+\frac{i \omega|\omega|}{4 \omega_{\mathrm{sf}}}\right) ; \quad\left(\omega \ll \omega_{\mathrm{sf}}\right) .
$$

We see that the quasiparticle damping term, although quadratic in $\omega$ as it should be in a Fermi liquid, scales inversely with $\omega_{\text {sf }}$, not with the Fermi energy as in conventional metals. As $\omega_{\mathrm{sf}}$ vanishes at the critical point, the width of the Fermi liquid region, where damping is small compared to $\omega$, progressively shrinks as $\xi$ increases. The quasiparticle renormalization factor $d \Sigma_{\mathbf{k}_{\mathrm{hs}}}(\omega) /\left.d \omega\right|_{\omega=0}=$ $\lambda \propto \xi$ increases as the system approaches the magnetic quantum critical point. The quasiparticle $z$-factor simultaneously decreases as

$$
z_{\mathbf{k}_{\mathrm{hs}}}=\frac{1}{1+\left.\frac{\partial \Sigma_{\mathbf{k}_{\mathrm{hs}}}(\omega)}{\partial \omega}\right|_{\omega=0}}=\frac{1}{1+\lambda}
$$

and vanishes at criticality.

At frequencies above $\omega_{\mathrm{sf}}$, the imaginary part of the fermionic self-energy resembles a linear function of $\omega$ over a substantial frequency range up to about $8 \omega_{\mathrm{sf}}$, and then eventually crosses over to

$$
\Sigma_{\mathbf{k}_{\mathrm{hs}}}(i \omega)=i \operatorname{sign} \omega(\bar{\omega}|\omega|)^{1 / 2}
$$


where

$$
\bar{\omega}=4 \lambda^{2} \omega_{\mathrm{sf}}=\frac{9}{16 \pi} \bar{g} .
$$

Thus $\bar{\omega}$ remains finite as $\xi \rightarrow \infty$. At the critical point $\omega_{\text {sf }} \rightarrow 0$, the self energy displays the non-Fermi-liquid behavior of Eq. 50 down to $\omega=0$. A plot of the fermionic self-energy is presented in Fig.8. The intermediate quasilinear regime is clearly visible. Note also that the deviations from Fermi liquid behavior starts already at small $\omega \sim \omega_{\text {sf }} / 2$.

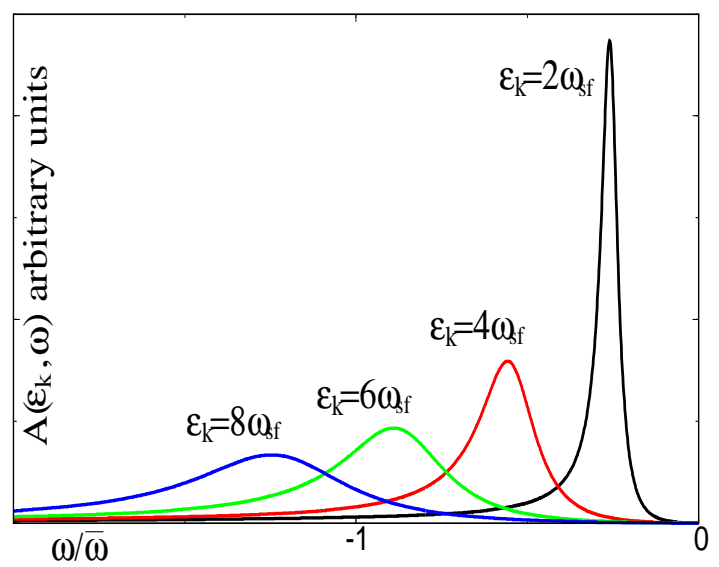

FIG. 9. The normal state spectral function. Note the absence of a quasiparticle peak. This is the consequence of the proximity to an antiferromagnetic quantum critical point. The figure is taken from Ref 51 .

We now consider how well the Eliashberg approximation is satisfied, i.e., whether vertex corrections and the momentum dependent piece in the fermionic self-energy are relatively small. To do this one needs to evaluate $\Sigma_{\mathbf{k}}(\omega=0)$ at $\mathbf{k} \neq \mathbf{k}_{\mathrm{hs}}$ and the vertex correction $\delta g / g$. The details of the derivation can be found in Ref. 51. We have

$$
\Sigma_{\mathbf{k}}(\omega=0)=\left[\frac{3}{4 \pi} \log \lambda\right] \varepsilon_{\mathbf{k}+\mathbf{Q}}
$$

and

$$
\frac{\delta g}{g}=\frac{Q\left(\phi_{0}\right)}{8} \log \lambda
$$

where $Q\left(\phi_{0}\right)=2 \phi_{0} / \pi$. For $\phi_{0} \approx \pi / 2, Q \approx 1$. We see that these two corrections depend only logarithmically on the coupling and at large $\lambda$ are parametrically small compared to the frequency dependence of the self-energy. Furthermore, at large $N$, both $\delta g / g$ and $\Sigma_{\mathbf{k}}$ contain an extra factor of $1 / N$, i.e., scale as $(1 / N) \log \lambda$. We see therefore that $(1 / N) \log \lambda$ is the analog of the second coupling constant $\tilde{\lambda}_{e p}$ for the phonon case. Just as for phonons, the applicability of the Eliashberg theory is related to the fact that this second coupling constant is much smaller than the primary coupling $\lambda$. In our case, this requires that $\lambda \gg(\log \lambda) / N$. We however emphasize that the smallness of the two "couplings" is not the result of the smallness of the velocity ratio but the consequence of the proximity to a critical point. Also, in distinction to phonons, the second coupling still diverges at the critical point and therefore corrections to Eliashberg theory cannot be neglected close to the antiferromagnetic transition. These corrections, however, have been analyzed within a renormalization group approach in Ref. 171,51 and found to be of minor relevance at intermediate coupling discussed here.

It is also instructive to explicitly compute the fermionic self-energy in the same way as we did in Section 3 for systems with electron-phonon interaction and verify that the reason for the dominance of the frequency dependence of the self energy is common to that for the phonon case. To see this, we introduce, by analogy with Eq. 27

$$
\Sigma\left(\mathbf{k}, i \omega_{m}\right)=\left(i \omega_{m}-\epsilon_{\mathbf{k}+\mathbf{Q}}\right) I\left(\mathbf{k}, i \omega_{m}\right)
$$

In the present case, $I\left(\mathbf{k}, i \omega_{m}\right)$ is singular as $\mathbf{k} \rightarrow \mathbf{k}_{\mathrm{hs}}$ and $\omega_{m} \rightarrow 0$, and $I\left(\mathbf{k}=\mathbf{k}_{\mathrm{hs}}, i \omega_{m} \rightarrow 0\right) \neq I\left(\mathbf{k} \rightarrow \mathbf{k}_{\mathrm{hs}}, 0\right) 51$. Evaluating $I\left(\mathbf{k}_{\mathrm{hs}}, i \omega_{m}\right)$ in the same way as in the phonon case, we find at $\omega \ll \omega_{s f}$

$$
I\left(\mathbf{k}_{\mathrm{hs}}, i \omega_{m}\right)=I_{r e g}+\lambda \frac{i \omega_{m}}{i \omega_{m}-\epsilon_{\mathbf{k}+\mathbf{Q}}},
$$

where $I_{\text {reg }}=O(\log \lambda)$. This form is the same as that found for phonons (see Eq. 30). The analogy implies that the dominant, $O(\lambda)$, contribution to the fermionic self-energy comes from magnetically mediated interactions between fermions and their zero sound excitations, whereas the actual spin-fermion scattering process in which fermions at forced to vibrate at typical spin frequencies yields a smaller $O(\log \lambda)$ contribution.

Finally, away from hot spots but still at the Fermi surface, the fermionic self-energy is given by the same expression, Eq. 47, as at a hot spot, but with a momentum dependent coupling constant $\lambda_{\mathbf{k}}$ and energy scale $\omega_{\text {sf }}(\mathbf{k})$ which obey

$$
\begin{aligned}
\lambda_{\mathbf{k}} & =\lambda /(1+\delta k \xi), \\
\omega_{\mathrm{sf}}(\mathbf{k}) & =\omega_{\mathrm{sf}}(1+\delta k \xi)^{2},
\end{aligned}
$$

where $\delta k=\left|\mathbf{k}_{\mathrm{F}}-\mathbf{k}_{\mathrm{hs}}\right|$ is the momentum deviation from a hot spot along the Fermi surface. We see that the effective coupling decreases upon deviation from a hot spot, while the upper energy scale for the Fermi liquid behavior increases. The increase of $\omega_{\text {sf }}$, however, is counterbalanced by the fact that $\omega_{\mathrm{sf}} \propto \sin \phi_{0}$, and $\phi_{0}$, which had set to be $\approx \pi / 2$, increases away from a hot spot 121 .

We see from Eq.56 that the width of the region where $\Sigma_{\mathbf{k}}(\omega)$ is independent of $\mathbf{k}$ (i.e., the "size" of a hot spot) depends on frequency. At the lowest frequencies, $\omega<\omega_{\text {sf }}(\mathbf{k}), \Sigma_{\mathbf{k}}(\omega)=\lambda_{\mathbf{k}} \omega$, and the hot spot physics is confined to a region of width $\xi^{-1}$ which progressively shrinks as $\xi$ increases. However, at frequencies above 
$\omega_{\mathrm{sf}}(\mathbf{k}), \Sigma_{\mathbf{k}}(\omega) \sim \lambda_{\mathbf{k}}\left(\omega \omega_{\mathrm{sf}}(\mathbf{k})^{1 / 2}=\lambda\left(\omega \omega_{\mathrm{sf}}\right)^{1 / 2}\right.$ is independent of k. Accordingly, physical processes that happen on these scales are isotropic (apart from the dependence on $\left.\phi_{0}\right)$. In this sense the whole Fermi surface acts as one big "hot spot".

One can easily perform the above analysis in dimensions larger than $d=2$. One finds that the quasiparticle spectral weight behaves for large $\lambda$ and the lowest energies $\omega<\omega_{\text {sf }}$ as

$$
z_{\mathbf{k}_{\mathrm{hs}}} \propto \lambda^{d-3}
$$

for $2<d<3$, and vanishes logarithmically for $d=3$. Correspondingly, in the quantum critical regime we find for the self energy along "hot lines"

$$
\Sigma_{\mathbf{k}_{\mathrm{hs}}}(i \omega) \propto i \omega|\omega|^{\frac{d-3}{2}},
$$

an expression that transforms into $\Sigma_{\mathbf{k}_{\mathrm{hs}}}(i \omega) \propto$ $i \omega \log (|\omega|)$ for $d=3$. Above $d=3$ no non-Fermi liquid effects result from the proximity to the quantum critical point. This demonstrates that many of the effects caused by the incoherent nature of the fermions are peculiar to $2 \mathrm{~d}$ and are considerably less pronounced in three dimensional systems.

\section{B. The $d_{x^{2}-y^{2}}$ pairing instability temperature}

We now consider the development of the pairing instability in the spin-fermion model. We follow Ref. 122. It is customary in an analysis of the pairing problem to introduce an infinitesimally small particle-particle vertex $\Phi_{\mathbf{k},-\mathbf{k}}^{(0)}(\omega,-\omega) \equiv \Phi_{\mathbf{k}}^{(0)}(\omega)$ and study its renormalization by the pairing interaction. The corresponding diagrams are presented in Fig. 10. The temperature at which the renormalized vertex diverges, i.e., when the equation for the full $\Phi_{\mathbf{k}}(\omega)$ has a nontrivial solution at vanishing $\Phi_{\mathbf{k}}^{(0)}(\omega)$, marks the onset of pairing.
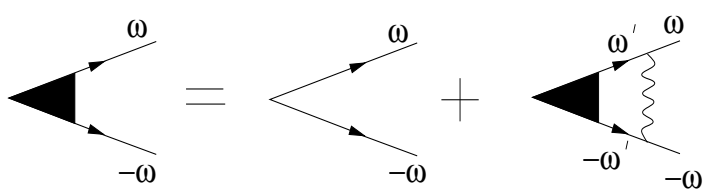

FIG 10. Diagrammatic representation for the pairing vertex 122 . The solid and wavy lines are fermionic and spin fluctuation propagators, respectively.

As noted above, the spin-mediated pairing interaction gives rise to $d_{x^{2}-y^{2}}$ superconductivity. We argued above that near the magnetic instability, the gap is maximum near the hot spots. One can check (see 122) that the pairing problem is confined to the hot regions in the sense that the momentum integration never extends to $\left|\mathbf{k}-\mathbf{k}_{\mathrm{hs}}\right|$ where the momentum dependence of the self-energy or infinitesimal pairing vertex becomes relevant. We can then assume that the pairing vertex is flat near the hot spots.
The underlying $d$-wave symmetry then implies that the gap changes sign between two hot regions separated by the antiferromagnetic $\mathbf{Q}$. A separation into hot and cold regions is indeed valid only if typical $\left|\mathbf{k}-\mathbf{k}_{\mathrm{hs}}\right| \leq k_{F}$. We will see below that the $d$-wave pairing problem is confined to frequencies of order $\bar{g}$. For these frequencies, the width of the hot region is finite and is constrained only by the requirement that $\omega_{\mathrm{sf}}(\mathbf{k})<\bar{g}$, a condition that implies (after a more accurate account of the overall factors) that the effective coupling is smaller than the fermionic bandwidth. As discussed in the Introduction, the latter is a necessary condition for the separation between high and low energies, and we assume it to hold. We comment below on what happens if typical $\left|\mathbf{k}-\mathbf{k}_{\mathrm{hs}}\right|>k_{F}$, i.e., hot and cold regions cannot be separated.

The value of the transition temperature depends sensitively on the behavior of fermions that are paired by the spin-mediated interaction. Our analysis of the normal state has shown that the character of the fermionic degrees of freedom changes at energies of order $\omega_{\text {sf }}$. For energies smaller than $\omega_{\text {sf }}$, fermions display Fermi liquid behavior, while at higher energies they display behavior that is different from that in a Fermi liquid. In the BCS theory of superconductivity only Fermi liquid degrees of freedom contribute to the pairing. Let us suppose that this also holds in the present case. Then the pairing problem would be qualitatively similar to that of BCS, since for frequencies smaller than $\omega_{\mathrm{sf}}$, the spin susceptibility that mediates pairing can be approximated by its static value. The linearized equation for the pairing vertex then has the form

$$
\Phi(\omega)=\frac{\lambda}{1+\lambda} \int_{T_{c}^{\mathrm{FL}}}^{\omega_{\mathrm{sf}}} d \omega^{\prime} \frac{\Phi\left(\omega^{\prime}\right)}{\omega^{\prime}},
$$

where the $1+\lambda$ factor in the denominator is the result of mass renormalization in the Fermi liquid regime $(\Sigma(\omega) \approx \lambda \omega)$. The solution of this equation 88 yields

$$
T_{\mathrm{c}}^{\mathrm{FL}} \sim \omega_{\mathrm{sf}} \exp \left(-\frac{1+\lambda}{\lambda}\right) .
$$

At weak coupling, this is just the BCS result. At strong coupling, the mass renormalization compensates the coupling constant, and $T_{\mathrm{c}}^{\mathrm{FL}}$ saturates at $T_{\mathrm{c}}^{\mathrm{FL}} \sim \omega_{\mathrm{sf}}$. This result, if correct, would imply that the pairing fluctuations become progressively less relevant as one approaches the quantum critical point $\xi^{-1} \rightarrow 0$ (see the left panel in Fig.5). At a first glance, this is what happens, because pairing of non Fermi-liquid degrees of freedom seems hard to accomplish. Indeed, at frequencies larger than $\omega_{\mathrm{sf}}$, the pairing interaction decreases as $\left(1+|\omega| / \omega_{\mathrm{sf}}\right)^{-1 / 2}$, and this apparently makes the frequency integral ultraviolet convergent, i.e., the "logarithmic" pairing problem does not appear to extend above $\omega_{\text {sf }}$. The flaw in this argument is that when the interaction decreases, the mass renormalization produced by the same interaction also decreases, and the large overall $\lambda$ is no longer compensated 
by $1+\Sigma(\omega) / \omega$. Indeed, for $\omega \gg \omega_{\text {sf }}, \Sigma(\omega)=(\bar{\omega} \omega)^{1 / 2}$, where, we recall, $\bar{\omega}=(9 / 16 \pi) \bar{g}$, and the mass renormalization is $1+(\bar{\omega} / \omega)^{1 / 2}=1+2 \lambda\left(\omega_{\text {sf }} / \omega\right)^{1 / 2} \ll \lambda$. Furthermore, we see that at frequencies between $\omega_{\text {sf }}$ and $\bar{\omega}$, the effective mass and effective interaction both scale as $(\omega)^{-1 / 2}$. The product of the two then scales as $1 / \omega$, i.e. the "logarithmic" pairing problem extends to frequencies of the order of $\bar{\omega}$ which, we recall, remains finite at $\xi=\infty$.

By itself, this effect does not guarantee that the pairing instability temperature is of order $\bar{\omega}$ as the pairing interaction depends on the transferred frequency $\omega-\omega^{\prime}$, and the linearized equation for the pairing vertex becomes an integral equation in frequency. In particular, for $\xi=\infty$, and hence $\omega_{\text {sf }}=0$, we need to solve

$$
\Phi(\omega)=\frac{1}{4} \int_{T_{\mathrm{cr}}}^{\bar{\omega}} d \omega^{\prime} \Phi\left(\omega^{\prime}\right) \frac{1}{\sqrt{\omega^{\prime}}} \frac{1}{\sqrt{\left|\omega-\omega^{\prime}\right|}}
$$

Observe that this equation does not have any adjustable parameter and is therefore fully universal when $T$ is expressed in units of $\bar{\omega}$. Eq. 61 has been anglyzed in detail by Finkel'stein, Abanov and one of us 122 . They found that it does have a nontrivial solution at

$$
T_{\text {cr }} \sim 0.2 \bar{\omega}
$$

They also analyzed the pairing problem at a finite $\lambda$ and found that incoherent fermions dominate pairing down to a surprisingly small $\lambda \sim 0.5$. The McMillan like formula, Eq.60, becomes valid only at smaller $\lambda$. A plot of $T_{\text {cr }}$ versus $\lambda$ is presented in Fig.11.

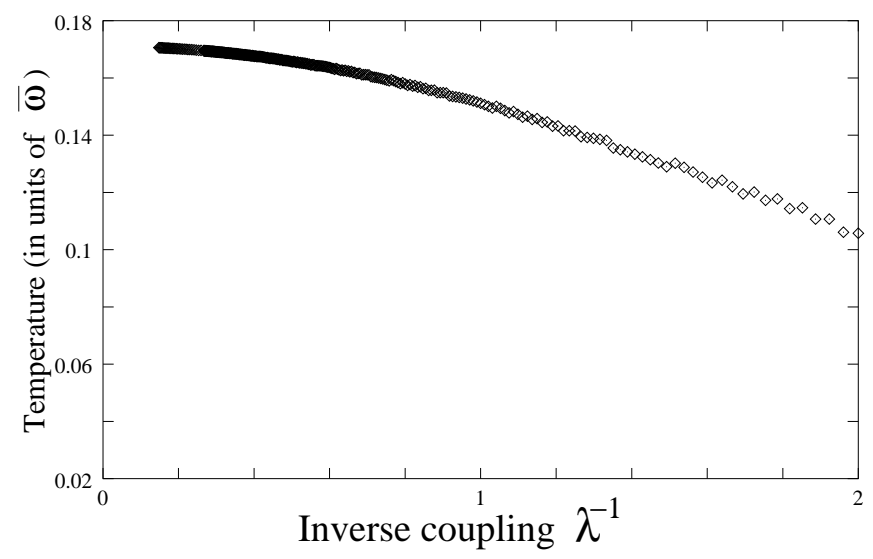

FIG. 11. The results for the instability temperature $T_{\text {cr }}$ obtained from the solution of the linearized Eliashberg equations for different values of the coupling constant $\lambda$. The figure is taken from Ref. 122 .

Several comments are in order here. First, at these values of the coupling constant (and doping) $T_{\text {cr }}$ does not coincide with the onset temperature for superconductivity, $T_{c}$, but rather represents the onset temperature for pseudogap behavior; the actual $T_{c}$ is lower, as discussed below. Second, we have neglected fluctuation effects due to the quasi-two dimensionality. The latter are expected to yield Kosterlitz-Thouless physics 100. Third, Eq.62, is only valid when $\bar{g}<W$ where we recall $W \sim v_{F}$ is the fermionic bandwidth. In the opposite limit $\bar{g}>W$, lattice effects are important and controlled analytical calculations are difficult to perform. One can, however, easily estimate that in this limit $T_{\text {cr }} \propto v_{F}^{2} / \bar{g} \propto \omega_{s f} \xi^{2}$. This estimate coincides with the result of Monthoux and Pines who extracted $T_{\text {cr }} \propto \omega_{s f} \xi^{2} \sim v_{F}^{2} / \bar{g}$ from their numerical analysis 38 . Since $v_{F}$ is proportional to the hopping matrix element, and $\bar{g}$ scales with the Hubbard $U$ at large $\xi$, it follows that $T_{\text {cr }} \propto J$, where $J$ is the magnetic exchange integral of the corresponding Heisenberg model which describes antiferromagnetism at half-filling.

Eq. 62 demonstrates that the $d$-wave pairing instability temperature of a two dimensional system at an antiferromagnetic quantum critical point is finite. It is interesting to note that the same holds for $p$-wave pairing at a ferromagnetjc-quantum critical point as shown by Roussev and Millis. 123 .

\section{Superconducting state}

We next extend the Eliashberg theory to the spin- fluctuation induced superconducting state. The discussion in this section follows Ref.102. We derive a generalized set of Eliashberg equations for the fermionic self-energy and the gap function that include an additional coupled equation for the spin polarization operator. The latter, as discussed in the Introduction, is produced by low-energy fermions and has to be determined self consistently.

As discussed above, the infinitesimal pairing vertex and the fermionic self-energy in the normal state depend weakly on momentum in the hot region $\left|\mathbf{k}-\mathbf{k}_{h s}\right| \leq \bar{g} / W$ where $W \propto v_{F}$ is the fermionic bandwidth. We will see that in the superconducting state, the momentum integration is also confined to hot regions. We can then safely neglect the weak momentum dependence of both $\Sigma(i \omega)$ and $\Phi(i \omega)$, as we did above in calculating $T_{\text {cr }}$. Subtle effects due to this weak momentum dependence will be considered in the next section. We will not attempt to discuss the behavior of the gap near the nodes. The latter is central for the interpretation of the experimental data at the lowest temperatures and frequencies, but not at energies comparable or larger than the maximum pairing gap. From our perspective it is not essential for the spin fluctuation induced pairing state.

\section{Generalized Eliashberg equations}

The derivation of the Eliashberg equations is straightforward. In the superconducting state, the normal and anomalous fermionic Green's functions $G_{\mathbf{k}}\left(i \omega_{n}\right)$ and $F_{\mathbf{k}}\left(i \omega_{n}\right)$ and the dynamical spin susceptibility are given 
by Eqs.11-13. It is convenient to rewrite $G_{\mathbf{k}}\left(i \omega_{n}\right)$ and $F_{\mathbf{k}}\left(i \omega_{n}\right)$ as

$$
\begin{aligned}
G_{\mathbf{k}}\left(i \omega_{n}\right) & =-\frac{\epsilon_{\mathbf{k}}+i \widetilde{\Sigma}_{n}}{\epsilon_{\mathbf{k}}^{2}+\widetilde{\Sigma}_{n}^{2}+\Phi^{2}\left(i \omega_{n}\right)}, \\
F_{\mathbf{k}}\left(i \omega_{n}\right) & =i \frac{\Phi\left(i \omega_{n}\right)}{\epsilon_{\mathbf{k}}^{2}+\widetilde{\Sigma}_{n}^{2}+\Phi^{2}\left(i \omega_{n}\right)} \\
F_{\mathbf{k}+\mathbf{Q}}\left(i \omega_{n}\right) & =-i \frac{\Phi\left(i \omega_{n}\right)}{\epsilon_{\mathbf{k}+\mathbf{Q}}^{2}+\widetilde{\Sigma}_{n}^{2}+\Phi^{2}\left(i \omega_{n}\right)}
\end{aligned}
$$

where $i \widetilde{\Sigma}_{n}=i \omega_{n}+\Sigma_{\mathbf{k}_{\mathrm{hs}}}\left(i \omega_{n}\right)$ (in real frequencies, $\widetilde{\Sigma}(\omega)=$ $\left.\omega+\Sigma_{\mathbf{k}_{\mathrm{hs}}}(\omega)\right)$. Without losing generality we can set $\epsilon_{\mathbf{k}}=v_{x} \tilde{k}_{x}+v_{y} \tilde{k}_{y}$ and $\epsilon_{\mathbf{k}+\mathbf{Q}}=-v_{x} \tilde{k}_{x}+v_{y} \tilde{k}_{y}$ where $\tilde{k}=k-k_{h s}$. The sign change between $F_{\mathbf{k}}$ and $F_{\mathbf{k}+\mathbf{Q}}$ is the result of $d_{x^{2}-y^{2}}$ symmetry. The spin susceptibility, we recall, is given by

$$
\chi_{\mathbf{q}}\left(i \omega_{m}\right)=\frac{\alpha \xi^{2}}{1+\xi^{2}(\mathbf{q}-\mathbf{Q})^{2}-\Pi_{\mathbf{Q}}\left(i \omega_{m}\right)} .
$$

We need to obtain the equations for the fermionic selfenergy $\Sigma_{\mathbf{k}_{\mathrm{hs}}}\left(i \omega_{m}\right)$, the anomalous vertex $\Phi_{\mathbf{k}_{\mathrm{hs}}}\left(i \omega_{m}\right)$, and the spin polarization operator $\Pi_{\mathbf{Q}}\left(i \omega_{m}\right)$. The spin polarization operator is obtained in the same way as in the normal state, but now there are two particle-hole bubbles: one is the convolution of $G_{\mathbf{k}} G_{\mathbf{k}+\mathbf{Q}}$ and the other is the convolution of $F_{\mathbf{k}} F_{\mathbf{k}+\mathbf{Q}}$. We have

$$
\begin{aligned}
\Pi_{\mathbf{Q}}\left(i \omega_{n}\right)= & -8 \bar{g} \xi^{2} T \sum_{m} \int \frac{d^{2} k}{(2 \pi)^{2}}\left[G_{\mathbf{k}+\mathbf{Q}}\left(i \omega_{n+m}\right)\right. \\
& \left.\times G_{\mathbf{k}}\left(i \omega_{m}\right)-F_{\mathbf{k}+\mathbf{Q}}\left(i \omega_{n+m}\right) F_{\mathbf{k}}\left(i \omega_{m}\right)\right]
\end{aligned}
$$

(the negative sign between the two terms originates in the summation over the spin components). The momentum integration can be performed explicitly and yields

$$
\begin{aligned}
\Pi_{\mathbf{Q}}\left(i \omega_{n}\right)= & -\frac{4 \bar{g} \xi^{2}}{v_{F}^{2}} T \sum_{m}\left[1-g\left(i \omega_{m}\right) g\left(i \omega_{n+m}\right)\right. \\
& \left.-f\left(i \omega_{m}\right) f\left(i \omega_{n+m}\right)\right]
\end{aligned}
$$

where

$$
g\left(i \omega_{m}\right)=\frac{\widetilde{\Sigma}_{m}}{\sqrt{\widetilde{\Sigma}_{m}^{2}+\Phi^{2}\left(i \omega_{m}\right)}}
$$

and

$$
f\left(i \omega_{m}\right)=\frac{\Phi\left(i \omega_{m}\right)}{\sqrt{\tilde{\Sigma}_{m}^{2}+\Phi^{2}\left(i \omega_{m}\right)}}
$$

The first term in Eq.66 is the result of the regularization of the ultraviolet singularity. The additional sign change between $g g$ and $f f$ terms in Eq.66 is due to the $d$-wave form of $F_{\mathbf{k}}$.
Eq.66 takes into account the change of the low energy spin dynamics in the pairing state. In the case of electron-phonon interaction a corresponding change of the phonon dynamics exists as well, causing a shift of the phonon frequency and line width below $\mathrm{T}_{c}$. While this effect is only a minor correction to the phononic dynamics and is often neglected124, it leads to a dramatic change of the spin dynamics in our case.

The other two equations are formally the same as for phonon-mediated superconductors. The fermionic selfenergy $\Sigma\left(\omega_{n}\right)$ is given by

$$
\Sigma\left(i \omega_{n}\right)=3 g^{2} T \sum_{m} \int \frac{d^{2} q}{(2 \pi)^{2}} \chi\left(\mathbf{q}, i \omega_{m}\right) G_{\mathbf{k}_{\mathrm{hs}}+\mathbf{q}}\left(\omega_{n+m}\right)
$$

Performing the momentum integration along the same lines as in the normal state calculations we find

$$
\Sigma\left(i \omega_{n}\right)=\frac{3 g^{2}}{2 v_{F}} T \sum_{m} D\left(i \omega_{m}\right) g\left(i \omega_{n}+i \omega_{m}\right)
$$

where $D$ is the effective bosonic propagator that is obtained by integrating the dynamical spin susceptibility over the momentum component along the Fermi surface and setting other momentum components to $\mathbf{Q}$ (the last step is equivalent to the approximation we discussed below Eq.45). We have

$$
\begin{aligned}
D\left(i \omega_{m}\right) & =\left.\int \frac{d q_{\|}}{2 \pi} \chi\left(\mathbf{q}, i \omega_{m}\right)\right|_{q_{\perp}=Q} \\
& =\frac{\alpha \xi}{2 \sqrt{1-\Pi_{\mathbf{Q}}\left(i \omega_{m}\right)}}
\end{aligned}
$$

An analogous equation is obtained for the anomalous vertex

$$
\Phi\left(i \omega_{n}\right)=\frac{3 g^{2}}{2 v_{F}} T \sum_{m} D\left(i \omega_{m}\right) f\left(i \omega_{n}+i \omega_{m}\right) .
$$

Eqs. 66, 70, and 72 constitute the full set of Eliashberg equations for the spin-mediated superconductivity. Alternatively to $\Phi(\omega)$ and $\Sigma(\omega)$ we can also introduce

$$
Z(\omega)=1+\frac{\Sigma(\omega)}{\omega} \quad \Delta(\omega)=\frac{\Phi(\omega)}{Z(\omega)}
$$

The complex function $\Delta(\omega)$ reduces to the superconducting gap $\Delta$ in BCS theory where we also have $Z(\omega)=1$. In Eliashberg theory, the superconducting gap, defined as a frequency where the density of states has a peak, is the solution of $\Delta(\omega=\Delta)=\omega$.

We again emphasize that the Eliashberg equations are valid for fermionic momenta which deviate from hot spots by less than $\bar{g} / v_{F}$. For these momenta, the pairing vertex can be approximated by a k-independent function which however changes sign between two hot regions separated by $\mathbf{Q}$. For larger deviations, the anomalous vertex rapidly goes down and eventually vanishes along zone diagonals. 


\section{Solution of the Eliashberg equations:}

We discuss the general structure of the solutions of the set of Eliashberg equations, and then present the results of their numerical solution. First, we see from Eq. 66 that, as in the normal state, $\Pi(\omega=0)=0$ for any $\Sigma(\omega)$ and $\Phi(\omega)$. This physically implies that the development of the gap does not change the magnetic correlation length. This result becomes evident if one notices that $d$-wave pairing involves fermions from opposite sub-lattices. Second, the opening of the superconducting gap changes the low frequency spin dynamics. Now quasiparticles near hot spots are gapped, and a spin fluctuation can decay into a particle-hole pair only when it can pull two particles out of the condensate of Cooper pairs. This implies that the decay into particlehole excitations is only possible if the external frequency is larger than $2 \Delta$. At smaller frequencies, we should have $\Pi^{\prime \prime}(\omega)=0$ at $T=0127,170$. This result indeed readily follows from Eq. 66. The Kramers-Kronig relation $\Pi^{\prime}(\omega)=(2 / \pi) \int_{0}^{\infty} \Pi^{\prime \prime}(x) /\left(x^{2}-\omega^{2}\right)$ then implies that because of a drop in $\Pi^{\prime \prime}(\omega)$, the spin polarization operator in a superconductor acquires a real part, which at low $\omega$ is quadratic in frequency and is of order $\omega^{2} /\left(\Delta \omega_{\mathrm{sf}}\right)$. Substituting this result into Eq.13, we find that at low energies, spin excitations in a $d$-wave superconductor are propagating, gapped magnon-like quasiparticles

$$
\chi(\mathbf{q}, \omega) \propto \frac{\Delta_{s}^{2}}{\Delta_{s}^{2}+c_{s}^{2}(\mathbf{q}-\mathbf{Q})^{2}-\omega^{2}} .
$$

where

$$
\Delta_{s} \sim\left(\Delta \omega_{\mathrm{sf}}\right)^{1 / 2}
$$

and $c_{s}^{2} \sim v_{\mathrm{F}}^{2} \Delta / \bar{g}$. The re-emergence of propagating spin dynamics implies that the dynamical spin susceptibility acquires a resonance peak which at $\mathbf{q}=\mathbf{Q}$ is located at $\omega=\Delta_{s}$.

Eq.74 is indeed meaningful only if $\Delta_{s} \leq \Delta$, i.e., $\omega_{\text {sf }} \leq \Delta$. Otherwise the use of the quadratic form for $\Pi(\omega)$ is not justified. To find out how $\Delta$ depends on the coupling constant, one needs to carefully analyze the full set of Eqs.66.72. This analysis is rather involved 122.102 , and is not directly related to the goal of this Chapter. We skip the details and quote the result. It turns out that at strong coupling, $\lambda \geq 1$, i.e. for optimally and underdoped cuprates, the condition $\Delta>\omega_{\text {sf }}$ is satisfied: the gap scales with $\bar{\omega}$ and saturates at $\Delta \approx 0.35 \bar{\omega}=0.06 \bar{g}$ at $\lambda \rightarrow \infty$, while $\omega_{\text {sf }} \propto \lambda^{-2} \rightarrow 0$. In this situation, the spin excitations in a superconductor are propagating, particlelike modes with a gap $\Delta_{s}$. However, in distinction to phonons, these propagating magnons get their identity from a strong coupling feedback effect in the superconducting state.

At weak coupling, the superconducting problem is of the BCS type, and $\Delta \ll \omega_{\text {sf }}$. This result is intuitively obvious as $\omega_{\text {sf }}$ plays the role of the Debye frequency in the sense that the bosonic mode that mediates pairing decreases at frequencies above $\omega_{\text {sf }}$. For $\Delta \ll \omega_{s f}, \chi(\mathbf{Q}, \omega)$ does not have a pole at frequencies where $\Pi(\omega) \propto \omega^{2}$. Still, a pexpen at weak coupling $127130,170,156$. To see this, note that at $\omega \approx 2 \Delta$ one can simultaneously set both fermionic frequencies in the bubble to be close to $\Delta$, and make both propagators singular due to the vanishing of $\sqrt{\tilde{\Sigma}^{2}-\Phi^{2}}$ where, we recall, $\tilde{\Sigma}=\omega+\Sigma(\omega)$. Substituting $\tilde{\Sigma}^{2}(\omega)-\Phi^{2}(\omega) \propto \omega-\Delta$ into Eq. 66 and using the spectral representation, we obtain for $\omega=2 \Delta+\epsilon$

$$
\Pi^{\prime \prime}(\omega) \propto \int_{0}^{\epsilon} \frac{d x}{(x(\epsilon-x))^{1 / 2}} .
$$

Evaluating the integral, we find that $\Pi^{\prime \prime}$ undergoes a finite jump at $\omega=2 \Delta$. By the Kramers-Kronig relation, this jump gives rise to a logarithmic singularity in $\Pi^{\prime}(\omega)$ at $\omega=2 \Delta$ :

$$
\Pi^{\prime}(\omega)=\frac{2}{\pi} \int_{2 \Delta}^{\infty} d x \frac{\Pi^{\prime \prime}(x)}{x^{2}-\omega^{2}} \propto \Delta \log \frac{2 \Delta}{|\omega-2 \Delta|} .
$$

The behavior of $\Pi^{\prime}(\omega)$ and $\Pi^{\prime \prime}(\omega)$ is schematically shown in Fig.12. The fact that $\Pi^{\prime}(\omega)$ diverges logarithmically at $2 \Delta$ implies that no matter how small $\Delta / \omega_{\text {sf }}$ is, $\chi(\mathbf{Q}, \omega)$ has a pole at $\Delta_{s}<2 \Delta$, when $\Pi^{\prime \prime}(\omega)$ is still zero. Simple estimates show that for weak coupling, where $\omega_{\text {sf }} \gg \Delta$, the singularity occurs at $\Delta_{s}=2 \Delta\left(1-Z_{s}\right)$ where $Z_{s} \propto e^{-\omega_{\text {sf }} /(2 \Delta)}$ is also the spectral weight of the resonance peak in this limit.

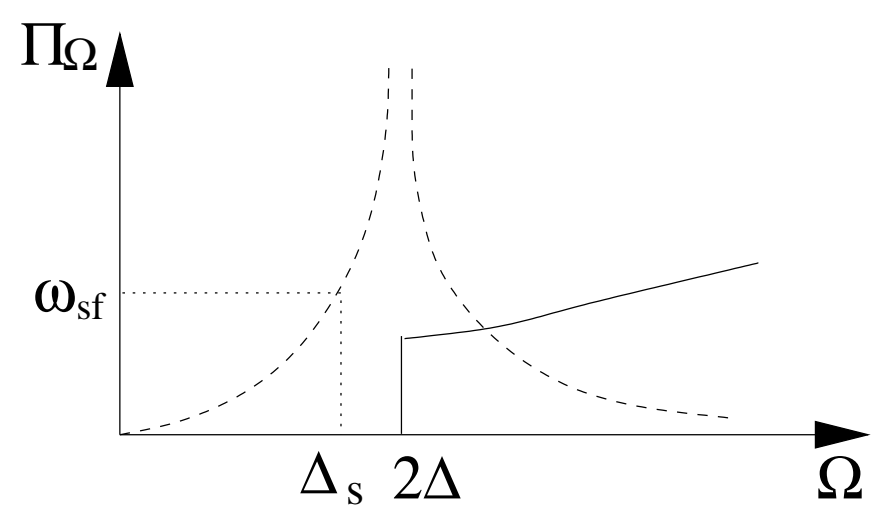

FIG. 12. Schematic behavior of the real (dashed line) and imaginary (solid line) parts of the particle hole bubble in the superconducting state. Due to the discontinuous behavior of $\Pi^{\prime \prime}(\omega)$ at $\omega=2 \Delta$, the real part $\Pi^{\prime}(\omega)$ is logarithmically divergent at $2 \Delta$. For small $\omega$, the real part behaves like $\omega^{2} / \Delta$. The figure is taken from 149 .

We see therefore that the resonance in the spin susceptibility exists both at weak and at strong coupling. At strong coupling, the resonance frequency is $\Delta_{s} \sim \Delta / \lambda \ll$ $\Delta$, i.e., the resonance occurs in the frequency range where spin excitations behave as propagating magnon-like excitations. At weak coupling, the resonance occurs very near $2 \Delta$ due to the logarithmic singularity in $\Pi^{\prime}(\omega)$. In 
practice, however, the resonance at weak coupling can hardly be observed because the residue of the peak in the spin susceptibility $Z_{s}$ is exponentially small.

Fig. 13 shows the results for $\chi(\mathbf{Q}, \omega)$ obtained from the full solution of the set of three coupled equations at $T \approx 0$ and three different coupling constant 102 . For $\lambda \geq 1$, the spin susceptibility has a sharp peak at $\omega=\Delta_{s}$. The peak gets sharper when it moves away from $2 \Delta$. At the same time, for $\lambda=0.5$, corresponding to weak coupling, the peak is very weak and is washed out by a small thermal damping. In this case, $\chi^{\prime \prime}$ only displays a discontinuity at $2 \Delta$.

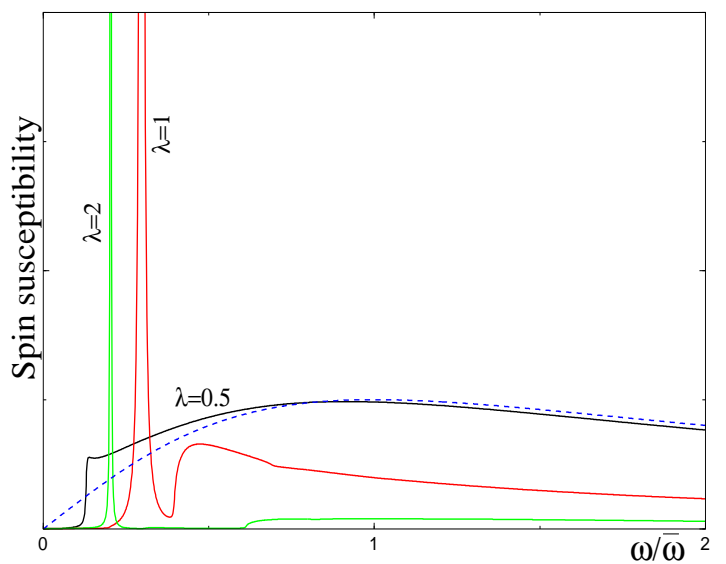

FIG. 13. Imaginary part of the dynamical spin susceptibility in the superconducting state at $T \ll T_{c}$ obtained from the solution of the set of three Eliashberg equations for coupling constants $\lambda=0.5, \lambda=1$, and $\lambda=2$. The figure is taken from 149 .

We next show that the resonance peak does not exist for $s$-wave superconductors 131 . In the latter case, the spin polarization operator is given by almost the same expression as in Eq. 66, but with a different sign of the $f f$-term; recall that the original sign in Eq.(66) originated from the fact that the two fermions in the spin polarization bubble differ in momentum by $\mathbf{Q}$, and the $d$-wave gap changes sign under $\mathbf{k} \rightarrow \mathbf{k}+\mathbf{Q}$. One can immediately check that for a different sign of the anomalous term, $\Pi^{\prime \prime}$ is continuous at $2 \Delta$. Accordingly, $\Pi^{\prime}(\omega)$ does not diverge at $2 \Delta$, and hence there is no resonance at weak coupling. Still, however, one could expect the resonance at strong coupling as at small frequencies $\Pi^{\prime}(\omega)$ is quadratic in $\omega$ by virtue of the existence of the threshold for $\Pi^{\prime \prime}$. It turns out, however, that for $s$-wave pairing the resonance is precluded by the fact that $\Pi(\omega=0)$ changes between the normal and the superconducting states.

One can make sure that in an $s$-wave superconductor, $\Pi(\omega=0)<0$, and that this negative term overshadows the positive $\omega^{2}$ term in $\Pi(\omega)$ in such a way that for all frequencies below $2 \Delta, \Pi(\omega)<0$ and hence the resonance simply does not exist. That $\Pi(\omega=0)<0$ in $s$-wave superconductors can be easily explained: a negative $\Pi(0)$ implies that the spin correlation length decreases as the system becomes superconducting. This is exactly what one should expect as $s$-wave pairing involves fermions both from different sub-lattices as well as from the same sub-lattice. The pairing of fermions from the same sublattice into a spin-singlet state obviously reduces the antiferromagnetic correlation length.

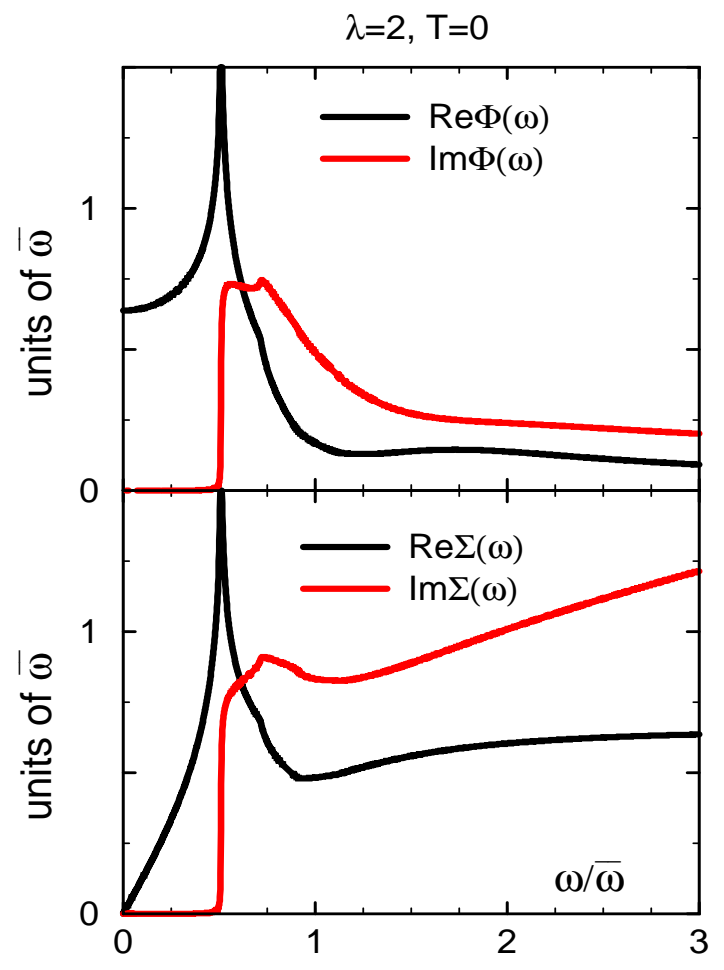

FIG. 14. The real and imaginary parts of the fermionic self-energy $\Sigma(\omega)$ and the pairing vertex $\Phi(\omega)$ for $\lambda=2$ and the lowest $T$. The results are from Ref. 132 .

We also comment on the dispersion of the resonance peak. In Eq. 74 we assumed that $\Delta_{s}$ is a constant. In fact, $\Delta_{s}$ depends on $\mathbf{q}$ since for any given $\mathbf{q}, \Delta_{s}^{2} \propto \Delta(\mathbf{q})$ where $\Delta(\mathbf{q})$ is a $d$ - wave gap at the points at the Fermi surface which are connected by q. In particular, $\Delta_{s}$ should vanish at $\mathbf{q}=\mathbf{Q}_{\text {min }}$ which connects the nodal points. This effect accounts for the "negative" dispersion of the resonance peak 128 131. The latter certainly overshadows the positive dispersion due to $(\mathbf{q}-\mathbf{Q})^{2}$ term for $\mathbf{q}$ close to $\mathbf{Q}_{\text {min }}$ and may do so even for $\mathbf{q}$ near $\mathbf{Q}$ if the correlation length is not large enough. This effect is, however, not a part of the quantum-critical description (it should become progressively less relevant for $\mathbf{q} \neq \mathbf{Q}_{\text {min }}$ when $\xi$ increases), and we ignore it in the subsequent analysis. Note, however, that the negative dispersion of the peak implies that the peak exists only for a small range of momenta between $\mathbf{Q}$ and $\mathbf{P}_{\text {mim }}$ In optimally doped cuprates, $\mathbf{Q}_{\min } \approx(0.8 \pi, 0.8 \pi) 151.153$, and the momentum range for the peak does not exceed $6 \%$ of the Brillouin zone. The actual $\mathbf{q}$ region where the peak is observable is even smaller as the intensity of the peak also decreases when $\mathbf{q}$ approaches $\mathbf{Q}_{\min }$. The smallness of the $\mathbf{q}$-range for the peak accounts for small overall spectral 
intensity $I_{0}=\int S(\mathbf{q}, \omega) d^{2} q d \omega /(2 \pi)^{3}$ that turns out to be substantially smaller than $S(S+1) / 3=1 / 4$. Still, at $\mathbf{Q}$, the intensity of the peak is not small (experimentally, $\int S(\mathbf{Q}, \omega) d \omega \sim 1.5$ in optimally doped YBCO 161.162 ), and we have verified that for the frequencies that we consider below, the typical $\mathbf{q}-\mathbf{Q}$ that account for the feedback on the fermions are well within the $\mathbf{q}$ range between $\mathbf{Q}$ and $\mathbf{Q}_{\min }$. In other words, the small overall intensity of the resonance peak does not preclude strong feedback effects from the resonance peak on fermionic variables.

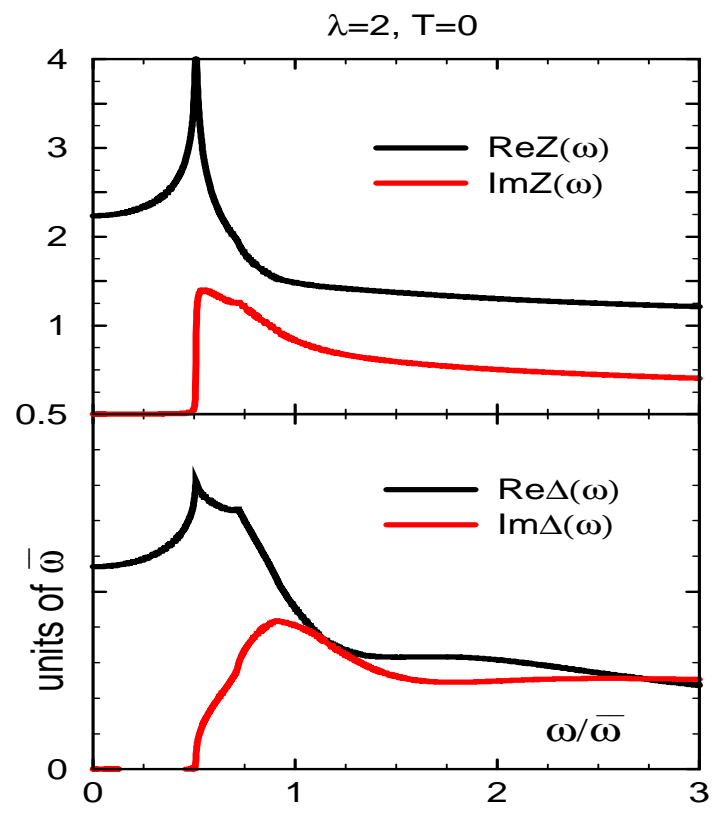

FIG. 15. The real and imaginary parts of the effective gap $\Delta(\omega)$ and the quasiparticle renormalization factor $Z(\omega)$ for $\lambda=2$ and the lowest $T$. The results are from Ref. 132 .

For completeness, in Figs.14 and 15 we present results for the fermionic self-energy and the pairing vertex for the smallest $T$. We see that the real parts of $\Phi(\omega)$ and $\Delta(\omega)$ are finite at $\omega=0$ as should be the case in the superconducting state. The imaginary parts of $\Phi(\omega)$ and $\Sigma(\omega)$ (and of $\Delta(\omega)$ and $Z(\omega)$ ) vanish at small frequencies and appear only above the threshold frequency that is precisely $\Delta+\Delta_{s}$. Furthermore, all variables have a complex internal structure at large frequencies. In the next section we discuss the physical origin of the threshold at $\Delta+\Delta_{s}$ and also show that one can extract $3 \Delta$ from the derivative of $\Sigma^{\prime \prime}(\omega)$.

Few words about the numbers. For $\lambda=2, \Delta \approx 0.3 \bar{\omega}$ and $\Delta_{s} \approx 0.2 \bar{\omega}$, i.e., $\Delta$ and $\Delta_{s}$ are comparable to each other. For $\lambda \gg 1$ a numerical solution of the Eliashberg equations leads to $\Delta \sim 2 T_{c r} \sim 0.35 \bar{\omega}$, and $\Delta_{s} \sim 0.25 \bar{\omega} / \lambda \ll \Delta$.

\section{FINGERPRINTS OF SPIN FERMION PAIRING}

In this Section, we discuss the extent to which the "fingerprints" of spin-mediated pairing can be extracted from experiments on materials that are candidates for a magnetically-mediated superconductivity. Due to strong spin-fermion coupling, there is unusually strong feedback from spin excitations on fermions, specific to $d$-wave superconductors with a magnetic pairing interaction. The origin of this feedback is the emergence of a propagating collective spin bosonic mode below $T_{c r}$. This mode is present for any coupling strength, and its gap $\Delta_{s}$ is smaller than the minimum energy $\sim 2 \Delta$ that is necessary to break a Cooper pair. In the vicinity of the antiferromagnetic phase, $\Delta_{s} \propto \xi^{-1}$ where $\xi$ is the magnetic correlation length. We show that this propagating spin mode changes the onset frequency for single particle scattering, gives rise to the "peak-dip-hump" features in the quasiparticle spectral function, the "dippeak" features in tunneling SIS and SIN conductances, and to singularities and fine structures in the optical conductivity. In section 6 , we apply these results to cuprate superconductors and aroue that (i) these features have beon - pbserved 147, 148, 151, 153, 154, $157,158,160$ (ii) ARPES 147 148, 151, 153 , tunneling 154, 157, and conductivity data 158,160 are consistent with each other, and (iii) the value of $\Delta_{s}$ extracted from these various experiments coincides with the resonance frequency measured directly in neutron scattering experiments161 163.

\section{A. The physical origin of the effect}

The physical effect that accounts for dips and humps in the density of states and spectral function of cuprates is not new and is known for conyentional $s$-wave superconductors as the Holstein effect $5.164,165$.
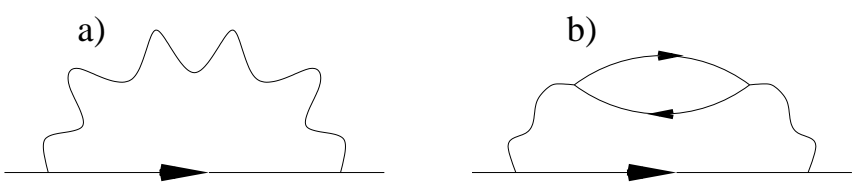

FIG. 16. a) The exchange diagram for a boson mediated interaction. The solid line stands for a propagating fermion. The wiggly line is a phonon propagator in the case of electron- phonon interaction, and a magnon line in the case of a spin- fluctuation mediated interaction. b) The lowest order diagram for the fermionic self energy due to a direct four fermion interaction, also represented by a wiggly line. The figure is taken from 149 .

Consider a clean $s$-wave superconductor, and suppose that the residual interaction between fermions occurs via the exchange of an Einstein phonon. Assume for simplicity that the fully renormalized electron phonon coupling is some constant $g_{\mathrm{ep}}$, and that the phonon propagator 
$D(q, \omega)$ is independent of momentum $q$ and has a sinole at a phonon frequency $\omega_{0}$ (the Holstein model) 164 166. Phonon exchange gives rise to a fermionic selfenergy (see Fig 16a)

$$
\Sigma\left(i \omega_{m}\right)=g_{\mathrm{ep}}^{2} T \sum_{n} \int \frac{d^{d} k}{(2 \pi)^{d}} G_{\mathbf{k}}\left(i \omega_{n}\right) D\left(i \omega_{m}-i \omega_{n}\right)
$$

which is a convolution of $D(\omega)=1 /\left(\omega_{0}^{2}-\left(\omega+i 0^{+}\right)^{2}\right)$ with the full fermionic propagator $G_{\mathbf{k}}(\omega)$, which in a superconductor is given by Eq.11:

$$
G_{\mathbf{k}}(\omega)=\frac{\tilde{\Sigma}(\omega)+\varepsilon_{\mathbf{k}}}{\tilde{\Sigma}(\omega)^{2}-\Phi^{2}(\omega)-\varepsilon_{\mathbf{k}}^{2}}
$$

As before, $\Phi(\omega)$ is the pairing vertex, and $\varepsilon_{\mathbf{k}}$ is the band dispersion of the fermions. At $T=0$ both $\Sigma^{\prime \prime}(\omega)$ and $\Phi^{\prime \prime}(\omega)$ obviously vanish for $\omega \leq \Delta$. This implies that the fermionic spectral function $A_{\mathbf{k}}(\omega)=\left|G_{\mathbf{k}}^{\prime \prime}(\omega)\right| / \pi$ for particles at the Fermi surface $\left(\mathbf{k}=\mathbf{k}_{\mathrm{F}}\right)$ has a $\delta$-function peak at $\omega=\Delta$, i.e. $\Delta$ is a sharp gap at zero temperature. The fermionic density of states in a superconductor

$$
N(\omega)=N_{0} \operatorname{Im}\left[\frac{\tilde{\Sigma}(\omega)}{\left(\Phi^{2}(\omega)-\tilde{\Sigma}^{2}(\omega)\right)^{1 / 2}}\right]
$$

vanishes for $\omega<\Delta$ and has a square-root singularity $N(\omega) \propto(\omega-\Delta)^{-1 / 2}$ for frequencies above the gap $\left(N_{0}\right.$ is the normal state density of states).

The onset of the imaginary part of the self-energy, Eq.78, can be easily obtained by using the spectral representation for fermionic and bosonic propagators in Eq. 78 and re-expressing the momentum integration in terms of an integration over $\varepsilon_{\mathbf{k}}$. At $T=0$ we obtain

$$
\Sigma^{\prime \prime}(\omega>0) \propto \int_{0}^{\omega} d \omega^{\prime} N\left(\omega^{\prime}\right) D^{\prime \prime}\left(\omega-\omega^{\prime}\right)
$$

Since for positive frequencies, $D^{\prime \prime}(\omega)=\left(\pi D_{0} / 2 \omega_{0}\right) \delta(\omega-$ $\left.\omega_{0}\right)$, the frequency integration is elementary and yields

$$
\Sigma^{\prime \prime}(\omega>0) \propto N\left(\omega-\omega_{0}\right)
$$

We see that the single particle scattering rate is directly proportional to the density of states shifted by the phonon frequency. Clearly, the imaginary part of the fermionic self-energy emerges only when $\omega$ exceeds the threshold

$$
\Omega_{\mathrm{t}} \equiv \Delta+\omega_{0}
$$

the sum of the superconducting gap and the phonon frequency. Right above this threshold, $\tilde{\Sigma}^{\prime \prime}(\omega) \propto(\omega-$ $\left.\Omega_{\mathrm{t}}\right)^{-1 / 2}$. By the Kramers-Kronig relation, this nonanalyticity causes a square root divergence of $\tilde{\Sigma}^{\prime}(\omega)$ at $\omega<\Omega_{\mathrm{t}}$. Combining the two results, we find that near the threshold, $\tilde{\Sigma}(\omega)=A+C / \sqrt{\Omega_{\mathrm{t}}-\omega}$ where $A$ and $C$ are real numbers. By the same reasoning, the pairing vertex $\Phi(\omega)$ also possesses a square-root singularity at $\Omega_{\mathrm{t}}$. Near $\omega=\Omega_{\mathrm{t}}, \Phi(\omega)=B+C / \sqrt{\Omega_{\mathrm{t}}-\omega}$ with real $B$. Since $\Omega_{\mathrm{t}}>\Delta$, we have $A>B$.

The singularity in the fermionic self-energy gives rise to an extra dip-hump structure of the fermionic spectral function at $\mathbf{k}=\mathbf{k}_{F}$. Below $\Omega_{\mathrm{t}}$, the spectral function is zero except for $\omega=\Delta$, where it has a $\delta$-functional peak. Immediately above $\Omega_{\mathrm{t}}, A(\omega) \propto \operatorname{Im}\left(\tilde{\Sigma}(\omega) /\left(\tilde{\Sigma}^{2}(\omega)-\right.\right.$ $\left.\left.\Phi^{2}(\omega)\right)\right)$ takes the form $A(\omega) \propto\left(\omega-\Omega_{\mathrm{t}}\right)^{1 / 2}$. At larger frequencies, $A(\omega)$ passes through a maximum, and eventually vanishes. Adding a small damping introduced by either impurities or finite temperatures, one obtains the spectral function with a peak at $\omega=\Delta$, a dip at $\omega \approx \Omega_{\mathrm{t}}$, and a hump at a somewhat larger frequency. This behavior is shown schematically in Fig. 17.

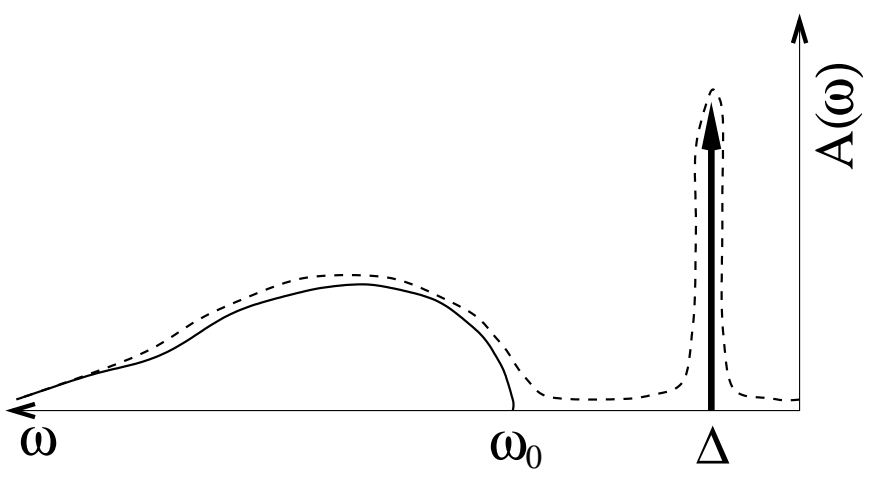

FIG. 17. The schematic form of the quasiparticle spectral function in an $s$-wave superconductor. Solid line $T=0$, dashed line - at finite T. $\Omega_{\mathrm{t}}=\Delta+\omega_{0}$ (from Ref. 149).

The singularities in $\tilde{\Sigma}(\omega)$ and $\Phi(\omega)$ affect other observables such as the fermionic DOS, optical conductivity, Raman response, and the SIS tunneling dynamical conductance 165.68.

For a more complex phonon propagator, which depends on both frequency and momentum, the singularities in the fermionic self-energy and other observables are weaker and may only show up in the derivatives over frequency 167. Still, the opening of the new relaxational channel at $\Omega_{\mathrm{t}}$ gives rise to singularities in the electronic properties of a phonon-mediated $s$-wave superconductor.

\section{B. Similarities and discrepancies between $d$ - and $s$-wave superconductors}

As we already discussed, for magnetically mediated $d$-wave superconductivity, spin fluctuations play the role of phonons. Below $T_{c}$, spin excitations are propagating, magnon-like modes with the gap $\Delta_{s}$. This $\Delta_{s}$ obviously plays the same role as $\omega_{0}$ for phonons, and hence we expect that for spin-mediated pairing, the spectral function should display a peak-dip-hump structure as well. Furthermore, we will demonstrate below that for observables 
such as the DOS, Raman intensity and the optical conductivity, which measure the response averaged over the Fermi surface, the angular dependence of the $d$-wave gap $\Delta(\theta) \propto \cos (2 \theta)$ softens the singularities, but does not wash them out over a finite frequency range. Indeed, we will find that the positions of the singularities are not determined by some averaged gap amplitude but by the maximum value of the $d$-wave gap, $\Delta_{\max }=\Delta$, i.e., the Holstein effect is still present for a $d$-wave superconductor.

Despite many similarities, the feedback effects for phonon-mediated $s$-wave superconductors, and magnetically mediated $d$-wave superconductors are not equivalent as we now demonstrate. The point is that for $s$-wave superconductors, the exchange process shown in Fig.16a is not the only possible source for the fermionic decay: there exists another process, shown in Fig. $16 \mathrm{~b}$, in which a fermion decays into three other fermions. This process is due to a residual four-fermion interaction 165,168 . One can easily make sure that this second process also gives rise to the fermionic decay when the external $\omega$ exceeds a minimum energy of $3 \Delta$, necessary to pull all three intermediate particles out of the condensate of Cooper pairs. At the threshold, the fermionic spectral function is nonanalytic, much like that found at $\Delta+\omega_{0}$. This implies that in $s$-wave superconductors, there are two physically distinct singularities, at $\Delta+\omega_{0}$ and at $3 \Delta$, which come from different processes and therefore are independent of each other. Which of the two threshold frequencies is larger depends on the strength of the coupling and on the shape of the phonon density of states. At weak coupling, $\omega_{0}$ is exponentially larger than $\Delta$, hence the $3 \Delta$ threshold comes first. At strong coupling, $\omega_{0}$ and $\Delta$ are comparable, but calculations within the Eliashberg formalism show that for reałmaterials (e.g. for lead or niobium), still $3 \Delta<\Delta+\omega_{0} \cdot 124$. This result is fully consistent with the photoemission data for these materials 159 .

For magnetically mediated $d$-wave superconductors the situation is different. As we discussed in Section 2, in the one-band model for cuprates, which we adopt, the underlying interaction is a Hubbard-type four-fermion interaction. The introduction of a spin fluctuation as an extra degree of freedom is just a way to account for the fact that there exists a particular interaction channel, where the effective interaction between fermions is the strongest due to the proximity to a magnetic instability. This implies that the spin fluctuation propagator is made out of particle-hole bubbles like those in Fig.16b. Then, to the lowest order in the interaction, the fermionic self-energy is given by the diagram in Fig.16b. Higher-order terms convert a particle-hole bubble in Fig. $16 \mathrm{~b}$. into a wiggly line, and transform this diagram into the one in Fig.16a. Clearly then, inclusion of both diagrams would be double counting, i.e., there is only a single process which gives rise to the threshold in the fermionic self-energy. Note also that the fact that the diagram in Fig $16 \mathrm{~b}$ is a part of that in Fig.16a implies that the development of a singularity in the spectral function at a frequency different from $3 \Delta$ cannot be due to effects outside the spin-fermion model. Indeed, we will show that the model generates two singularities: at $3 \Delta$, and at $\Delta+\Delta_{s}<3 \Delta$. The fact that this is an internal effect, however, implies that $\Delta_{s}$ depends on $\Delta$. The experimental verification of this dependence can then be considered as a "fingerprint" of the spin-fluctuation mechanism. Furthermore, as the singularities at $3 \Delta$ and $\Delta+\Delta_{s}$ are due to the same interaction, their relative intensity is another gauge of the magnetic mechanism for the pairing. We will argue below that some experiments on cuprates particularly measurements of the optical conductivity 160 , allow one to detect both singularities, and that their calculated relative intensity is consistent with the data.

We now discuss separately the behavior of the electronic spectral function, the density of states, SIS tunneling, the Raman intensity and the optical conductivity. To account for all features associated with $d$-wave pairing, we will keep the momentum dependence of the fermionic self-energy and the pairing vertex on momenta along the Fermi surface, although this dependence is indeed weak near hot spots. For simplicity, we assume a circular Fermi surface. In this situation, the $k$-dependence of the self-energy and the pairing vertex reduces to the angular dependence, i.e. $\Sigma=\Sigma(\theta, \omega)$ and $\Phi=\Phi(\theta, \omega)$

\section{The spectral function}

We first consider the spectral function $A_{\mathbf{k}}(\omega)=$ $(1 / \pi)\left|G_{\mathbf{k}}^{\prime \prime}(\omega)\right|$. In the superconducting state, for quasiparticles near the Fermi surface

$$
A_{\mathbf{k}}(\omega>0)=\frac{1}{\pi} \operatorname{Im}\left[\frac{\omega+\Sigma(\theta, \omega)+\varepsilon_{\mathbf{k}}}{(\omega+\Sigma(\theta, \omega))^{2}-\Phi^{2}(\theta, \omega)-\varepsilon_{\mathbf{k}}^{2}}\right] .
$$

By definition, $A_{\mathbf{k}}(-\omega)=A_{\mathbf{k}}(\omega)$.

In a Fermi gas with $d$-wave pairing, $\Sigma(\theta, \omega)=0$, and $\Phi(\theta, \omega)=\Delta(\theta) \propto \cos (2 \theta)$. The spectral function then has a $\delta$-function peak at $\omega=\left(\Delta^{2}(\theta)+\varepsilon_{\mathbf{k}}^{2}\right)^{1 / 2}$. It is obvious, but essential for comparison with the strong coupling case, that the peak disperses with $\mathbf{k}$ and that far away from the Fermi surface one recovers normal state dispersion.

For strong coupling we consider the spectral function, $A_{\mathbf{k}}(\omega)$, for fermions located near hot spots, $\theta=\theta_{h s}$. where the gap $\Delta(\theta)$ ( defined as a solution of $\tilde{\Sigma}^{\prime}(\omega=$ $\left.\left.\Delta, \theta_{h s}\right)=\Phi^{\prime}\left(\omega=\Delta, \theta_{h s}\right)\right)$ is maximum. As discussed above, we expect the spectral function to possess a peak at $\omega=\Delta$ and a singularity at $\omega=\Omega_{\mathrm{t}}=\Delta+\Delta_{s}$. The behavior of $A(\omega)$ near the singularity is robust and can be obtained without a precise knowledge of the frequency dependence of $\tilde{\Sigma}(\omega)$ and $\Phi(\omega)$. All we need to know is that near $\omega=\Delta, \tilde{\Sigma}^{2}(\omega)-\Phi^{2}(\omega) \propto \omega-\Delta$. Substituting this form into Eq.70 and converting to the real axis using the spectral representation, we obtain for $\omega=\Omega_{\mathrm{t}}+\epsilon$ 


$$
\tilde{\Sigma}^{\prime \prime}(\omega) \propto \int_{0}^{\epsilon} \frac{d x}{(x(\epsilon-x))^{1 / 2}}
$$

This integral is the same as in Eq.76, hence $\tilde{\Sigma}^{\prime \prime}$ undergoes a finite jump at $\omega=\Omega_{\mathrm{t}}$, just as the spin polarization operator does at $\omega=2 \Delta$. By the Kramers-Kronig relation, this jump gives rise to a logarithmic divergence of $\tilde{\Sigma}^{\prime}$. The same singular behavior holds for the pairing vertex $\Phi(\omega)$, with exactly the same prefactor in front of the logarithm. The last result implies that $\tilde{\Sigma}(\omega)-\Phi(\omega)$ is non-singular at $\omega=\Omega_{\mathrm{t}}$. Substituting these results into Eq.84, we find that the spectral function $A(\omega)$ behaves at $\omega>\Omega_{\mathrm{t}}$ as $1 / \log ^{2}\left(\omega-\Omega_{\mathrm{t}}\right)$, i.e., almost discontinuously. Obviously, at a small but finite $T$, the spectral function should have a dip very near $\omega=\Omega_{\mathrm{t}}$, and a hump at a somewhat higher frequency.

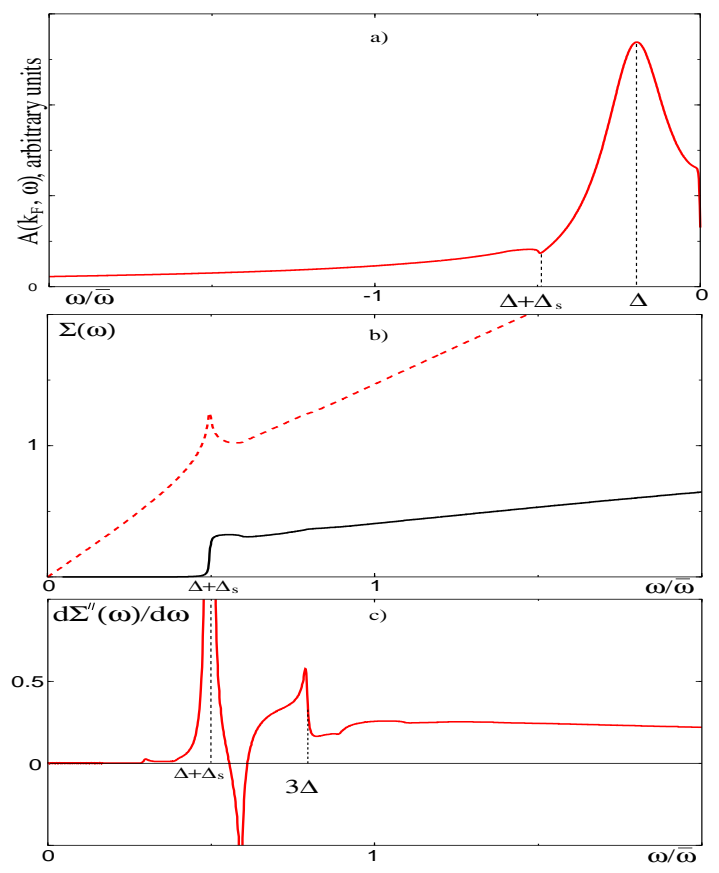

FIG. 18. Upper panel: the quasiparticle spectral function determined by solving the coupled Eliashberg equations for $\lambda=1$. The peak-dip-hump structure of $A(\omega)$ is clearly visible but not dramatic. Middle panel: real and imaginary parts of the fermionic self-energy (dashed and solid lines, respectively) . Lower panel -the frequency derivative of $\Sigma^{\prime \prime}(\omega)$. The extra structure at $3 \Delta$ is clearly visible. The figure is taken from Ref. 149 .

In Fig. 18 we present the result for $A(\omega)$ obtained from a solution of the set of three coupled Eliashberg equations at $T \ll T_{c} 149$. This solution is consistent with our analytical estimate. We clearly see that the fermionic spectral function has a peak-dip-hump structure, and the peak-dip distance equals $\Delta_{s}$. We also see in Fig 18 that the fermionic self-energy is non-analytic at $\omega=3 \Delta$. As we discussed above, this last non-analyticity originates in the non-analyticity of the dynamical spin susceptibility at $\omega=2 \Delta$.
Another "fingerprint" of the spin-fluctuation scattering can be found by studying the evolution of the spectral function as one moves away from the Fermi surface. The argument here goes as follows: at strong coupling, where $\Delta \geq \omega_{\text {sf }}$, probing the fermionic spectral function at frequencies progressively larger than $\Delta$, one eventually probes the normal state fermionic self-energy at $\omega \gg \omega_{\text {sf. }}$. Substituting the self energy Eq.47 into the fermionic propagator, we find that up to $\omega \sim \bar{\omega}$, the spectral function in the normal state does not have a quasiparticle peak at $\omega=\varepsilon_{\mathbf{k}}$. Instead, it only displays a broad maximum at $\omega=\varepsilon_{\mathbf{k}}^{2} / \bar{\omega}$. In other words, at $\omega_{\text {sf }}<\omega<\bar{\omega}$, the spectral function in the normal state displays non-Fermi liquid behavior with no quasiparticle peak (see Fig. 9). The absence of a quasiparticle peak in the normal state implies that the sharp quasiparticle peak that we found at $\omega=\Delta$ for momenta at the Fermi surface cannot simply disperse with $\mathbf{k}$, as it does for noninteracting fermions with a $d$-wave gap. Specifically, the quasiparticle peak cannot move further in energy than $\Delta+\Delta_{s}$ since at larger frequencies, spin scattering rapidly increases, and the fermionic spectral function should display roughly the same non-Fermi-liquid behavior as in the normal state.

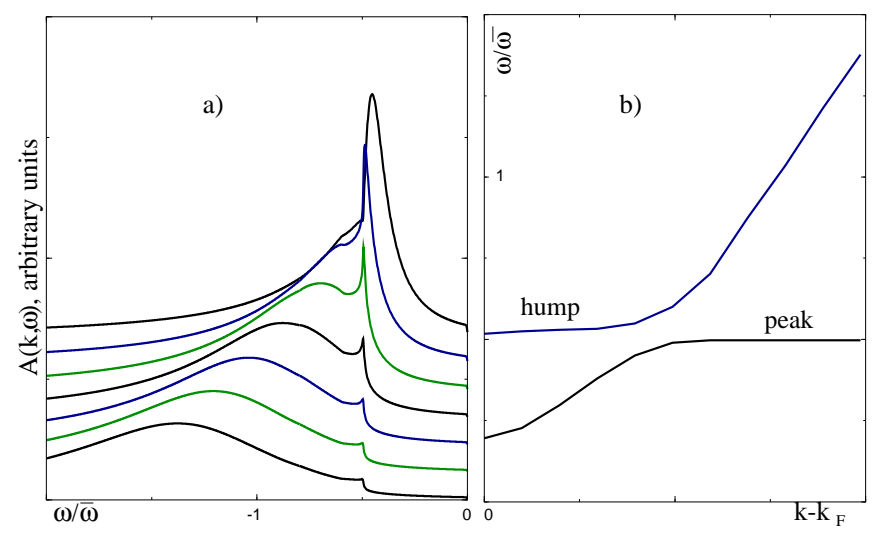

FIG. 19. a) Frequency dependence of the spectral function in the superconducting state for different $\epsilon_{k}$. The curve at the bottom has a highest $\epsilon_{k}$. No coherent quasiparticle peak occurs for energies larger than $\Delta+\Delta_{s}$. Instead, the spectral function displays a broad maximum, similar to that in the normal state. (From Ref 149.)

In Fig 19 a we present plots for the spectral function as the momentum moves away from the Fermi surface. We see the behavior we just described: the quasiparticle peak does not move further than $\Delta+\Delta_{s}$. Instead, when $\mathbf{k}-\mathbf{k}_{\mathrm{F}}$ increases, it gets pinned at $\Delta+\Delta_{s}$ and gradually looses its spectral weight. At the same time, the hump disperses with $\mathbf{k}$ and for frequencies larger than $\Delta+\Delta_{s}$ gradually transforms into a broad maximum at $\omega=\varepsilon_{\mathbf{k}}^{2} / \bar{\omega}$. The positions of the peak and the dip versus $\mathbf{k}-\mathbf{k}_{\mathrm{F}}$ are presented in Fig. $19 \mathrm{~b}$. 


\section{The density of states}

The quasiparticle density of states, $N(\omega)$, is the momentum integral of the spectral function:

$$
N(\omega)=\int \frac{d^{2} \mathbf{k}}{4 \pi^{2}} A_{\mathbf{k}}(\omega) .
$$

Substituting $A_{\mathbf{k}}(\omega)$ from Eq. 84 and integrating over $\varepsilon_{\mathbf{k}}$, one obtains

$$
N(\omega) \propto \operatorname{Im} \int_{0}^{2 \pi} \mathrm{d} \theta \frac{\omega+\Sigma(\theta, \omega)}{\left(\Phi^{2}(\theta, \omega)-(\omega+\Sigma(\theta, \omega))^{2}\right)^{1 / 2}},
$$

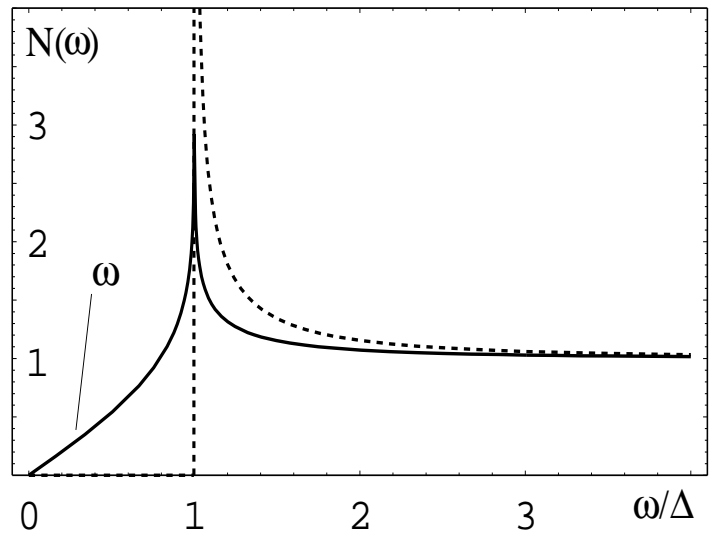

FIG. 20. Density of states of a noninteracting Fermi gas with $d$-wave gap (golid line) and with $s$-wave gap (dashed line). (From Ref.149).

We first consider $N(\omega)$ in a $d$-wave gas, and then discuss strong coupling effects. In a $d$-wave gas, $\Sigma=0$ and $\Delta_{\mathbf{k}}=\Delta \cos (2 \theta)$. Integrating in Eq.87 over $\theta$ we obtain 120.173

$$
\begin{aligned}
N(\omega) & =N_{0} \operatorname{Re}\left[\frac{\omega}{2 \pi} \int_{0}^{2 \pi} \frac{\mathrm{d} \theta}{\sqrt{\omega^{2}-\Delta^{2} \cos ^{2}(2 \theta)}}\right] \\
& =\frac{2 N_{0}}{\pi} \begin{cases}K(\Delta / \omega) & \text { for } \omega>\Delta ; \\
(\omega / \Delta) K(\omega / \Delta) & \text { for } \omega<\Delta .\end{cases}
\end{aligned}
$$

where $K(x)$ is the elliptic integral of first kind. We see that $N(\omega) \sim \omega$ for $\omega \ll \Delta$ and diverges logarithmic as $(1 / \pi) \ln (8 \Delta /|\Delta-\omega|)$ for $\omega \approx \Delta$. At larger frequencies, $N(\omega)$ gradually decreases towards the frequency independent, normal state value of the DOS, which we have normalized to unity. The plot of $N(\omega)$ in a $d$-wave BCS superconductor is presented in Fig. 20 .

For comparison, in an $s$-wave superconductor, the DOS vanishes at $\omega<\Delta$ and diverges as $N(\omega) \propto(\omega-\Delta)^{-1 / 2}$ at $\omega \geq \Delta$. We see that a $d$-wave superconductor is different in that (i) the DOS is finite down to the smallest frequencies, and (ii) the singularity at $\omega=\Delta$ is weaker (logarithmic). Still, however, $N(\omega)$ is singular only at a frequency which equals to the largest value of the $d$-wave gap. This illustrates a point made earlier: the angular dependence of the $d$-wave gap reduces the strength of the singularity at $\omega=\Delta_{\max }(\theta)$, but does not wash it out over a finite frequency range.
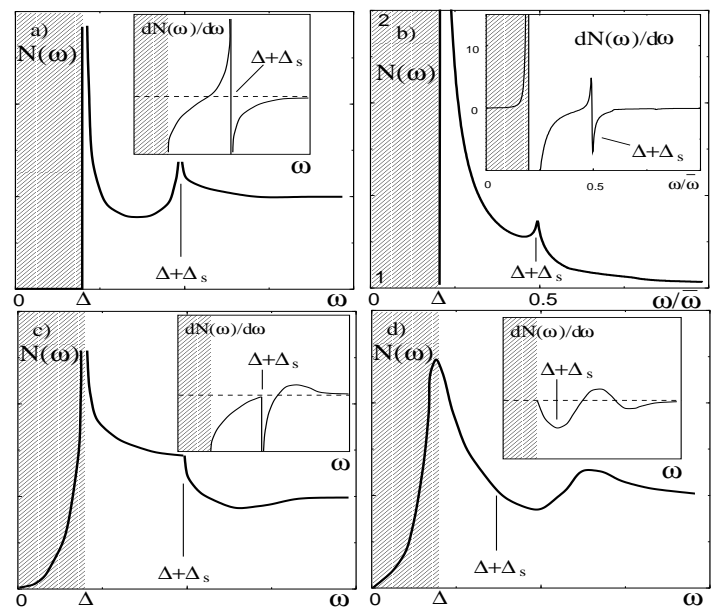

FIG. 21. (a) The behavior of the SIN tunneling conductance (i.e., DOS) in a strongly coupled $d$-wave superconductor. Main pictures - $N(\omega)$, insets - $d N(\omega) / d \omega$. (a) The schematic behavior of the DOS for a flat gap. (b) The solution of the Eliashberg-type equations for a flat gap. The shaded regions are the ones in which the flat gap approximation is incorrect as the physics is dominated by nodal quasiparticles. (c) The schematic behavior of $N(\omega)$ for the quadratic variation of the gap near its maxima. (d) The expected behavior of the DOS in a real situation when singularities are softened out by finite $T$ or impurity scattering. The position of $\Delta+\Delta_{s}$ roughly corresponds to a minimum of $d N(\omega) / d \omega$. The figure is taken from Ref. 149 .

We now turn to strong coupling. We first demonstrate that the DOS possesses extra peak-dip features, associated with the singularities in $\tilde{\Sigma}(\omega)$ and $\Phi(\omega)$ at $\omega=\Omega_{\mathrm{t}}$ which for spin-mediated pairing is

$$
\Omega_{\mathrm{t}}=\Delta+\Delta_{s}
$$

An analytical approach proceeds as follows 172 . Consider first a case when the gap is totally flat near a hot spot. At $\omega=\Omega_{\mathrm{t}}$, both $\tilde{\Sigma}(\omega)$ and $\Phi(\omega)$ diverge logarithmically. On substituting these forms into Eq. 87, we find that $N(\omega)$ has a logarithmic singularity:

$$
N_{\text {sing }}(\omega) \propto\left(\log \frac{1}{\left|\omega-\Omega_{\mathrm{t}}\right|}\right)^{1 / 2} .
$$

This singularity gives rise to a strong divergence of $d N(\omega) / d \omega$ at $\omega=\Omega_{\mathrm{t}}$, a behavior schematically shown in Fig. 21a). In part (b) of this figure we present the result for $N(\omega)$ obtained by the solution of the Eliashberg-type Eqs.66 72. A small but finite temperature was used to smear out divergences. We recall that the Eliashberg set does not include the angular dependence of the gap near 
hot spots, and hence the numerical result for the DOS in Fig. 21b should be compared with Fig. 21a. We clearly see that $N(\omega)$ has a second peak at $\omega=\Omega_{\mathrm{t}}$. This peak strongly affects the frequency derivative of $N(\omega)$ which become singular near $\Omega_{\mathrm{t}}$.

The relatively small magnitude of the singularity in $N(\omega)$ is a consequence of the linearization of the fermionic dispersion near the Fermi-surface. For an actual $\epsilon_{\mathbf{k}}$ chosen to fit ARPES data 128 , nonlinearities in the fermionic dispersion occur at energies comparable to $\Omega_{\mathrm{t}}$. This is due to the fact that hot spots are located close to $(0, \pi)$ and related points at which the Fermi velocity vanishes. As a consequence, the momentum integration in the spectral function should have a less pronounced smearing effect than found in our calculations, and the frequency dependence of $N(\omega)$ should more resemble that of $A(\omega)$ for momenta where the gap is at maximum.

For a momentum dependent gap, the behavior of fermions near hot spots is the same as when the gap is flat, but now $\Omega_{\mathrm{t}}$ depends on $\theta$ as both $\Delta$ and $\Delta_{s}$ vary as one moves away from a hot spot. The variation of $\Delta$ is obvious, the variation of $\Delta_{s}$ is due to the fact that this frequency scales as $\Delta^{1 / 2}$. Since both $\Delta$ and $\Delta_{s}$ are maximal at a hot spot, we can model the momentum dependence by replacing

$$
\Omega_{\mathrm{t}} \rightarrow \Omega_{\mathrm{t}}-a \tilde{\theta}^{2} .
$$

where $\tilde{\theta}=\theta-\theta_{h s}$, and $a>0$. The singular pieces of the self-energy and the pairing vertex then behave as $\log \left(\Omega_{\mathrm{t}}-\omega-a \widetilde{\theta}^{2}\right)^{-1}$. Substituting these forms into Eq.87 and using the fact that $\tilde{\Sigma}(\omega)-\Phi(\omega) \approx$ const at $\omega \approx \Omega_{\mathrm{t}}$, we obtain

$$
N_{\text {sing }}(\omega) \propto \operatorname{Re} \int \mathrm{d} \widetilde{\theta}\left[\log \left(\Omega_{\mathrm{t}}-\omega-\mathrm{a} \widetilde{\theta}^{2}\right)^{-1}\right]^{-1 / 2} .
$$

A straightforward analysis of the integral shows that now $N(\omega)$ displays a one-sided non-analyticity at $\omega=\Omega_{\mathrm{t}}$ :

$$
N_{\text {sing }}(\omega)=-B \Theta\left(\omega-\Omega_{\mathrm{t}}\right)\left(\frac{\omega-\Omega_{\mathrm{t}}}{\left|\log \left(\omega-\Omega_{\mathrm{t}}\right)\right|}\right)^{1 / 2},
$$

where $B>0$, and $\Theta(x)=1$ for $x>0$, and $\Theta(x)=0$ for $x<0$. This non-analyticity gives rise to a cusp in $N(\omega)$ right above $\Omega_{t}$, and one-sided square-root divergence of the frequency derivative of the DOS. This behavior is shown schematically in Fig. 21c. Comparing this behavior with that shown in Fig. 21a for a flat gap, we observe that the angular dependence of the gap predominantly affects the form of $N(\omega)$ at $\omega \leq \Omega_{\mathrm{t}}$. At these frequencies, the angular variation of the gap completely eliminates the singularity in $N(\omega)$. At the same time, above $\Omega_{\mathrm{t}}$, the angular dependence of the gap softens the singularity, but, still, the DOS sharply drops above $\Omega_{\mathrm{t}}$ in such a way that the derivative of the DOS diverges on approaching $\Omega_{\mathrm{t}}$ from above. We see again that in a $d$-wave superconductor, the singularity in the DOS is softened by the angular dependence of the gap, but still persists at a particular frequency related to the maximum value of the gap. This point is essential as it enables us to read off the maximum gap value directly from the experimental data without any "deconvolution" of momentum averages.

For real materials, in which singularities are removed by e.g., impurity scattering, $N(\omega)$ likely has a dip at $\omega \geq \Omega_{\mathrm{t}}$ and a hump at a larger frequency. This is schematically shown in Fig. (21)d. The location of $\Omega_{\mathrm{t}}$ is best described as a point where the frequency derivative of the DOS passes through a minimum. The singularity in $d N(\omega) / d \omega$ at $\Omega_{\mathrm{t}}$, and the dip-hump structure of $N(\omega)$ at $\omega \geq \Omega_{\mathrm{t}}$ are additional "fingerprints" of the spinfluctuation mechanism in the single particle response.

\section{E. SIS tunneling}

Measurements of the dynamical conductance $d I / d V$ through a superconductor - insulator - superconductor (SIS) junction offer another tool to search for the fingerprints of the spin-fluctuation mechanism. The conductance through this junction is the derizative over voltage of the convolution of the two DOS124: $d I / d V \propto S(\omega)$ where

$$
S(\omega)=N_{0}^{-2} \int_{0}^{\omega} d \Omega N(\omega-\Omega) \partial_{\Omega} N(\Omega) .
$$

The DOS in a $d$-wave gas is given in Eq. 88. Substituting this form into Eq. 94 and integrating over frequency we obtain the result presented in Fig.22. At small $\omega, S(\omega)$ is quadratic in $\omega 120$. This is an obvious consequence of the fact that the DOS is linear in $\omega$. At $\omega=2 \Delta, S(\omega)$ undergoes a finite jump. This jump is related to the fact that near $2 \Delta$, the integral over the two DOS includes the region around $\Omega=\Delta$ where both $N(\Omega)$ and $N(\omega-\Omega)$ are logarithmically singular, and $\partial_{\Omega} N(\Omega)$ diverges as $1 /(\Omega-\Delta)$. The singular contribution to $S(\omega)$ from this region can be evaluated analytically and yields

$$
S(\omega)=-\frac{1}{\pi^{2}} P \int_{-\infty}^{\infty} \frac{d x \log |x|}{x+\omega-2 \Delta}=-\frac{1}{2} \operatorname{sign}(\omega-2 \Delta)
$$

Observe that the amplitude of the jump in the SIS conductance is a universal number which does not depend on the value of $\Delta$. At larger frequencies, $S(\omega)$ continuously goes down and eventually approaches a value of $S(\omega \rightarrow \infty)=1$. 

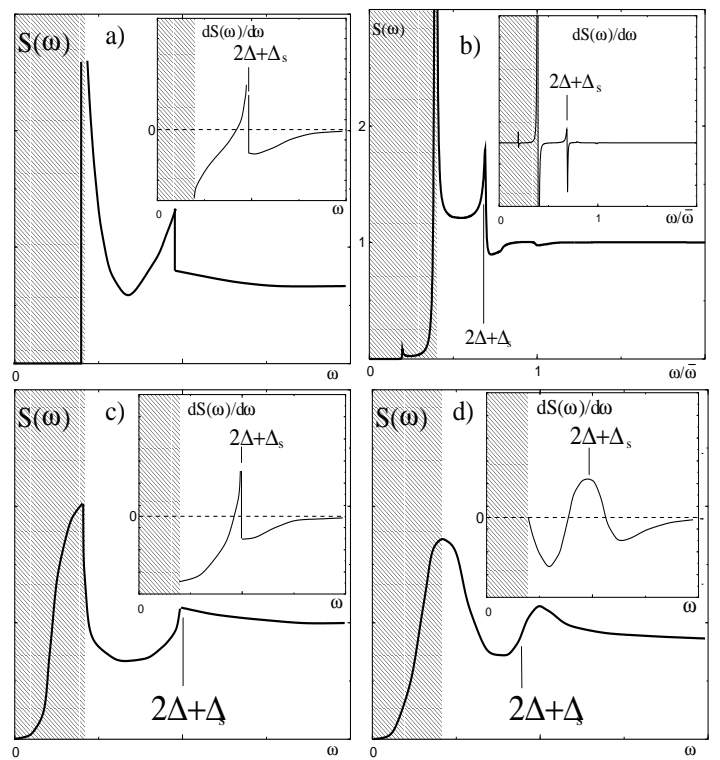

FIG. 22. (a) The schematic behavior of the SIS tunneling conductance, $S(\omega)$, in a strongly coupled $d$-wave superconductor. Main pictures - $S(\omega)$, insets - $d S(\omega) / d \omega$. (a) The schematic behavior of $S(\omega)$ for a flat gap. (b) The solution of the Eliashberg-type equations for a flat gap using the DOS from the previous subsection. The shaded regions are the ones in which the flat gap approximation is incorrect as the physics is dominated by nodal quasiparticles. (c) The schematic behavior of $S(\omega)$ for a quadratic variation of the gap near its maxima. (d) The expected behavior of the SIS conductance in a real situation when singularities are softened out by finite $T$ or by impurity scattering. $2 \Delta+\Delta_{s}$ roughly corresponds to the maximum of $d S(\omega) / d \omega$. (From Ref. 149).

In the case of strong coupling one finds again that the quadratic behavior at low frequencies and the discontinuity at $2 \Delta$ survive at arbitrary coupling. Indeed, the quadratic behavior at low $\omega$ is just a consequence of the linearity of $N(\omega)$ at low frequencies. Therefore, just as we did for the density of states we concentrate on behavior above $2 \Delta$ that is sensitive to strong coupling effects.

Consider first how the singularity in $\tilde{\Sigma}(\omega)$ at $\Omega_{\mathrm{t}}$ affects $S(\omega)$. From a physical perspective, we would expect a singularity in $S(\omega)$ at $\omega=\Delta+\Omega_{\mathrm{t}}=2 \Delta+\Delta_{s}$. Indeed, to get a nonzero SIS conductance, one has to first break a Cooper pair, which costs an energy of $2 \Delta$. After a pair is broken, one of the electrons becomes a quasiparticle in a superconductor and takes the energy $\Delta$, while the other tunnels. If the tunneling voltage equals $\Delta+\Omega_{\mathrm{t}}$, the electron which tunnels through a barrier has energy $\Omega_{\mathrm{t}}$, and can emit a spin excitation and fall to the bottom of the band (see Fig. 23). This behavior is responsible for the drop in $d I / d V$ and is schematically shown in Fig. 22.

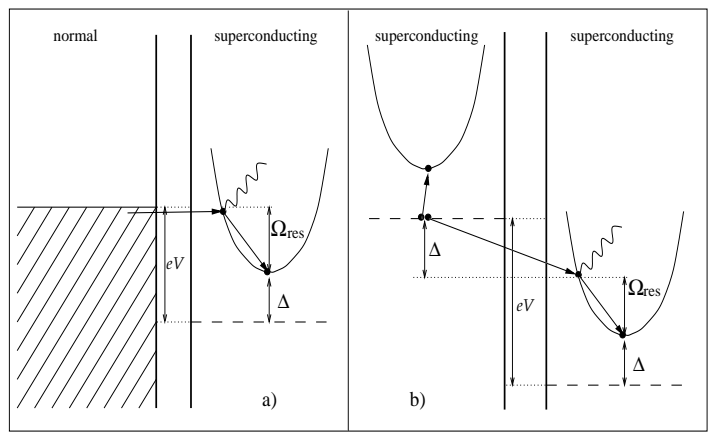

FIG. 23. The schematic diagram for the dip features in SIN and SIS tunneling conductances (figures a and b, respectively). For SIN tunneling, which measures the fermionic DOS, the electron which tunnels from a normal metal can emit a propagating magnon if the voltage $e V=\Delta+\Delta_{s}$. After emitting a magnon, the electron falls to the bottom of the band. This leads to a sharp reduction of the current and produces a drop in $d I / d V$. For SIS tunneling, the physics is similar, but one first has to break an electron pair, which costs energy $2 \Delta$ (taken from Ref. 172).

Consider this effect in more detail 172 149. We first note that $\omega=\Delta+\Omega_{\mathrm{t}}$ is special for Eq. 94 because both $d N(\Omega) / d \Omega$ and $N(\omega-\Omega)$ diverge at the same energy, $\Omega=\Omega_{\mathrm{t}}$. Substituting the general forms of $N(\omega)$ near $\omega=\Omega_{\mathrm{t}}$ and $\omega=\Delta$, we obtain after simple manipulations that for a flat gap, $S(\omega)$ has a one-sided divergence at $\omega=\Omega_{\mathrm{t}}+\Delta=2 \Delta+\Delta_{s}$.

$$
S_{\text {sing }}(\epsilon) \propto \frac{\Theta(-\epsilon)}{\sqrt{-\epsilon}}
$$

where $\epsilon=\omega-\left(\Omega_{\mathrm{t}}+\Delta\right)$. This obviously causes a divergence of the frequency derivative of $S(\omega)$ (i.e., of $\left.d^{2} I / d V^{2}\right)$. This behavior is schematically shown in Fig. $22 \mathrm{a}$. In Fig. $22 \mathrm{~b}$ we present the results for $S(\omega)$ obtained by integrating theoretical $N(\omega)$ from Fig.21b. We clearly see that $S(\omega)$ and its frequency derivative are singular at $\omega=2 \Delta+\Delta_{s}$, in agreement with the analytical prediction.

For a quadratic variation of the gap near the maxima, calculations similar to those for the SIN tunneling yield the result that $S(\omega)$ is continuous through $2 \Delta+\Delta_{s}$, but its frequency derivative diverges as

$$
\frac{d S(\omega)}{d \omega} \propto P \int_{0}^{\Delta} \frac{d x}{(x|\log x|)^{1 / 2}(x-\epsilon)} \sim \frac{\Theta(-\epsilon)}{\left.|\epsilon \log | \epsilon\right|^{1 / 2}} .
$$

The singularity in the derivative implies that near $\epsilon=0$

$$
S(\epsilon)=S(0)-C \Theta(-\epsilon)\left(\frac{-\epsilon}{|\log (-\epsilon)|}\right)^{1 / 2}
$$

where $C>0$. This behavior is schematically presented in Fig. 22d. We again see that the angular dependence of the gap softens the strength of the singularity, but 
the singularity remains confined to a single frequency $\omega=2 \Delta+\Delta_{s}$.

In real materials, the singularity in $S(\omega)$ is softened and transforms into a dip slightly below $2 \Delta+\Delta_{s}$, and a hump at a frequency larger than $2 \Delta+\Delta_{s}$. The frequency $2 \Delta+\Delta_{s}$ roughly corresponds to a maximum of the frequency derivative of the SIS conductance.

\section{F. Optical conductivity and Raman response}

Further phenomena sensitive to $\Omega_{\mathrm{t}}$ are the optical conductivity, $\sigma(\omega)$ and the Raman response, $R(\omega)$. Both are proportional to the fully renormalized particle-hole polarization bubble, but with different signs attributed to the bubble composed of anomalous propagators. Specifically, after integrating in the particle-hole bubble over $\varepsilon_{\mathbf{k}}$, one obtains

$$
\begin{aligned}
& R(\omega)=\operatorname{Im} \int \mathrm{d} \omega^{\prime} \mathrm{d} \theta \mathrm{V}^{2}(\theta) \Pi_{\mathrm{r}}\left(\theta, \omega, \omega^{\prime}\right) \\
& \sigma(\omega)=\operatorname{Re}\left(\frac{\mathrm{i}}{\omega+\mathrm{i} \delta} \int \mathrm{d} \omega^{\prime} \mathrm{d} \theta \Pi_{\sigma}\left(\theta, \omega, \omega^{\prime}\right)\right),
\end{aligned}
$$

where $V(\theta)$ is a Raman vertex which depends on the scattering geometry 177 , and

$$
\Pi_{r, \sigma}\left(\theta, \omega, \omega^{\prime}\right)=\frac{\tilde{\Sigma}_{+} \tilde{\Sigma}_{-}+\alpha \Phi_{+} \Phi_{-}+D_{+} D_{-}}{D_{+} D_{-}\left(D_{+}+D_{-}\right)}
$$

Here $\alpha=-1$ for $\Pi_{r}$, and $\alpha=1$ for $\Pi_{\sigma}$. Also, $\tilde{\Sigma}_{ \pm}=$ $\tilde{\Sigma}\left(\omega_{ \pm}\right)$and $\Phi_{ \pm}=\Phi\left(\omega_{ \pm}\right)$, where $\omega_{ \pm}=\omega^{\prime} \pm \omega / 2$. We also introduced $D_{ \pm}=\left(\Phi_{ \pm}^{2}-\tilde{\Sigma}_{ \pm}^{2}\right)^{1 / 2}$. Note that $\tilde{\Sigma}$ and $\Phi$ depend on $\omega$ and $\theta$.

In a superconducting gas, the optical conductivity vanishes identically for any nonzero frequency due to the absence of a physical scattering between quasiparticles in a gas. The presence of a superconducting condensate, however, gives rise to a $\delta$ functional term in $\sigma$ at $\omega=0$ : $\sigma(\omega)=\pi \delta(\omega) \int d \theta d \omega^{\prime} \Pi_{\sigma}\left(\theta, 0, \omega^{\prime}\right)$. This behavior is typical for any BCS superconductor 178. The behavior of $\sigma(\omega)$ for a d-wave gas with additional impurities, causing inelastic scattering, is more complex and has been discussed in Ref. 180.

The form of the Raman intensity depends on the scattering geometry. For the scattering in the $B_{1 g}$ channel, the Raman vertex has the same angular dependence as the $d$-wave gap, i.e., $V(\theta) \propto \cos (2 \theta)$ 179. 177 . Straightforward computations then show that at low frequencies, $R(\omega) \propto \omega^{3}$ 179. For a constant $V(\theta)$, we would have $R(\omega) \propto \omega$.

Near $\omega=2 \Delta$, the $B_{1 g}$ Raman intensity is singular. For this frequency, both $D_{+}$and $D_{-}$vanish at $\omega^{\prime}=0$ and $\theta=0$. This causes the integral for $R(\omega)$ to be divergent. The singular contribution to $R(\omega)$ can be obtained analytically by expanding in the integrand to leading order in $\omega^{\prime}$ and in $\theta$. Using the spectral representation, we then obtain, for $\omega=2 \Delta+\delta 177$

$$
\begin{aligned}
R(\omega)= & \int_{0}^{\epsilon} d \Omega \int d \tilde{\theta} \frac{1}{\sqrt{\Omega+a \tilde{\theta}^{2}} \sqrt{\delta-\Omega+a \tilde{\theta}^{2}}} \\
& \times \frac{1}{\left(\sqrt{\Omega+a \tilde{\theta}^{2}}+\sqrt{\delta-\Omega+a \tilde{\theta}^{2}}\right)}
\end{aligned}
$$

where, as before, $\tilde{\theta}=\theta-\theta_{h s}$ For a flat band $(a=0)$, $R(\omega) \propto R e\left[(\omega-2 \Delta)^{-1 / 2}\right]$. For $a \neq 0$, i.e., for a quadratic variation of the gap near its maximum, the $2 \mathrm{~d}$ integration in Eq.101 is elementary, and yields $R(\omega) \propto \log |\omega-2 \Delta|$. At larger frequencies $R(\omega)$ gradually decreases.

The behavior of $R(\omega)$ in a d-wave gas is shown in Fig.24. Observe that due to the interplay of numerical factors, the logarithmic singularity shows up only in the near vicinity of $2 \Delta$, while at somewhat larger $\omega$, the angular dependence of the gap becomes irrelevant, and $R(\omega)$ behaves as $(\omega-2 \Delta)^{1 / 2}$, i.e. the same as for a flat gap 173 .

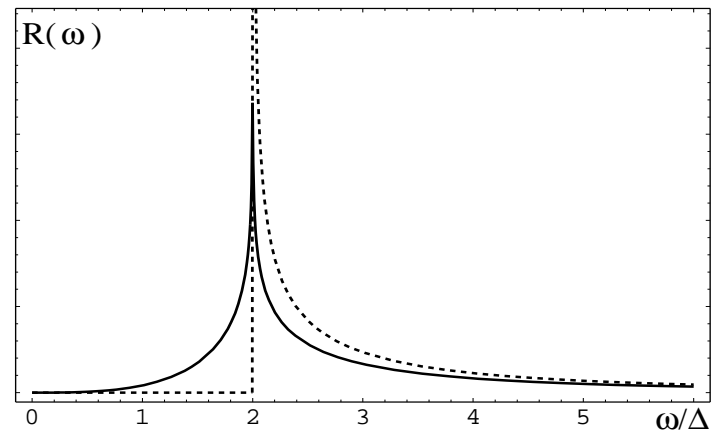

FIG. 24. The behavior of the Raman response in a BCS superconductor with a flat gap (dashed line), and for a quadratic variation of the gap near its maximum (solid line).

We now consider strong coupling effects. A nonzero fermionic self-energy mostly affects the optical conductivity for the simple reason that it becomes finite in the presence of spin scattering which can relax fermionic momenta. For a momentum-independent gap, a finite conductivity emerges above a sharp threshold. This threshold stems from the fact that at least one of the two fermions in the conductivity bubble should have a finite $\tilde{\Sigma}^{\prime \prime}$, i.e., its energy should be larger than $\Omega_{\mathrm{t}}$. Another fermion should be able to propagate, i.e., its energy should be larger than $\Delta$. The combination of the two requirements yields the threshold for $\sigma(\omega>0)$ at $2 \Delta+\Omega_{\mathrm{t}}$, i.e., at the same frequency where the SIS tunneling conductance is singular. One can easily demonstrate that for a flat gap, the conductivity behaves above the threshold as $\epsilon^{1 / 2} / \log ^{2} \epsilon$, where $\epsilon=\omega-\left(\Delta+\Omega_{\mathrm{t}}\right)=\omega-\left(2 \Delta+\Delta_{s}\right)$. This singularity obviously causes a divergence of the first derivative of the conductivity at $\epsilon=+0$.

In Fig.25 we show the result for the conductivity obtained by solving the set of coupled Eliashberg-type equations, Eqs $66-72$ 109, 181. We see the expected singularity at $2 \Delta+\Delta_{s}$. The insert shows the behavior of the inverse conductivity $1 / \sigma(\omega)$ Observe that $1 / \sigma(\omega)$ is linear in $\omega$ over a rather wide frequency range 181 . 


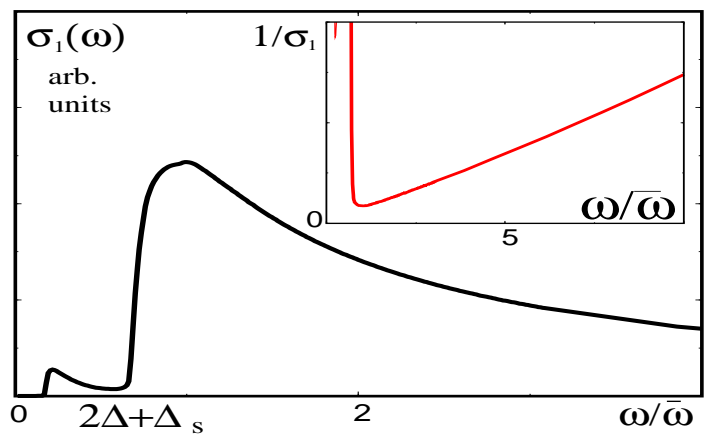

FIG. 25. The real part of the optical conductivity $\sigma_{1}(\omega)$ at the lowest $T$ obtained using the self energy and the pairing vertex from the solution of the Eliashberg equations for $\lambda=1$. The onset of the optical response is $\omega=2 \Delta+\Delta_{s}$. The contributions from nodal regions (not included in calculations) yield a nonzero conductivity at all $\omega$. Inset - the behavior of the inverse conductivity vs frequency. (From Ref. 109).

For a true $d$-wave gap, the conductivity is finite for all frequencies simply because the angular integration in Eq. 99 involves the region near the nodes, where $\tilde{\Sigma}^{\prime \prime}$ is nonzero down to the lowest frequencies. Still, the conductivity is singular at $\Omega_{\mathrm{t}}+\Delta$ as we now demonstrate. Indeed, as we already discussed, at deviations from $\theta=\theta_{h s}$, where the gap is at maximum, both $\Delta$ and $\Delta_{s}$ decrease, hence $\Omega_{\mathrm{t}}(\theta)=\Omega_{\mathrm{t}}-a \tilde{\theta}^{2}$, where $\tilde{\theta}=\theta-\theta_{h s}$ and $a>0$. The singular pieces in $\tilde{\Sigma}(\omega)$ and $\Phi(\omega)$ then behave as $\left|\log \left(\Omega_{\mathrm{t}}-\omega-a \theta^{2}\right)\right|$. Substituting these forms into the particle-hole bubble and integrating over $\theta$, we find that the conductivity and its first derivative are continuous at $\omega=2 \Delta+\Delta_{s}$, but the second derivative of the conductivity diverges as $d^{2} \sigma / d \omega^{2} \propto 1 /\left(|\epsilon| \log ^{2} \epsilon\right)$. We see that the singularity is weakened by the angular dependence of the gap, but is still located exactly at $\Omega_{\mathrm{t}}+\Delta=2 \Delta+\Delta_{s}$.

The same reasoning can be applied to a region near $4 \Delta$. The singularity at $4 \Delta$ is also weakened by the angular dependence of the gap, but is not shifted and still should show up in the second derivative of the conductivity.

For the Raman intensity, strong coupling effects are less relevant. First, one can prove along the same lines as in previous subsections that the cubic behavior at low frequencies for $B_{1 g}$ scattering (and the linear behavior for angular independent vertices), and the logarithmic singularity at $2 \Delta$, are general properties of a $d$-wave superconductor, which survive for all couplings. Thus, analogous to the density of states and the SIS-tunneling spectrum, the Raman response below $2 \Delta$ is not sensitive to strong coupling effects. Second, near $\omega_{0}+\Delta$, singular contributions which come from $\tilde{\Sigma}_{+} \tilde{\Sigma}_{-}$and $\Phi_{+} \Phi_{-}$ terms in $\Pi_{r}$ in Eq. 99 cancel each other. As a result, for a flat gap, only the second derivative of $R(\omega)$ diverges at $\Delta+\Omega_{\mathrm{t}}$. For a quadratic variation of a gap near its maximum, the singularity is even weaker and shows up only in the third derivative of $R(\omega)$. Obviously, this is a very weak effect, and its experimental determination is difficult.
We now argue that measurements of the optical conductivity allow one not only to verify the magnetic scenario, but also to determine both $\Delta_{s}$ and $\Delta$ independently in the same experiment. In the magnetic scenario, the fermionic self-energy is singular at two frequencies: at $\Omega_{\mathrm{t}}=\Delta+\Delta_{s}$, which is the onset frequency for spinfluctuation scattering near hot spots, and at $\omega=3 \Delta$, where fermionic damping near hot spots first emerges due to a direct four-fermion interaction. Since in the spin-fluctuation mechanism, both singularities are due to the same underlying interaction, their relative intensity can be obtained within the model.

In general, the singularity at $3 \Delta$ is much weaker at strong coupling, and can be detected only in the analysis of the derivatives of the fermionic self-energy. We recall that the singularity in $\tilde{\Sigma}(\omega)$ at $\Omega_{\mathrm{t}}$ gives rise to singularity in the conductivity at $\Delta+\Omega_{\mathrm{t}}$, while the $3 \Delta$ singularity in $\tilde{\Sigma}(\omega)$ obviously causes a singularity in conductivity at $\omega=4 \Delta$. In addition, we expect a singularity in $\sigma(\omega)$ at $2 \Omega_{\mathrm{t}}$, as at this frequency both fermions in the bubble have a singular $\tilde{\Sigma}\left(\Omega_{\mathrm{t}}\right)$.

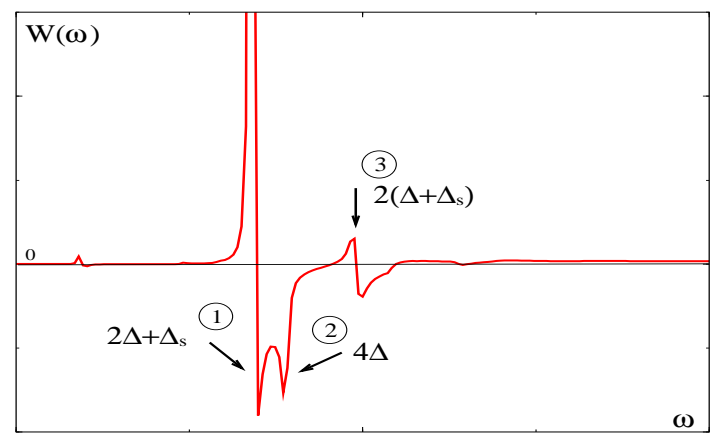

FIG. 26. The calculated frequency dependence of $W(\omega)=\frac{d^{2}}{d^{2} \omega}[\omega \operatorname{Re}[1 / \sigma(\omega)]]$ at $T \rightarrow 0$. This quantity is a sensitive measure of the fine structure in the optical response. The locations of the extrema are: (1) $2 \Delta+\Delta_{s}$, (2) $4 \Delta$, (3) $2 \Delta+2 \Delta_{s}$. Observe that the maximum shifts to a lower temperature, but the minimum remains at $2 \Delta+\Delta_{s}$. (From Ref. 109.)

For superconductors with pairing due to electronphonon interaction the fine structure of the optical conductivity has been analyzed by studying the second derivative of conductivity via $W(\omega)=\frac{d^{2}}{d^{2} \omega}\left(\omega \operatorname{Re} \sigma^{-1}(\omega)\right)$ which is proportional to $\alpha^{2}(\omega) F(\omega)$ where $\alpha(\omega)$ is an effective-ectron-phonon coupling, and $F(\omega)$ is a phonon DOS119. In Fig.26 we present the result of the strong coupling calculations of $W(\omega) 109$. There is a sharp maximum in $W(\omega)$ near $2 \Delta+\Delta_{s}$, which is followed by a deep minimum. This form is consistent with our analytical observation that for a flat gap (which we used in our numerical analysis), the first derivative of conductivity diverges at $\omega=2 \Delta+\Delta_{s}$. At a finite $T$ (a necessary attribute of a numerical solution), the singularity is smoothed, and the divergence is transformed into a maximum. Accordingly, the second derivative of the conductivity should have a 
maximum and a minimum near $2 \Delta+\Delta_{s}$. The numerical analysis shows that the maximum shifts to lower frequencies with increasing $T$, but the minimum moves very little from $2 \Delta+\Delta_{s}$, and is therefore a good measure of a magnetic "fingerprint".

Second, we note from Fig.26 that in addition to the maximum and the minimum near $2 \Delta+\Delta_{s}, W(\omega)$ has extra extrema at $4 \Delta$ and $2 \Omega_{\mathrm{t}}=2 \Delta+2 \Delta_{s}$. These are precisely the extra features that we expect: they are a primary effect due to a singularity in $\tilde{\Sigma}(\omega)$ at $\omega=3 \Delta$ and a secondary effect due to a singularity in $\tilde{\Sigma}(\omega)$ at $\omega=\Omega_{\mathrm{t}}$. The experimental discovery of these features will be a further argument in favor of spin-mediated pairing and the applicability of the spin-fermion model.

\section{COMPARISON WITH THE EXPERIMENTS ON CUPRATES}

In this section we compare the theoretical results for the spin-fermion model of the nearly antiferromagnetic Fermi liquid with the experimental data for optimally doped members of the $\mathrm{Bi}_{2} \mathrm{Sr}_{2} \mathrm{CaCu}_{2} \mathrm{O}_{8}$ and $\mathrm{YBa}_{2} \mathrm{Cu}_{3} \mathrm{O}_{7-y}$ families of cuprate superconductors. We make the assumption that at this doping level, absent the influence of a superstructure and imperfections that make the NMR lines in $\mathrm{Bi}_{2} \mathrm{Sr}_{2} \mathrm{CaCu}_{2} \mathrm{O}_{8}$ broad and difficult to interpret, the normal state behavior of $\mathrm{Bi}_{2} \mathrm{Sr}_{2} \mathrm{CaCu}_{2} \mathrm{O}_{8}$ will resemble closely that of $\mathrm{YBa}_{2} \mathrm{Cu}_{3} \mathrm{O}_{7-y}$. This enables us to take the two input parameters of the model from fits to NMR in the latter material. We then can compare theory and experiment in the normal state and as $T \rightarrow 0$ in the superconducting state. Finally, we discuss the general phase diagram of the cuprates and the pseudogap physics of these materials.

\section{A. Parameters of the model}

The two input parameters of the theory are the coupling constant $\lambda$ and the overall energy scale $\bar{\omega}=4 \lambda^{2} \omega_{\mathrm{sf}}$. Alternatively, we can re-express $\lambda$ as $\lambda=3 v_{F} \xi^{-1} /\left(16 \omega_{\mathrm{sf}}\right)$ and use $v_{F} \xi^{-1}$ and $\omega_{\text {sf }}$ as inputs. The values of $\omega_{\text {sf }}$ and $\xi$ can be extracted from the NMR measurements of the longitudinal and transverse spin-lattice relaxation rates, and from neutron scattering data, which measure $S(\mathbf{q}, \omega) \propto \omega /\left(\left(1+(\mathbf{q}-\mathbf{Q})^{2} \xi^{2}\right)^{2}+\left(\omega / \omega_{\text {sf }}\right)^{2}\right)$. We will primarily rely on NMR data for near optimally doped $\mathrm{YBa}_{2} \mathrm{Cu}_{3} \mathrm{O}_{6+\delta}$. The NMR analysis 135 , 194 yields a moderately temperature dependent $\omega_{\mathrm{sf}}$ and $\xi$ which take the values $\omega_{\text {sf }} \sim 15-20 \mathrm{meV}$ and $\xi \sim 2 a$ in the vicinity of $T_{\mathrm{cr}}$, which for slightly overdoped materials will be close to $T_{c}$. The neutron data from inelastic scattering (INS) experiments on the normal state are more difficult to analyze because of the background which increases the measured width of the neutron peak and because of the possible influence of weak intrinsic inhompgeneities on a global probe such as INS. The data show 175 that the dynamical structure factor in the normal state is indeed peaked at $\mathbf{q}=\mathbf{Q}=(\pi / a, \pi / a)$, and that the width of the peak increases with frequency and at $\omega=50 \mathrm{meV}$ reaches 1.5 of its value at $\omega=0$. A straightforward fit to the theory yields $\omega_{\mathrm{sf}} \sim 35-40 \mathrm{meV}$ and a weakly temperature dependent $\xi \sim a$ which are, as expected, larger than the $\omega_{\text {sf }}$ and smaller than the $\xi$ values extracted from NMR. We will be using $\omega_{\text {sf }} \sim 20 \mathrm{meV}$ and $\xi=2 a$ for further estimates.

The value of the Fermi velocity can be obtained from the photoemission data on $\mathrm{Bi}_{2} \mathrm{Sr}_{2} \mathrm{CaCu}_{2} \mathrm{O}_{8}$ at high frequencies, where the self-energy corrections to the fermionic dispersion become relatively minor. We note that because of problems related to the surface reconstruction in $\mathrm{YBa}_{2} \mathrm{Cu}_{3} \mathrm{O}_{6+\delta}$ the vast majority of high quality angular resolved photoemission spectroscopy (ARPES) experiments are performed on $\mathrm{Bi}_{2} \mathrm{Sr}_{2} \mathrm{CaCu}_{2} \mathrm{O}_{8}$, the material where there are much less reliable NMR experiments in part because of superstructure induced line broadening. The three groups that report MDC (momentum distribution curve) data for $\mathrm{Bi}_{2} \mathrm{Sr}_{2} \mathrm{CaCu}_{2} \mathrm{O}_{8}$ and momenta along the zone diagonal153 196.193 all agree that the value of the bare Fermi velocity along the diagonal (determined at higher energies where mass renormalization is assumed to be small) is rather high: $2.5-3 \mathrm{eV} \AA$, or $0.7-0.8 \mathrm{eVa}$ where $a \simeq 3.8 \AA$ is the $\mathrm{Cu}-\mathrm{Cu}$ distance. We can use the $t-t^{\prime}$ tight binding model for the electronic dispersion to relate this velocity with that at hot spots. Using the experimental facts that the Fermi surface is located at $\mathbf{k} \approx(0.4 \pi / a, 0.4 \pi / a)$ for momenta along the zone diagonal and at $\mathbf{k} \approx(\pi / a, 0.2 \pi / a)$ for $k_{x}$ along the zone boundary, we find $t \sim 0.2-0.25 \mathrm{eV}, t^{\prime} \approx-0.35 t$ and $\mu \approx-1.1 t$. These numbers agree with those used in numerical studies 197. The hot spots are located at $\mathbf{k}_{\mathrm{hs}}=(0.16 \pi, 0.84 \pi)$ and symmetry related points, and the velocity at a hot spot is then approximately half of that along zone diagonal. This yields $v_{F} \approx 0.35-0.4 \mathrm{eVa}$.

Combining the results for $v_{F}, \xi$ and $\omega_{\text {sf }}$, we obtain $\lambda \sim 1.5-2$. This in turn yields $\bar{\omega} \sim 0.2-0.3 \mathrm{eV}$. As an independent check of the internal consistency of these estimates, we compare theoretical and experimental values of the resonance spin frequency $\Delta_{s}$. As we said at the end of Section. $4, \Delta_{s} \simeq 0.2 \bar{\omega}$ for $\lambda=2$. Substituting the value of $\bar{\omega}$, we obtain $\Delta_{s}$ close to the experimental value of $40 \mathrm{meV}$. A smaller $\omega_{\mathrm{sf}}=15 \mathrm{meV}$ would require a slightly larger $\lambda$, but variations of this magnitude are certainly beyond the quantitative accuracy of our theory. 

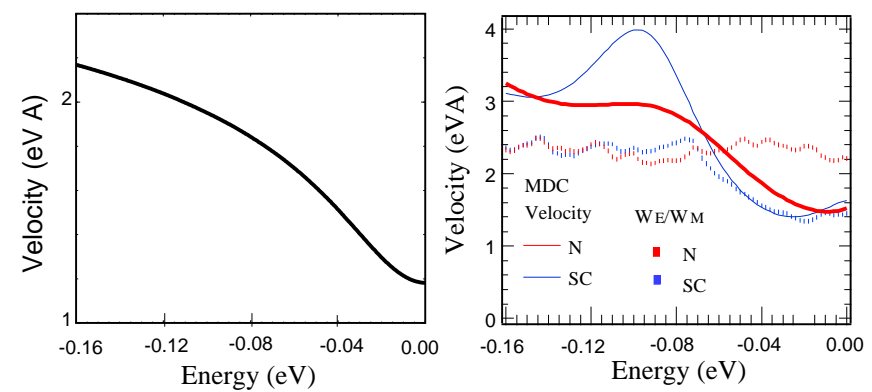

FIG. 27. a. The theoretical result for the effective velocity of the quasiparticles $v_{F}^{*}=v_{F} /\left(1+\partial \Sigma^{\prime}(\omega) / \partial \omega\right)$. For definiteness we used $\omega_{s f}=20 \mathrm{meV}, \lambda=1.7$ and bare velocity $v_{F}=3 \mathrm{eVA}$ along the diagonal. b. Experimental result for the effective velocity, extracted from the MDC dispersion 153 along the zone diagonal. Observe the bump in the frequency dependence of the velocity at $70-80 \mathrm{meV}$ in the data and at about $3-4 \omega_{s f}$ in the theory.

Away from hot spots, the effective coupling decreases as $\lambda(\mathbf{k})=\lambda /\left(1+(\delta k \xi)^{2}\right)^{1 / 2}$ where $\delta k$ is the momentum deviation from a hot spot along the Fermi surface. The largest $\delta k \xi$ is for $\mathbf{k}$ vectors along the zone diagonals. At optimal doping, ARPES data yield $\delta k^{\max } \sim 0.2 \pi / a \approx$ $0.6 / a 152$. We see that $\lambda$ is reduced by at most 1.7 as one moves from hot spots to the zone diagonal. A prediction of the model is that $\omega_{\mathrm{sf}}(k)$ increases at deviations from hot spots. This increase, however, should be at least partly compensated by the fact that $\omega_{\mathrm{sf}} \propto \sin \phi_{0}$, where $\phi_{0}$ is the angle between Fermi velocities at $\mathbf{k}$ and $\mathbf{k}+\mathbf{Q}$, with $\phi_{0} \simeq \frac{\pi}{2}$ in the vicinity of hot spots. $\phi_{0}$ tends to $\pi$ as $\mathbf{k}$ approaches the zone diagonal, and this reduces $\omega_{\mathrm{sf}}$. In view of this competing effect which we cannot fully control, we believe that the effective $\omega_{\text {sf }}(\mathbf{k})$ can best be obtained from the fit to the photoemission data, particularly from the MDC measurements of the electronic dispersion $\omega+\Sigma^{\prime}(\omega)=\epsilon_{\mathbf{k}}$. In Fig 27 we compare our $\left(1+\partial \Sigma^{\prime}(\omega) / \partial \omega\right)$ with the measured variation of the effective velocity $(\omega)$ of the electronic dispersion along zone diagonal 153 . We see that the theoretical dispersion has a bump at $\omega \sim 3 \omega_{\text {sf }}\left(\mathbf{k}_{\text {diag }}\right)$. The experimental curves lok quite similar and show a bump at $\sim 70-80 \mathrm{meV} 53.196193$. This yields $\omega_{\mathrm{sf}}\left(\mathbf{k}_{\text {diag }}\right) \sim 25 \mathrm{meV}$, a value only slightly larger than that near hot spots.

Note in passing that although $\delta k \xi$ does not vary much when $k$ moves along the Fermi surface, the fact that the Fermi velocity is fairly large implies that along the zone diagonal, $\epsilon_{\mathbf{k}_{\mathrm{F}}+\mathbf{Q}}$ is roughly $\sqrt{2} v_{\mathrm{F}} 0.2 \pi / a \approx 0.8 \mathrm{eV}$, i.e., it is comparable to the bandwidth. This implies that the Fermi-surface is very different from the nearperfect square that one would obtain for only nearest neighbor hopping. Furthermore, the fact that the Fermi velocity is large implies the physics at energies up to few hundred $\mathrm{meV}$ is confined to the near vicinity of the Fermi surface, when one can safely expand $\epsilon_{\mathbf{k}}$ to linear order in $\mathbf{k}-\mathbf{k}_{\mathrm{F}}$. Finally, van-Hove singularities (which we neglected) do play some role200201 but as $\epsilon_{(0, \pi / a)} \approx 0.34 t \sim 85 \mathrm{meV} \gg \omega_{\text {sf }}$, we expect that the van-
Hove singularity softens due to fermionic incoherence and should not substantially affect the physics. The value of $\epsilon_{(0, \pi / a)}$ might however be affected by an additional bilayer splitting which moves one of the bands closer to $\mathbf{k}=(0, \pi / a)$.

Finally, in the analysis of the spin-fermion model we have neglected the temperature dependence of the correlation length, and thus of $\omega_{\mathrm{sf}}$. Fits to NMR experiments on the near optimally doped member of the $\mathrm{YBa}_{2} \mathrm{Cu}_{3} \mathrm{O}_{6+\delta}$ family show that at $T_{\mathrm{cr}} \simeq T_{\mathrm{c}}$ both $\omega_{\mathrm{sf}}$ and $\xi$ display mean field behavior with $\xi^{-2} \simeq \xi_{0}^{-2}\left(1+T / T_{0}\right)$ and $\omega_{\mathrm{sf}} \xi^{2} \simeq 70 \mathrm{meV}$ almost independent on $T$.

From a theoretical perspective, the leading temperature dependence of $\xi$ arises from an interaction between spin-fluctuations and near the critical point in two dimensions has the form $\xi^{-2}(T)=\xi^{-2}(T=0)+B \Gamma_{4} T \log T$ where $B=O(1)$, and $\Gamma_{4}$ is the effective four boson interaction that is made out of fermions $7 \mathbf{7 8}, 171$. The $T \log T$ factor is the universal contribution from the bosonic loop, confined to momenta near $\mathbf{Q}$. The four-boson interaction has two contributions: one comes from low-energy fermions and is universal; the other comes from highenergy fermions and-depends on the fermionic bandwidth $W$. One can show 171 that the temperature correction to $\xi$ involves only the high-energy part of the interaction (this is what we labeled as $\Gamma_{4}$ ). The magnitude of $\Gamma_{4}$ can be easily estimated to be $\bar{g}^{2} / W^{3}$. Accordingly, the temperature correction to $\xi$ scales as $\frac{T}{\bar{g}}(\bar{g} / W)^{3}$. As we have remarked, the theory is universal as long as $\bar{g} \ll W$. In this limit, the temperature dependence of $\xi$ is obviously small and can be neglected. This is what we will do. Notice however that in the opposite limit, when $\bar{g} \gg W$, the full four-boson interaction differs from the lowest order term in $\bar{g}$ and might be estimated within an RPA-type summation. Estimates show that in this limit, the full $\Gamma_{4}$ is fully determined within the low-energy sector and scales as $O(1 / J)$ where $J \sim W^{2} / \bar{g}$ is the magnetic exchange integral. This in turn yields a much stronger temperature dependence of $\xi$ : $\xi^{-2}(T)-\xi^{-2}(T=0) \sim(T / J) \log T$. This result is similar to that obtained using a nonjinear $\sigma$ - model approach to near antiferromagnetisme2 204. The agreement becomes obvious in the limit of a large spin-fermion interaction (which, we recall, is the Hubbard $U$ if we derive the spinfermion model within the RPA); double occupancy is energetically unfavorable and the spin susceptibility obeys the constraint $\int d^{2} q d \omega \chi(\underline{\mathrm{q}}, i \omega) \propto 1-x$. This is equivalent to imposing a constraint on the length of the spin field in the nonlinear $\sigma$-model202 204 .

\section{B. The normal state}

In this section we compare the experimental and theoretical forms of the fermionic spectral function and optical conductivity in the normal state. No free parameters remain, since those which are needed to specify the model 
completely have been taken from NMR and ARPES experiments. The discussion will follow Ref. 51.

\section{The spectral function:}

The quasiparticle spectral function at various momenta is measured in angle resolved photoemission experiments. In a sudden approximation (an electron, hit by light, leaves the crystal without further interactions with other electrons and without paying attention to selection rules for the optical transition to its final state), the photoemission intensity is given by $I_{\mathbf{k}}(\omega)=A_{\mathbf{k}}(\omega) n_{F}(\omega)$ where $n_{F}$ is the Fermi function and $A_{\mathbf{k}}(\Omega)=(1 / \pi)|\operatorname{ImG}(\mathbf{k}, \Omega)|$ is the spectral function.
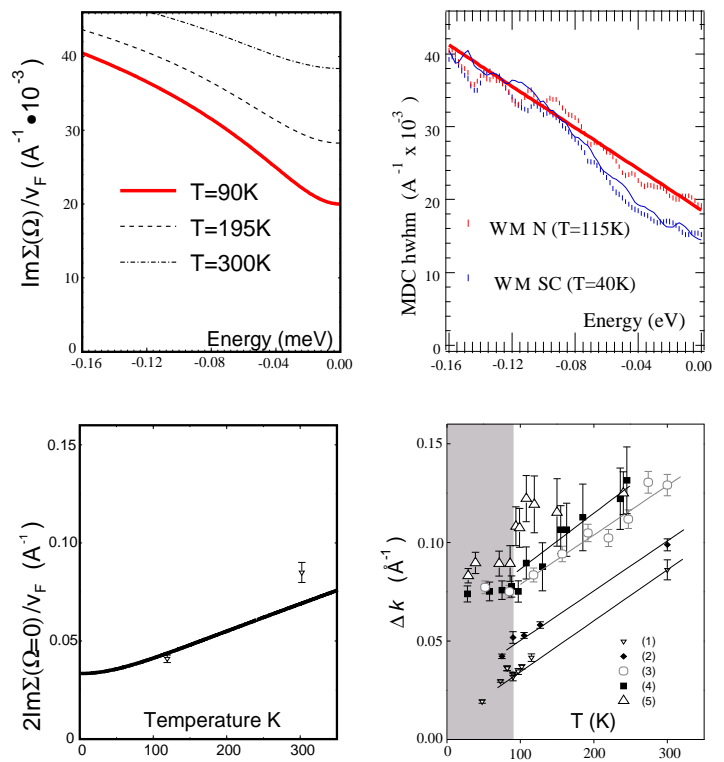

FIG. 28. A comparison between the theoretical results of the spin fermion model and the photoemission MDC data. For the Lorentzian line-shape of the MDC signal, observed in experiments, the MDC hwhm equals to $\Sigma^{\prime \prime} / v_{F}$. Upper panel - the results for the MDC hwhm vs frequency at a given $T$. The experimental results are taken from 153. Lower panel the MDC fwhm vs temperature at $\Omega=0$. The experimental results (right figure and the points on-the left figure) are taken from 196. The figure is taken from 11 .

We first use our form of the fermionic self-energy to fit MDC data which measure the width of the photoemission peak as a function of $\mathbf{k}$ at a given frequency. In Fig. 28 we compare the theoretical results for $\Delta k=\Sigma^{\prime \prime}(\mathbf{k}, \Omega) / w_{T_{3}}$ with the measured $\Delta k$ versus frequency at $T \sim 100 \mathrm{~K} 153$ and versus temperature at $\Omega \rightarrow 0$ 196. We used $\lambda=1.7$ and $\omega_{\mathrm{sf}}=20 \mathrm{meV}$. The slope of $\Delta k$ is chiefly controlled by $\lambda$. We obtain rather good agreement with the data, both for the frequency and temperature dependence of the self-energy. On the other hand, the magnitude of our $\Sigma^{\prime \prime}$ is smaller than that found in the experimental data. To account for the values of $\Delta k$, we had to add a constant of about $70 \mathrm{meV}$ to $\Sigma^{\prime \prime}$. The origin of this constant is unclear and explaining it is presently a challenge to the theory _ t may be the effect of elastic scattering by impurities 205, but the large value of this constant implies that it is more likely the contribution from scattering channels that we ignored. It is essential, however, that the functional dependence of $\Sigma^{\prime \prime}(\Omega, T)$ can be captured in the spin-fluctuation approach.

In Fig 29 we present the results for the half width at half maximum of the EDC (energy distribution curve) which measures fermionic $I_{\mathbf{k}}(\Omega)=A_{\mathbf{k}}(\Omega) n_{F}(\Omega)$ as a function of frequency at a given $\mathbf{k}$. For a Lorentzian lineshape, the EDC hwhm is given by $\Sigma^{\prime \prime}(\Omega) /\left(1+\Sigma^{\prime}(\Omega) / \Omega\right)$. The data are taken at $T=115 \mathrm{~K} 153$. We see that the theoretical slope agrees reasonably well with the experimental one. The visible discrepancy is most likely associated with the fact that the experimental line-shape is not a perfect Lorentzian.
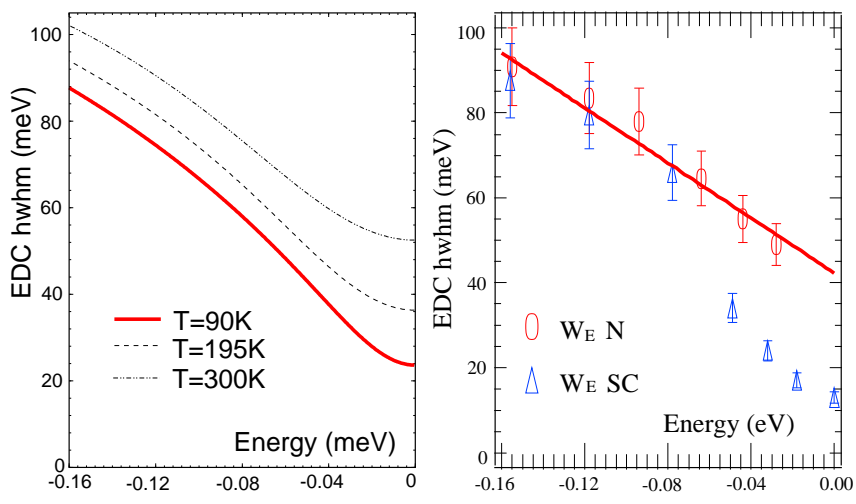

FIG. 29. A comparison of the theoretical result for $\Sigma^{\prime \prime}(\Omega) /\left(1+\Sigma^{\prime}(\Omega) / \Omega\right)$ with the experimental hwhm of the EDC dispersion from 153 . The figure is taken from 51 .

\section{The optical conductivity}

In Fig.30 we compare the theoretical results for the conductivity 109 with the experimental data for $\sigma_{1}$ and $\sigma_{2}$ at different temperatures 190 . The theoretical results are obtained using the same $\lambda=1.7$ and $\omega_{\text {sf }}=20 \mathrm{meV}$ as in the fit to the photoemission data along zone diagonals. Changing $\lambda$ affects the ratio $\sigma_{2} / \sigma_{1}$ at high frequencies, but does not change the functional forms of the conductivities 109 . The value of the plasma frequency was adjusted to match the d.c. conductivity and $\Sigma^{\prime \prime}$ extracted from the MDC photoemission data using $v_{F} \sim 3 \mathrm{eVA}$. This adjustment yields $\omega_{\mathrm{pl}} \sim 20000 \mathrm{~cm}^{-1}$. This value is somewhat larger than $\omega_{\mathrm{pl}} \sim 16000 \mathrm{~cm}^{-1}$ obtained perimentally by integrating $\sigma_{1}$ up to about $2-2.5 \mathrm{eV} 158,190191$, however it agrees with the theoretical result 109 that the sum rule for $\sigma_{1}(\omega)$ is exhausted only at extremely high frequencies of about $50 \bar{\omega}$, that are much larger than $2 \mathrm{eV}$. 


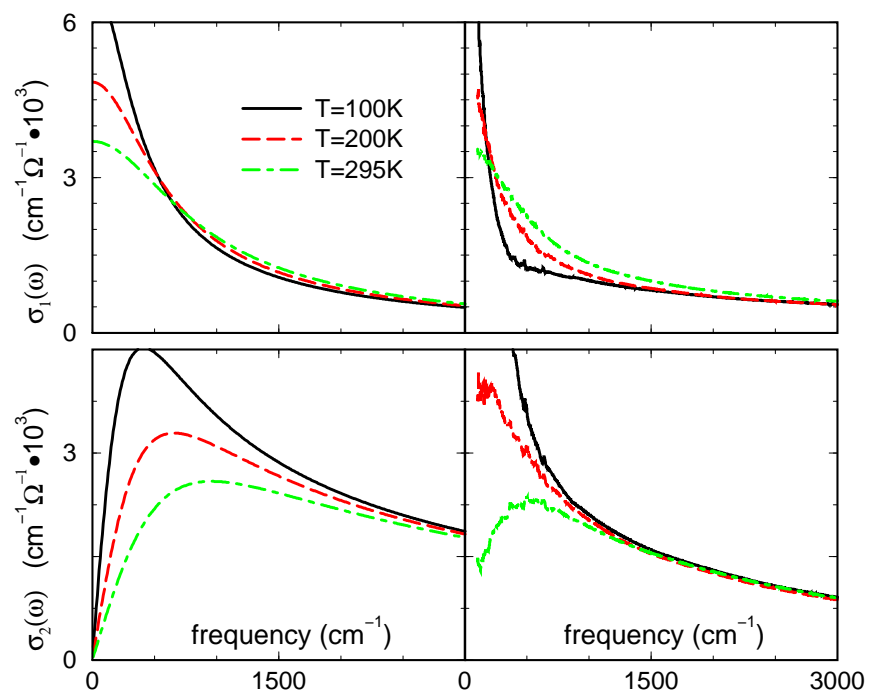

FIG. 30. The theoretical and experimental results for the real and imaginary parts of optical conductivity. The data are from 190. The figure is taken from 51 .

We see that theoretical calculations of $\sigma_{1}(\omega)$ and $\sigma_{2}(\omega)$ capture the essential features of the measured forms of the conductivities. In particular, the curves of $\sigma_{1}$ at different temperatures cross such that at the lowest frequencies, the conductivity decreases with $T$ while at larger frequencies it increases with $T$, a behavior seen in Ref. 195, 191. The imaginary part of conductivity decreases with $T$ at any frequency, and the peak in $\sigma_{2}(\omega)$ increases in magnitude and shifts to lower $T$ with decreasing $T$ 190.191]189. At frequencies above $1500 \mathrm{~cm}^{-1}$ both $\sigma_{1}$ and $\sigma_{2}$ depend weakly on $T$ and are comparable in amplitude.

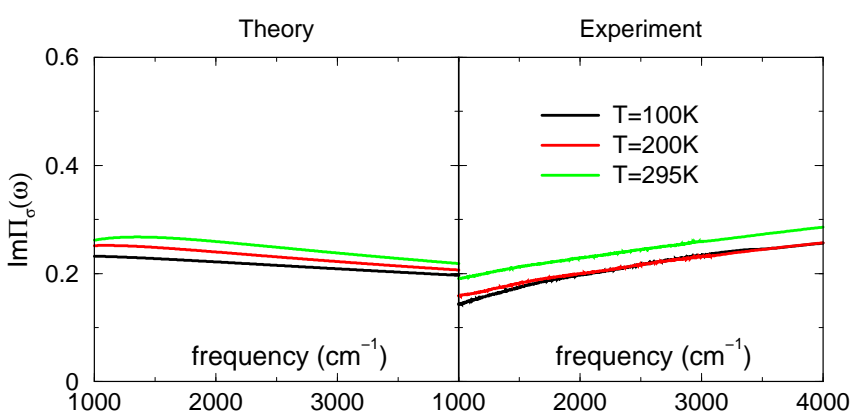

FIG. 31. The theoretical and experimental results for $\Pi_{\sigma}^{\prime \prime}(\omega)=4 \pi \sigma_{1} \omega / \omega_{p l}^{2}$ (from 51). The data are from 190 .

To make the comparison more quantitative, in Fig 31 we present experimental and theoretical results for the imaginary part of the full particle-hole polarization bubble $\Pi_{\sigma}^{\prime \prime}(\omega)=4 \pi \sigma_{1} \omega / \omega_{\mathrm{pl}}^{2}$. Theoretically, at $T=0, \Pi_{\sigma}^{\prime \prime}(\omega)$ saturates at a value of about 0.2 independently of $\lambda$ and remains almost independent of frequency over a very wide frequency range 109. The experimental data also clearly show a near saturation of $\Pi_{\sigma}^{\prime \prime}$ at a value close to 0.2 .

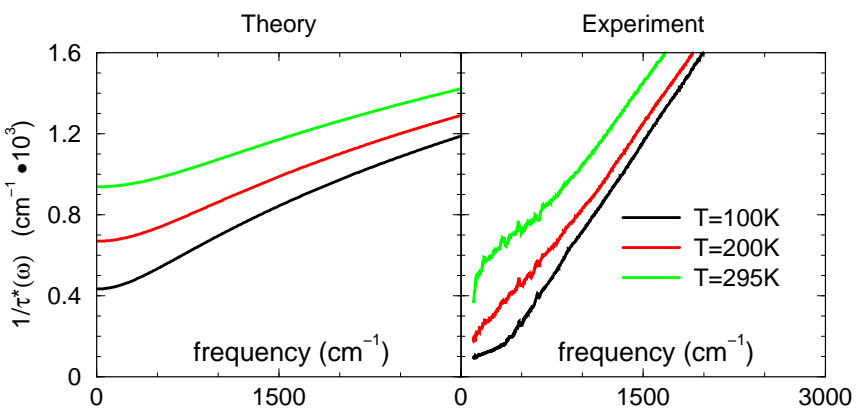

FIG. 32. The theoretical and experimental results for $1 / \tau^{*}=\omega \operatorname{Re} \sigma / \operatorname{Im} \sigma($ from 51$)$. The data are from 190 .

The agreement between theory and experiment is, however, not a perfect one. In Fig. 32 we show theoretical and experimental results for $1 / \tau^{*}=\omega \sigma_{1} / \sigma_{2}$. The advantage of comparing $1 / \tau^{*}$ is that this quantity does not depend on the unknown plasma frequency. We see that while both experimental and theoretical curves are linear in frequency, the slopes are off roughly by a factor of 3. This discrepancy is possibly related to the fact that in the spin-fermion model, $\Pi_{\sigma}^{\prime}(\omega)$ at high epongh frequencies is roughly 3 times larger than $\Pi_{\sigma}^{\prime \prime}(\omega) 109$, and hence $\sigma_{2} / \sigma_{1} \sim 3$, whereas experimentally $\sigma_{1}$ and $\sigma_{2}$ are comparable in magnitude at high frequencies. The discrepancy in $1 / \tau^{*}$ indicates that either the averaging over the Fermi surface, vertex corrections inside a particle-hole bubble, or RPA-type corrections to the conductivity 192 play some role. Still, Figs. 30 and 31 indicate that the general trends of the behavior of the conductivities near optimal doping are reasonably well captured within the spin-fluctuation approach.

\section{The superconducting state}

In this section, we apply our results from Section 5 to cuprates and examine to what extent the "fingerprints" of spin-fluctuation pairing have been detected in experiments on optimally doped high $T_{c}$ materials.

\section{The spin susceptibility}

The major prediction of the spin fermion model for the spin susceptibility in the superconducting state is the emergence of the resonance peak in $\chi^{\prime \prime}(\mathbf{Q}, \omega)$ at $\omega=\Delta_{s}$ if $\Delta_{s}<2 \Delta$. The magnitude $\Delta_{s}$ is fully determined within the theory and is chiefly set by the magnitude of the superconducting gap as well as the energy scale of magnetic fluctuations in the normal state, $\omega_{\mathrm{sf}}$. For small doping concentration $\Delta_{s} \propto \xi^{-1}$ must decrease as one approaches the antiferromagnetic state. The resonance mode is confined to a small region in momentum space (where it is of high intensity). For momenta away from $\mathbf{Q}$ and its close vicinity, magnetic excitations couple to gapless, nodal 
quasiparticles and become overdamped, eliminating the resonance mode.

In Fig. 33 we show representative experimental data for $\chi^{\prime \prime}(\mathbf{Q}, \omega)$ showing the resonance peak at $\omega \approx 41$ $\mathrm{meV}$ for optimally doped $\mathrm{YBa}_{2} \mathrm{Cu}_{3} \mathrm{O}_{6.9} 174$. As noted earlier, the position of the peak is consistent with the prediction of the spin fermion model. Similar behavior is found in $\mathrm{Bi}_{2} \mathrm{Sr}_{2} \mathrm{CaCu}_{2} \mathrm{O}_{8}{ }_{163}$; here the peak is at $43 \mathrm{meV}$. With underdoping, the measured resonance energy goes down 161 162. In strongly underdoped $\mathrm{YBa}_{2} \mathrm{Cu}_{3} \mathrm{O}_{6.6}$, it is approximately $25 \mathrm{meV}{ }^{161}$. The existence of the peak and the downturn with underdoping agree with the predictions of the spin-fluctuation theory. Further, the measured amplitude of $\chi^{\prime \prime}(\mathbf{Q}, \omega)$ decreases above the peak, but increases again for $60-80 \mathrm{meV162,174}$. This might indeed be a $2 \Delta$ effect, which appears naturally within the model.
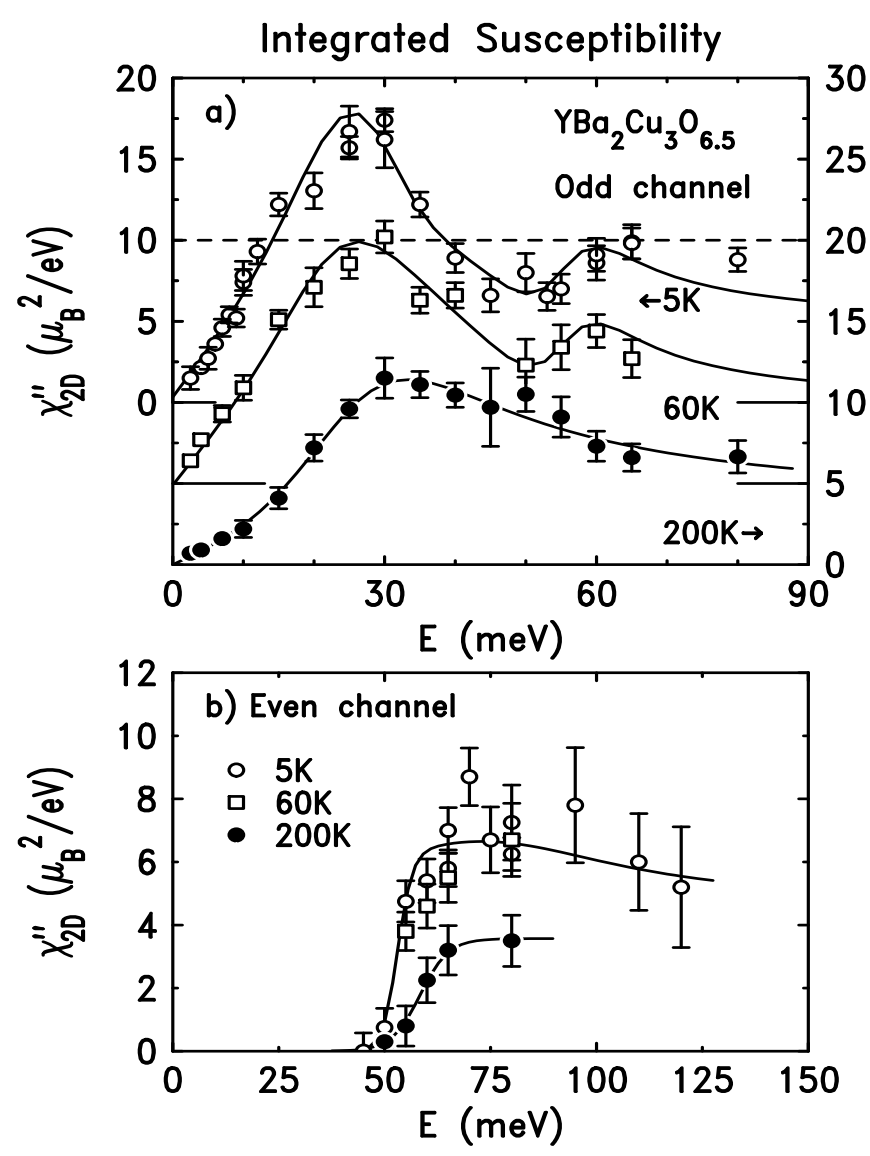

FIG. 33. Inelastic neutron scattering intensity for momentum $\mathbf{Q}=(\pi, \pi)$ as function of frequency for $\mathrm{YBa}_{2} \mathrm{Cu}_{3} \mathrm{O}_{6.5}$. Data from Ref. 161 .

The full analysis of the resonance peak requires more care as (i) the peak is only observed in two-layer materials, and only in the odd channel, (ii) the momentum dispersion of the peak is more complex than that for magnons 175 , (iii) the peak broadens with underdoping161.162, and (iv) in underdoped materials, the peak emerges at the onset of the pseudogap and only sharpens up at $T_{c} 162$ 174. All these features, already present on the level of a weak coupling approach 127120 have been explained within the spin-fermion mode131,102. The broadening of the peak was recently studied in detail in Ref.156. The explanation of these effects, however, requires careful analysis of the details of the electronic structure and is beyond the scope of this Chapter.

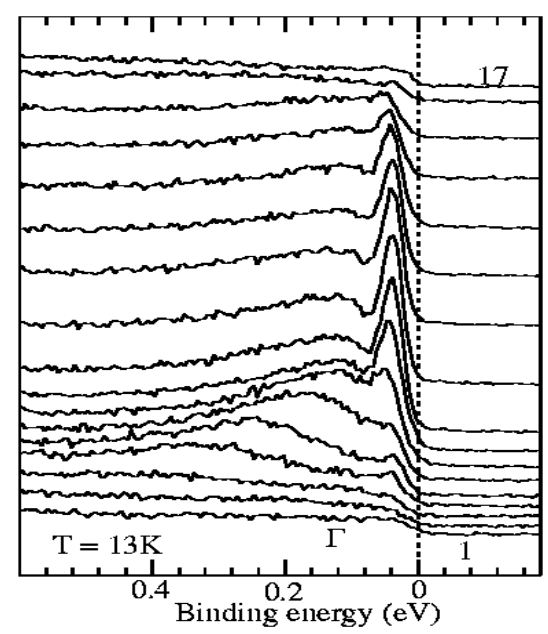

FIG. 34. ARPES spectrum for near optimally doped Bi2212 for momenta close to the hot spots. Data from Ref. 147 .

\section{The spectral function:}

The predictions of our approach are a peak-dip structure of the spectral function, with a weakly dispersing peak at $\omega \approx \Delta$ and a peak-dip distance $\approx \Delta_{s}$. On the other hand we expect a broad incoherent peak which disperses like $\epsilon_{\mathbf{k}}^{2} / \bar{\omega}$. In Fig. 34 we present ARPES data for near optimally doped Bi2212 with $T_{c}=87 \mathrm{~K}$ for momenta near a hot spot 147 . The intensity displays the predicted peak/dip/hump structure. A sharp peak is located at $\sim 40 \mathrm{meV}$, and the dis is at $80 \mathrm{meV}$ such that the peak-dip distance is $42 \mathrm{meV} 447$. In the spin-fluctuation theory, the peak-dip distance is the energy of the INS resonance peak frequency 127,170 . The neutron scattering data on Bi2212 with nearly the same $T_{c}=91 \mathrm{~K}$ yielde63 $\Delta_{s}=43 \mathrm{meV}$, in excellent agreement with this prediction. Furthermore, with underdoping, the peak-dip energy difference decreases and, within error bars, remains equal to $\Delta_{s}$. This behavior is illustrated in Fig. 35 . 
In Fig 36 we present experimental results for the variation of the peak and hump positions with the deviation from the Fermi surface. These show that the hump disperses with $\mathbf{k}-\mathbf{k}_{\mathrm{F}}$ and eventually recovers the position of the broad maximum in the normal state. At the same time, the peak shows little dispersion, and does not move further in energy than $\Delta+\Delta_{s}$. Instead, the amplitude of the peak dies off as $\mathbf{k}$ moves away from $\mathbf{k}_{\mathrm{F}}$. This behavior is again fully consistent with the theoretical predictions 17251.

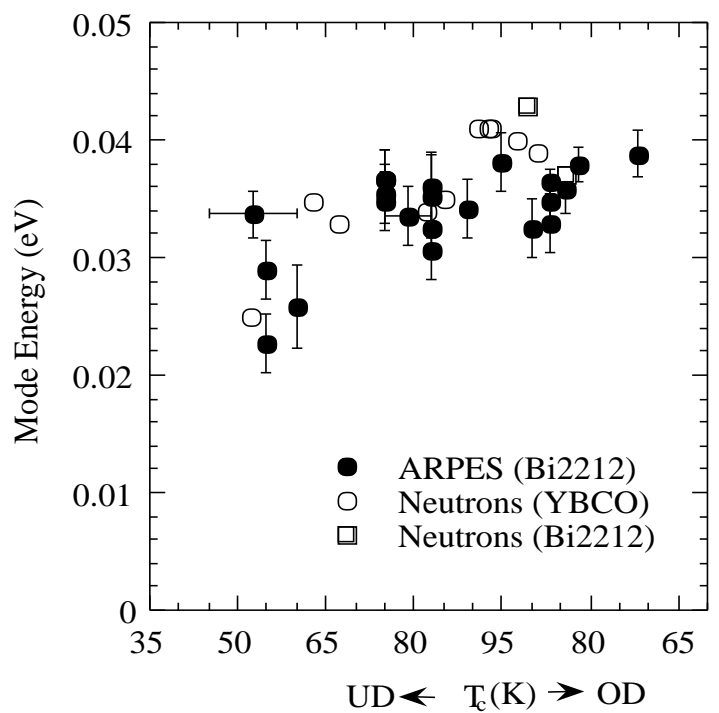

FIG. 35. The experimental peak-dip distance at various doping concentrations compared with $\Delta_{s}$ extracted from neutron measurements. Data from Ref.[30]. The theoretical result is presented in Fig. 19.

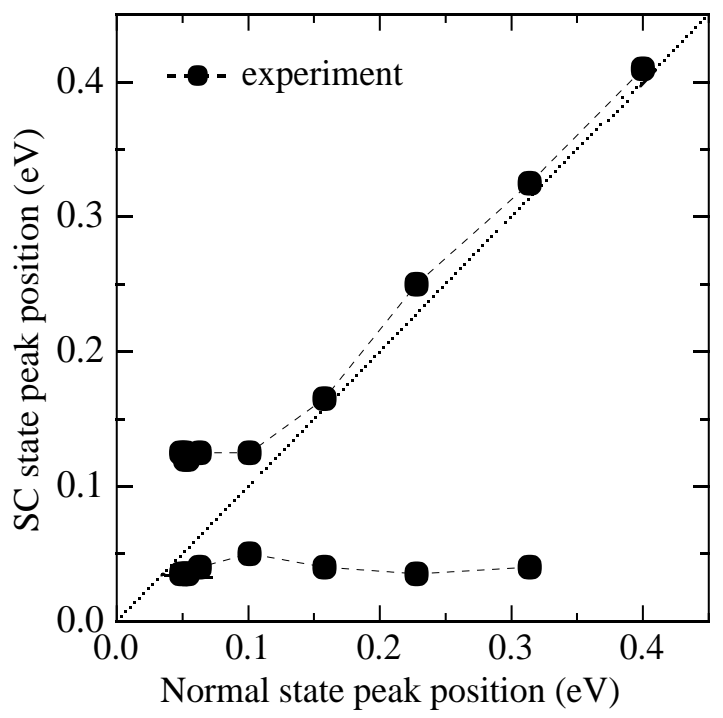

FIG. 36. The dependence of the experimental peak (flat curve) and hump (dispersing curve) positions on the deviation from the Fermi surface. The hump disperses with $\mathbf{k}-\mathbf{k}_{\mathrm{F}}$ (dotted line) and eventually recovers the position of the broad maximum in the normal state, while the peak position changes little with the deviation from $\mathbf{k}_{\mathrm{F}}$. Data from Ref. 147. The theoretical result is presented in Fig. 19.

We regard the presence of the dip at $\Delta+\Delta_{s}$, and the absence of the dispersion of the quasiparticle peak as two major "fingerprints" of strong spin-fluctuation scattering in the spectral density of cuprate superconductors.

\section{The density of states:}

The fermionic DOS $N(\omega)$ is proportional to the dynamical conductance $d I / d V$ through a superconductorinsulator-normal metal (SIN) measured at $\omega=\mathrm{eV}$ where $V$ is the applied voltage 124 . The key prediction of our approach is the occurrence of a dip in the DOS at an energy $\approx \Delta_{s}$ away from the peak at $\omega=\Delta$. The drop in the DOS at $\Omega_{\mathrm{t}}=\Delta+\Delta_{s}$ from Eq. 89 can be understood in terms of SIN conductance as follows: when the applied voltage, $V$, equals $\Omega_{\mathrm{t}} / e$ an electron that tunnels from a normal metal can emit a spin excitation and fall to the bottom of the band, losing its group velocity. This loss leads to a sharp reduction of the current and produces a drop in $d I / d V$. This process is shown schematically in Fig23.

SIN tunneling experiments have been performed on YBCO and Bi2212 materials 154. Similar results have been recently obtained by Davis et al.150. At low and moderate frequencies, the SIN conductance displays a behavior which is generally expected in a $d$-wave superconductor, i.e., it is linear in voltage for small voltages, and has a peak at $e V-$ where $\Delta$ is the maximum value of the $d$-wave gap 154,150 The value of $\Delta$ extracted from tunneling agrees well with the maximum valye of the gap extracted from ARPES measurement_151, 153. At frequencies larger than $\Delta$, the measured SIN conductance displays an extra dip-hump feature which become visible at around optimal doping, and grows in amplitude with underdoping 154. At optimal doping, the distance between the peak at $\Delta$ and the dip is around $40 \mathrm{meV}$. This is consistent with $\Delta_{s}$ extracted from neutron measurements.

\section{SIS tunneling:}

The major prediction of the spin-fermion model for the SIS tunneling conductance, $S(\omega)$, is the emergence of a singularity at $\omega=2 \Delta+\Delta_{s}$. As mentioned above, this singularity is likely softened due to thermal excitations or non-magnetic scattering processes and transforms into a dip slightly below $2 \Delta+\Delta_{s}$, and a hump at a frequency larger than $2 \Delta+\Delta_{s}$. 
data as an additional strong argument in favor of the magnetic scenario for superconductivity.

\section{Optical and Raman response:}

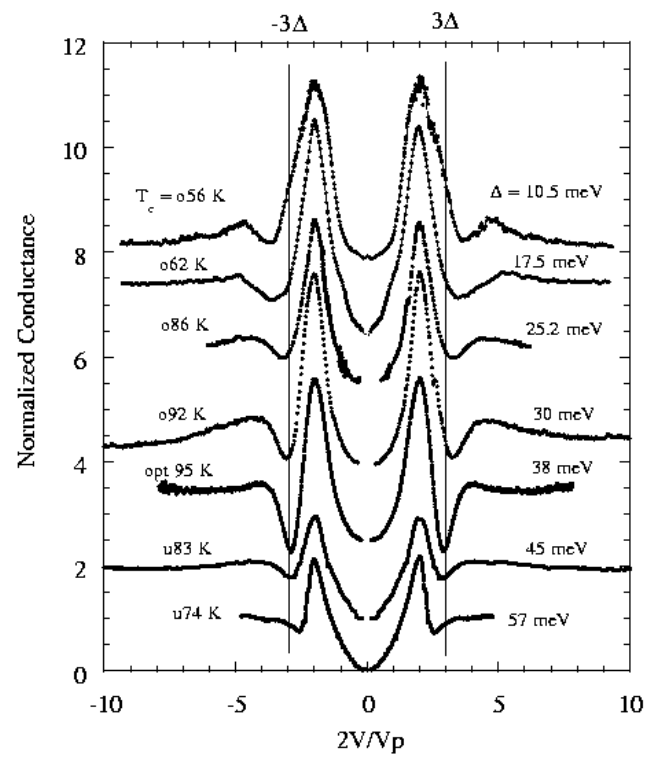

FIG. 37. SIS tunneling conductance normalized by $\Delta$ for Bi2212 materials ranging from overdoped (top curves) to underdoped (bottom curves) from Ref. 157. The peak dip distance increases for increasing doping and saturates at around $3 \Delta$ as expected in our theory. The corresponding theoretical result is presented in Fig. 22.

Recently, Zasadzinski et al. obtained both new data and carefully examined their previous SIS tunneling data for a set of Bi2212 materials ranging from overdoped to underdoped 157. Their data, presented in Fig. 37 show that in addition to the peak at $2 \Delta$, the SIS conductance displays the dip and the hump at larger frequencies. The distance between the peak and the dip (which approximately equals $\Delta_{s}$ in the spin fluctuation model179.149) is close to $2 \Delta$ in overdoped Bi2212 materials, but goes down with underdoping. Near optimal doping, this distance is around $40 \mathrm{meV}$. For an underdoped, $T_{c}=74 \mathrm{~K}$, material, the peak-dip distance is reduced to about $30 \mathrm{meV}$. These results are in qualitative and quantitative agreement with ARPES and neutron scattering data, as well as with the theoretical estimates. The most important aspect is that with underdoping, the experimentally measured peak-dip distance progressively shifts down from $2 \Delta$. This downturn deviation from $2 \Delta$ is a key feature of the spin-fluctuation mechanism. We regard the experimental verification of this feature in the SIS tunneling
Theoretical considerations show that optical measurements are much better suited than Raman measurements to search for the "fingerprints" of a magnetic scenarid49. For the optical conductivity we predict a singular behavior at energies $2 \Delta+\Delta_{s}, 4 \Delta, 2 \Delta+2 \Delta_{s}$, which can be amplified if one considers the second derivative of conductivity via $W(\omega)=\frac{d^{2}}{d^{2} \omega}\left(\omega \operatorname{Re} \sigma^{-1}(\omega)\right)$. Evidence for strong coupling effects in the optical conductivity in superconducting cuprates has been reported in Refs. 158,160, 183, 184.

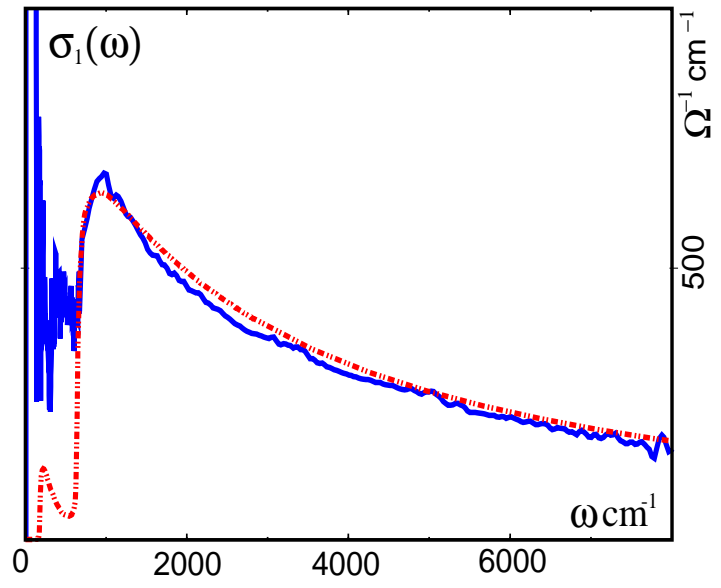

FIG. 38. A comparison between theoretical and experimental results for the optical conductivity (from Ref. 109). The experimental data are from Ref 158 .

We first discuss the form of $\sigma_{1}(\omega)$. In Fig. 38 we compare the theoretical result for $\sigma_{1}(\omega) 109.149$ with the experimental data by Puchkov et al 158 for optimally doped $\mathrm{YBa}_{2} \mathrm{Cu}_{3} \mathrm{O}_{6+\delta}$ in the superconducting state. The parameters are the same as in the normal state fits. As the theoretical formula does not include the contributions from the nodes, the comparison is meaningful only for $\omega>2 \Delta$. We see that the frequency dependence of the conductivity at high frequencies agrees well with the data. The measured conductivity drops at about $100 \mathrm{meV}$ in rough agreement with $2 \Delta+\Delta_{s}$ which for $\Delta \approx 30 \mathrm{meV}$ and $\Delta_{s} \approx 40 \mathrm{meV}$ is also around $100 \mathrm{meV}$. The good agreement between theory and experiment is also supportive of our argument that the momentum dependence of the fermionic dynamics becomes irrelevant at high frequencies, and fermions from all over the Fermi surface behave as if they were at hot spots.

We next consider the singularities in the frequency dependence of the conductivity in more detail and compare the theoretical and experimental results for $W(\omega)=$ $\frac{d^{2}}{d^{2} \omega}\left(\omega \operatorname{Re} \sigma^{-1}(\omega)\right)$. The theoretical result for $W(\omega)$ is pre- 
sented in Fig.26 The experimental result for $W(\omega)$ in YBCO is shown in Fig.39. We see that the theoretical and experimental plots of $W(\omega)$ look rather similar, and the relative intensities of the peaks are at least qualitatively consistent with the theory. We identify (see explanations below) $2 \Delta+\Delta_{s}$ with the deep minimum in $W(\omega)$. This identification, that is consistent with the analysis of $\sigma_{1}(\omega)$, yields $2 \Delta+\Delta_{s} \approx 100 \mathrm{meV}$. Identifying the extra extrema in the experimental $W(\omega)$ with $4 \Delta$ and $2 \Delta+2 \Delta_{s}$, respectively, we obtain $4 \Delta \sim 130 \mathrm{meV}$, and $2 \Delta+2 \Delta_{s} \sim 150 \mathrm{meV}$. We see that three sets of data are consistent with each other and yield $\Delta \sim 30 \mathrm{meV}$ and $\Delta_{s} \sim 40-45 \mathrm{meV}$. The value of $\Delta$ is in good agreement with tunneling measurements 186 , and $\Delta_{s}$ agrees well with the resonance frequency extracted from neutron measurements 161 . Indeed, the analysis of a second derivative of a measured quantity is a very subtle procedure. The good agreement between the theory and experiment is promising but has to be verified in further perimental studies. Still, theoretical calculations 109,49 clearly demonstrate the presence and observability of these "higher harmonics" of the optical response at $4 \Delta$ and $2 \Delta+2 \Delta_{s}$.

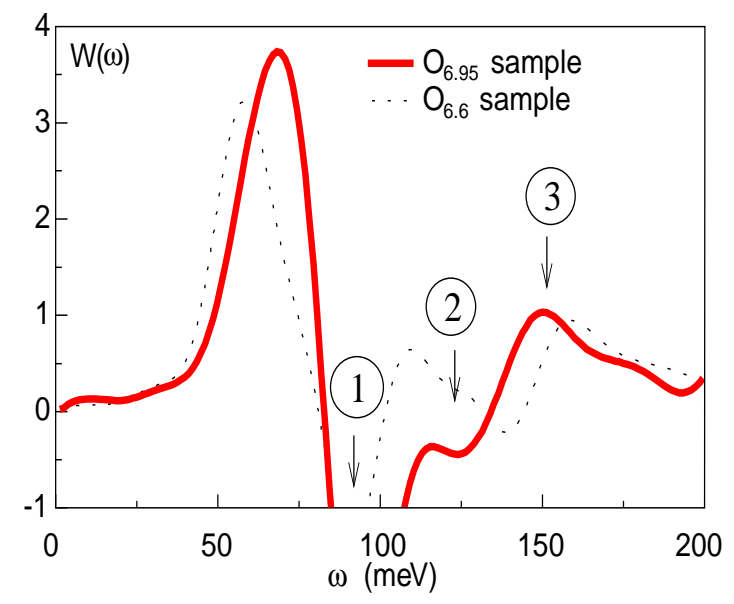

FIG.

39.

Experi-

mental results for $W(\omega)=\frac{d^{2}}{d^{2} \omega}\left(\omega \operatorname{Re} \sigma^{-1}(\omega)\right)$ from Ref. 160. The theoretical result is presented in Fig. 26. The position of the deep minimum agrees well with $2 \Delta+\Delta_{s}$. The extrema at higher frequencies are consistent with $4 \Delta$ and $2\left(\Delta+\Delta_{s}\right)$ predicted by the theory.

Finally, we comment on the position of the $2 \Delta+\Delta_{s}$ peak and compare the results of Abanov et al109.149 with those by Carbotte et al. .60 . Theoretically, at $T=0$ and in clean limit, the maximum and minimum in $W(\omega)$ are located at the same frequency. At a finite $T$, however they quickly move apart (see Fig 26). Carbotte et al. 160 focused on the maximum in $W(\omega)$ and argued that it is located at $\Delta+\Delta_{s}$ instead of $2 \Delta+\Delta_{s}$. We see from Fig 26 that the maximum in $W(\omega)$ shifts to a lower frequency with increasing temperature and over some $T$ range is located close to $\Delta+\Delta_{s}$. On the other hand, the minimum in $W(\omega)$ moves very little with increasing $T$ and virtually remains at the same frequency as at $T=0$. This result suggests that the minimum in $W(\omega)$ is a more reliable feature for comparisons with experiments. This conclusion is in agreement with recent conductivity data on optimally doped $\mathrm{Bi} 2212190$. $W(\omega)$ extracted from these data shows a strong downturn variation of the maximum in $W(\omega)$ with increasing temperature, but the minimum in $W(\omega)$ is located at around $110 \mathrm{meV}$ for all temperatures.

\section{Experimental facts that we cannot yet describe}

There are several experimental results that we do not understand. First are the results by Ando, Boebinger and collaborators 214216 on the behavior of the Lanthanum and Bismuthate based superconductors in magnetic fields sufficiently strong to (almost) destroy superconductivity. For doping levels close to the optimal one, they found that the resistivity at low temperatures continues to be linear in $T$ with the same slope seen at higher temperatures. If the assumption that the magnetic field destroys superconductivity but otherwise does not affect the system properties is correct, this result poses a problem for the spin-fluctuation model as the latter yields a linear in $T$ resistivity over a wide range of temperatures, but only for $T$ larger than a fraction of $\omega_{\mathrm{sf}}$. To account for these data one might have to invoke some kind of quantumcritical physics associated with the opening of the pseudogap (see below).

Another experiment that is not yet understood is the measurement of the Hall angle, $\theta_{H} \equiv \rho_{x y} / \rho_{x x}$ which shows an incredibly simple behavior, $\cot \theta_{H} \propto T^{2} 207$ and also displays a particular frequency behavior $200,210.211$. The orbital magnetoresistance $\Delta \rho / \rho$ also behaves in quite an unusual way, violating Kohler's rule, according to which $\Delta \rho / \rho$ is a function of $H^{2} / \rho^{2}$, independent of $T$, where $H$ is the applied magnetic field. Some of this physics is already captured in the semi-phenomenological calculations by Stojkovich and Pines212; however problems remain. In the description based on the spinfermion model the technical problem not yet solved is how to include in a controlled way vertex corrections which are not small; in one of the vertices for the Hall conductivity the momentum transfer is small. Some progress with these calculations have been recently made by Katami and collaborators217. Another explanation of the Hall data has recently been proposed by Abrahams and Varma208.

Yet another unanswered question, already noted above, is the origin of a large (almost $100 \mathrm{meV}$ ), frequency and temperature independent contribution to the selfenergy that one has to invoke in order to fit conductivity and ARPES data. It could, in principle, be due to inelastic scattering by impurities, but its very large value makes this explanation problematic.

Electronic Raman scattering reveals further puzzling 
behavior: in all geometries one observes a frequency independent behavior over a very large energy scale, frequently referred to as the positive background. Moreover, the overall size of the background is very different in different geometries 17 . 179.

There are also uncertainties associated with reconciling the incommensurability of the magnetic response in the normal state of 214 material 142 with the commensurate peaks required to obtain a consistent explanation of ${ }^{17} \mathrm{O}$ and ${ }^{63} \mathrm{Cu}$ NMR experiments, but these are not likely to pose fupdamental problems to the spin-fluctuation approach 135213.

Finally, the claim of universality of the low-energy behavior relies heavily on the existence of a quantum critical point at which the antiferromagnetic correlation length diverges. In real materials there are indications that the transition to antiferromagnetism is actually of first order. In this situation, the theory we described is valid only if there still exists a substantial region in parameter space where the system is critical before it changes its behavior discontinuously. NMR and neutron scattering experiments on optimally doped cuprates seem to support such behavior. Another reason for concern is the role of disorder and inhomogeneities. Despite enormous progress in sample fabrication, cuprates often tend to be very heterogeneous materials. It has been established in several cases that these aspects are actually intrinsic, forcing one to include effects due to inhomogeneities and disorder into the theoretical description 231.

\section{E. Phase diagram}

In this section we discuss in detail the experimental phase diagram of cuprate superconductors and comment on the origin of the pseudogap behavior found for small charge carrier concentrations.

From a general perspective, the key to understanding of cuprate superconductors is identifying the nature of the protected behavior of the novel states of matter encountered in the insulating, conducting, and superconducting states as one varies doping and temperature, including the possible existence of one or more quantum critical points. Consider first the $\mathrm{YBa}_{2} \mathrm{Cu}_{3} \mathrm{O}_{7-\delta}$ system on which the generic phase diagram of Fig.1 was based 135. A somewhat similar diagram based on transport measurements was independently proposed by Hwang et al. 36 , while one based on specific heat and susceptibility mesurements has been proposed recently by Tallon et al.138. As discussed in the Introduction, in addition to the $T_{c}$ line, there are two crossover or phase transition lines in Fig.1. The upper line $T=T_{\text {cr }}$ is defined experimentally by a maximum in the temperature dependent uniform magnetic susceptibility, $\chi_{0}$. It has been further characterized 135 as the temperature at which the antiferromagnetic correlation length $\xi$ is of the order $\mathrm{Cu}-\mathrm{Cu}$ lattice spacing (Barzykin and Pines used a criterion $\left.\xi\left(T_{\mathrm{cr}}\right)=2 a\right)$. The lower line $T=T^{*}$ may be defined experimentally as the temperature at which the product of the copper spin-lattice relaxation time, ${ }^{63} T_{1}$ and the temperature, $T$, reaches its minimum value. In the $\mathrm{Bi}_{2} \mathrm{Sr}_{2} \mathrm{CaCu}_{4} \mathrm{O}_{8}$ counterparts of the $\mathrm{YBa}_{2} \mathrm{Cu}_{3} \mathrm{O}_{7-\delta}$ system, it corresponds to the temperature at which the leading edge gap found in ARPES experiments for quasiparticles near $(\pi, 0)$ becomes fully open, effectively gapping that portion of the quasiparticle Fermi surface. To a first approximation, on making use of the experimental results for optimally and underdoped $\mathrm{YBa}_{2} \mathrm{Cu}_{3} \mathrm{O}_{7-\delta}$ materials one finds that

$$
T^{*} \approx \frac{1}{3} T_{\mathrm{cr}}
$$

The superconducting $T_{c}$ in Fig. 1 1 is obtained using the empirical relation 138

$$
x=0.16 \pm 0.11 \sqrt{1-\frac{T c}{T_{c}^{\max }}}
$$

where $x$ is the doping level, and $T_{c}^{\max }$ is the maximal transition temperature for a given class of materials. The location of $T_{\text {cr }}$ can well be fitted by another empirical relation.

$$
T_{\mathrm{cr}} \approx 1250 \mathrm{~K}\left(1-\frac{\mathrm{x}}{\mathrm{x}_{\mathrm{cr}}}\right),
$$

where $x_{\mathrm{cr}} \approx 0.19$. Similar expressions are found for the $\mathrm{La}_{2-x} \mathrm{Sr}_{x} \mathrm{CuO}_{4}$ and $\mathrm{Bi}_{2} \mathrm{Sr}_{2} \mathrm{CaCu}_{4} \mathrm{O}_{8}$ materials. This expression for $T_{\text {cr }}$ is, in both its magnitude and doping dependence, close to the pseudogap temperature obtained by Loram and his collaborator 137 from an analysis of specific heat experiments. A remarkable result of this purely phenomenological analysis is that the crossover temperature $T_{\text {cr }}$ extrapolates at zero doping to the known value of the antiferromagnetic super-exchange interaction $J$.

The fit to $T_{\text {cr }}$ by Eq. 104 raises the issue of whether $T^{*}$ and $T_{\mathrm{cr}}$ are independent of $T_{c}$ and would extrapolate to the origin at a doping level $x=x_{\mathrm{cr}}$ if superconductivity was absent. The system then would have an additional quantum critical point at $x=x_{\text {cr }}$ with a new kind of ordered state for $x<x_{\mathrm{cr}}$. This issue is currently open and is a subject of active research. Support for a phase diagram with an additional quantum critical point at $x=x_{\text {cr }}$ comes from the work of Loram , Tallon, and their collaborators 137 , 138 , who have proposed such behavior based on a detailed analysis of their specific heat experiments on underdoped and overdoped systems. Moreover, as Loram Tanner, Panagopoulos and others have emphasized 137 139, in the superconducting state of the low-doping side of $T_{\text {cr }}$ one has "weak" superconducting behavior, with a superfluid density $\rho_{s}$ decreasing with decreasing doping, while on the high doping side one has a "conventional" superconductivity, and a value of $\rho_{s}$ that is nearly independent of the doping concentration. Further support for the idea of an additional 
quantum-critical point comes from the well established fact that optimally doped cuprates are the ones for which the extension of the linear resistivity to $T=0$ yields very small residual resistivity, and from the experiments of Refs. 214,215 which, we recall, show that in the absence of superconductivity the linear temperature dependence of the resistivity extends to lower $T$ indicating that at some doping the resistivity can be linear down to $T=0$. As Laughlin et al.209 have emphasized, the presence of a quantum critical point with a large domain of influence, together with superconductivity, serves to conceal the nature of the non-superconducting ground states on either side of the quantum critical point. One might hope that ARPES experiments near optimal doping would distinguish between a quantum critical behavior with quantum critical point at around optimal doping and a spin fermion scenario with antiferromagnetic quantum critical point at considerably smaller doping concentration. However, a recent analysis of Haslinger et al.121 showed that fits to current experiments with either model is possible and requires in both cases the introduction of a large temperature and frequency independent scattering rate, as noted earlier.

The variety of experimental results for the pseudogap allows one to understand it phenomenologically, without invoking a particular microscopic mechanism. First, as $T^{*}$ and $T_{\text {cr }}$ scale with each other, it is natural to attribute both $T^{*}$ and $T_{\text {cr }}$ to different aspects of the same physical phenomenon which begins at $T_{\mathrm{cr}}$ and gains full strength at $T^{*}$. This idea is fully consistent with NMR data which show the onset of changes in $T_{1} T$ at $T_{c r}$, which eventually give rise to a sign change of the temperature derivative of ${ }^{63} T_{1} T$ at $T^{*}$. Second, ARPES data on the leading edge gap clearly demonstrate that the pseudogap physics below $T^{*}$ is associated with the redistribution of the spectral weight for hot quasiparticles; quasiparticles near the nodes are almost unaffected by the development of the pseudogap. In the ARPES literature, this effect is described as a progressive development of the arcs of the Fermi surface centered around nodal points. The evolution of the full Fermi surface into the arcs begins at around $T^{*}$, and at $T_{c}$ the whole Fermi surface becomes gapped. The "gapping" of hot fermions obviously affects NMR relaxation rates dominated by momenta near $\mathbf{Q}$ (such as ${ }^{63} \mathrm{Cu} T_{1} T$ ) as a spectral weight transfer would lead to a reduction of a desyrate of a spin fluctuation into a particle-hole pain 50,98 NMR experiments by Curro et al.140 and Haase et al.141 show that this is indeed the case. The gapping of hot quasiparticles should also lead to a temperature-dependent reduction in the uniform magnetic susceptibility 143 .

The phenomenological description is of course not enough as it leaves open the key question, namely what causes the spectral weight transfer for hot quasiparticles. We now discuss how the experimental phase diagram fits into the spin-fluctuation scenario.

First of all a general phase diagram based on spin fluctuation approach should distinguish between weakly and strongly antiferromagnetic materials. Weakly antiferromagnetic materials are those to the right of $T_{\text {cr }}$ for which the dimensionless coupling constant $\lambda$ is smaller than unity, which corresponds to a correlation length smaller than a few lattice constants. For these materials, the normal state is a renormalized Fermi liquid, the nearly antiferromagnetic Fermi liquid, and $T_{c}$ signals a transition to a BCS-like superconducting state with a $d_{x^{2}-y^{2}}$ order parameter. For strongly antiferromagnetic materials, on the other hand, the dimensionless coupling constant $\lambda \geq 1$ and $\xi \geq 2 a$. In this situation, the normal state behavior deviates from a Fermi liquid already at comparatively small $\omega$ and $T$ although at the lowest frequencies the system still would display a Fermi liquid behavior if indeed one could extend the normal state down to $T=0$. For these systems, we also know that the pairing instability temperature $T_{\text {cr }}$ increases with decreasing doping and for large enough coupling saturates at a value comparable to the magnetic $J$. Applying this to the experimental phase diagram, we see that optimally doped materials are at the borderline between being weakly and strongly antiferromagnetic: on the one hand the dimensionless coupling is already not small, on the other hand, the pseudogap phase extends at best over a $T$ range which is only a fraction of $T_{\mathrm{cr}}$.

As we have noted above, these results of the spinfermion model make it a strong candidate for the microscopic description of the pseudogap phase: $T_{\text {cr }}$ saturates $T_{\text {cr }}$ saturates at a finite value at the magnetic transition; for $\lambda \geq 1$, the pairing involves non-Fermi liquid fermions; at $T=0$ there are two distinct energy scales in the problem, a fermionic gap $\Delta \propto T_{\text {cr }}$ and a bosonic gap $\Delta_{s} \propto T_{\text {cr }} \lambda^{-1} \ll T_{\text {cr. }}$. The central issue is whether the pairing of incoherent fermions only gradually changes the fermionic self-energy, or whether it creates a feedback on fermions which immediately gives rise to a coherent quasiparticle behavior at the lowest frequencies, as happens in dirty superconductors where $\Sigma(\omega)=i \gamma$ in the normal state transforms below $T_{c}$ into a mass renormalization at the smallest $\omega$

$$
\Sigma(\omega)=i \gamma \frac{\omega}{\left(\omega^{2}-\Delta^{2}\right)^{1 / 2}} \simeq \gamma \frac{\omega}{\Delta}+O\left(\omega^{3}\right) .
$$

If the feedback is gradual, then the pairing creates bound states of incoherent fermions with $S=0$. In this situation, there is a reduction in the density of states below $T_{\text {cr }}$, but a full superconducting gap does not develop until a smaller temperature, $T_{c}$. A simple toy model which describes this physics would be ond 50 in which fermions in the normal state display a quantum critical behavior with $\tilde{\Sigma}(\omega) \approx \Sigma(\omega)=(i \omega \bar{\omega})^{1 / 2}$, and pairing creates a nonzero flat pairing vertex $\Phi$ but does not affect $\Sigma(\omega)$. In this situation, the fermionic propagator acquires a gap at a finite but imaginary frequency:

$$
G_{\mathbf{k}}(\omega) \propto \frac{\sqrt{i \omega \bar{\omega}}+\epsilon_{\mathbf{k}}}{i \omega-E_{\mathbf{k}}}
$$

where 


$$
E_{\mathbf{k}}=\left(\Phi^{2}+\epsilon_{\mathbf{k}}^{2}\right) / \bar{\omega} .
$$

The spectral function at $\mathbf{k}=\mathbf{k}_{F}$ and the fermionic DOS both have broad maxima at $\omega=\Delta=\Phi^{2} / \bar{\omega}$, but the spectral weight is finite at any finite $\omega$, although reduced at low frequencies. Within this model, the transition to the true superconducting state can be understood as a rotation of the pole in Eq. (106) from the imaginary to the real frequency axis such that at the lowest frequencies the $i \omega$ term becomes purely real. The frequency up to which $N(\omega)=0$ then give an estimate for the actual $T_{c}$.

It is unclear to what extent the results of this toy model reflect the physics of the spin-fermion model below $T_{\mathrm{cr}}$. Without elaborating on this subject of current research we mention that as long as an Eliashberg approach is justified, phase fluctuations of the superconducting order parameter cannot substantially reduce $T_{c}$ compared to $T_{\mathrm{cr}}$. Behavior different from that in dirty superconductors could emerge only if longitudinal fluctuations of the superconducting order parameter are soft and able to destroy the superconduting coherence at $T_{c} \ll T_{\text {cr }}$. We have both numerical 102 and analytical 229 evidence that such degeneracy does exist in the limit $\lambda=\infty$. Still, this subject is far from being fully understood and clearly requires further study.

Another subtle issue is whether the spin-fermion model displays quantum critical behavior at $x=x_{c r}$. Physically, this would imply that the pairing of incoherent fermions at $T_{\mathrm{cr}}$ and the pairing of coherent fermions at $T_{c}$ are uncorrelated phenomena - the first gives rise only to the pseudogap, while the latter yields BCS-type superconductivity. Since the pairing of incoherent fermions is not a perturbative phenomenon and requires the interaction to exceed a threshold value 22 , the pairing of incoherent fermions involves only quasiparticles in some finite region around a hot spot and would form a dome on top of a magnetic quantum critical point and vanish at a finite $x$. It is not clear how well one can separate coherent and incoherent pairings.

Another issue related to the possible explanation of the pseudogap within the spin-fermion model is whether one can smoothly interpolate between the limit $\bar{g} \ll W$ ( $W$ is the fermionic bandwidth), where one can perform calculations in a controlled fashion, and $\bar{g} \gg W$ where Mott physics become relevant. In essence the issue is whether or not there is a qualitative difference between limits in which the effective interaction is either much larger or much smaller than the fermionic bandwidth. In the latter case, it is appealing to conjecture that the pseudogap is associated with the fact that it is difficult for hot quasiparticles to be both itinerant and localized. $T_{\text {cr }}$ then would mark the onset of insulating behavior associated with such localization, and the pseudogap phase would represent a kind of partial Mott insulator. A sign that these two limits may describe some aspects of the basic physics similarly is that, as shown earlier, $T_{\text {cr }}$ scales with $\bar{g}$ for $\bar{g}<W$, but crosses over to $W^{2} / \bar{g} \propto J$ for
$\bar{g}>W$. At the same time, the limit $\bar{g}>W$ is probably more rich than the small $\bar{g}$ limit as the physics associated with the localization in the Mott insulator is not included into our analysis. We speculate that due to this Mott physics, bound singlet pairs of fermions that emerge below $T_{\text {cr }}$ could order for example in columnar fashion as suggested by Sachdev and collaborator $\$ 230$ who arrived at a columnar phase by studying weakly doped Mott antiferromagnets. This ordering in turn would imply that $T_{\text {cr }}$ is a true phase transition line below which $Z_{4}$ symmetry is broken. Columnar ordering also opens a link between our approach and the approaches which depart from Mott insulator at half-filling. In particular, columnar ordering of bound electron pairs naturally leads to stripe physics 230 . An alternative pessibility is that singlet pairs remain spatially disordered 218 ,22. In any event, the role of localization effects certainly increases as the system approaches half-filling. Whether they remain strong near optimal doping in the normal state is a subject of debate, but still, strong localization effects should reduce the number of low energy carriers and therefore change the volume of the Fermi surface or increase their mass. ARPES experiments on the other hand indicate that in the normal phase, the Fermi surface is large and obeys Luttinger's theorem without dramatic mass renormalizations. We therefore believe that near optimal doping localization effects are at best moderate.

We conclude this discussion of the phase diagram by mentioning two alternative scenarios for the pseudogap and anomalous normal state properties. The first scenario, pioneered by P.W Anderson 218, X.-G. Wen and P. A. Lee 219 and other 220227 and later modified chiefly by M.P.A. Fisher and T. Senthil228, assumes spin-charge separation at half-filling and explains the whole phase diagram as a result of weak doping of a Mott insulator. A second scenario, on the contrary, assumes that one can understand the phase-diagram within mean-field theory; as Chakravarty et al. 92 have proposed the pseudogap might then be a new protected state of matter that is the result of the breaking of a hidden symmetry.

\section{CONCLUSIONS}

In this Chapter we have demonstrated that superconducting pairing mediated by the exchange of spin fluctuations is a viable alternative to conventional phononmediated pairing. We discussed in detail the normal state properties, the pairing instability and the superconducting behavior of a material near an antiferromagnetic instability, when the dominant interaction between quasiparticles is of electronic origin and, at energies much smaller than the fermionic bandwidth, can be viewed as being due to the emission and absorption of a collective, soft spin degree of freedom. We argued that the spinfluctuation exchange yields an attraction in the $d_{x^{2}-y^{2}}$ channel in agreement with what nearly all researchers 
now believe is the pairing symmetry in the cuprates.

We demonstrated that the physics is qualitatively different depending on whether or not Fermi surface geometry allows a process in which a collective mode decays into a particle and a hole. For this to be possible, the Fermi surface should contain hot spots. We focused on the case in which the Fermi surface does contain hot spots (as the photoemission experiments in cuprates indicate) and showed that spin fluctuations are then overdamped and that their diffusive dynamics should be analyzed in a consistent manner with the low energy dynamics of the fermions. We further argued that contrary to naive expectations, this case is better for $d$-wave pairing than one in which spin fluctuations are propagating, magnonlike quasiparticles.

We showed that the low-energy theory for fermions interacting with overdamped collective spin excitations is universal, independent of the details of the underlying lattice Hamiltonian and is characterized by only two input parameters: the dimensionless coupling constant, $\lambda$, and an overall energy scale, $\bar{\omega}$, proportional to the effective spin-fermion interaction $\bar{g}$. In so doing, we have developed the microscopic justification for the NAFL. The coupling constant, $\lambda$, scales with the magnetic correlation length, so that close enough to a magnetic transition, the system falls into a strong coupling regime. A universal description is valid if $\bar{g}$ is smaller than the fermionic bandwidth $W$ (that would correspond to a weak coupling limit if the system was far away from a magnetic instability). In the opposite limit which we did not discuss in detail, lattice effects become important, and the universality is lost.

At sufficiently low temperatures and energies, the nearly antiferromagnetic Fermi liquid (NAFL) is a Fermi liquid quantum protectorate, according to Landau's criterion-that one can obtain a one-to-one correspondence between the low-lying states of a Fermi gas and the Fermi liquid, as though the particle interaction was turned on adiabatically 134. However, because of the closeness to an antiferromagnetic instability, it is an unconventional Fermi liquid, in that the characteristic energy above which this description is no longer valid is not the Fermi energy, but is the much much lower spinfluctuation energy $\omega_{\text {sf }}=\bar{\omega} /\left(4 \lambda^{2}\right)$ that is typically two orders of magnitude smaller than the Fermi energy. For energies (or temperatures) between $\omega_{\text {sf }}$ and the Fermi energy, we have seen using the spin-fermion model that the system behavior is again universal and depends only on a very limited number of phenomenological parameters. So in this sense, the behavior of an NAFL at energies and temperatures above $\omega_{\text {sf }}$ is also protected, and one finds, in the NAFL, two distinct protected states of matter, depending on the energy or temperature one encounters.

We compared in detail the spin fluctuation approach with the Eliashberg approach to phonon superconductors and showed that despite the absence of the small electron to ionic mass ratio that justified Eliashberg theory for phonons, an Eliashberg-type approach to the spin- fermion model is still justified, but only at strong coupling $\lambda \geq 1$.

We showed that at large $\lambda$, there are two distinct energy scales for the normal state problem: $\bar{\omega}=A \bar{g}$, $A=O(1)$, which at $\bar{g} \sim W$ is of the order of (even though numerically smaller by about an order of magnitude than) the Fermi energy, and a much smaller $\omega_{\text {sf }}=\bar{\omega} /\left(4 \lambda^{2}\right) \ll \bar{\omega}$. Conventional Fermi liquid behavior with $\Sigma^{\prime \prime} \propto \omega^{2}+(\pi T)^{2}$ and an almost temperature independent static spin susceptibility exists only at frequencies and temperatures smaller that $\omega_{\mathrm{sf}}$. At frequencies between $\omega_{\text {sf }}$ and $\bar{\omega}$, the system crosses over into a regime in which spin susceptibility is diffusive and $\Sigma^{\prime \prime} \propto \omega^{\alpha}$. We found that $\alpha=\frac{1}{2}$, while the behavior in the crossover region from Fermi liquid to non Fermi liquid behavior resembles a linear in frequency dependence. In the diffusive regime, both spin and fermionic propagators become independent of $\lambda$, i.e., the system displays magnetic quantum-critical behavior. Finally, at $\omega>\bar{\omega}$, the selfenergy gets smaller than the bare $\omega$, although $\Sigma^{\prime \prime} \propto \omega^{1 / 2}$ still holds.

We next argued that the existence of the two distinct energy scales has a strong impact on the pairing problem: the pairing instability temperature $T_{\text {cr }}$ is predominantly determined by incoherent, non-Fermi liquid fermions with energies between $\omega_{\text {sf }}$ and $\bar{\omega}$. We then considered the superconducting state, found the pairing gap $\Delta \propto T_{\text {cr }}\left(2 \Delta / T_{\text {cr }} \sim 4\right.$ for $\left.\lambda \gg 1\right)$, and argued that one of the signatures of the spin-fluctuation mechanism is the change of the low-frequency spin dynamics from relaxational to propagating due to the feedback from the pairing. This give rise to the emergence of the resonance peak in the spin propagator at a frequency $\Delta_{s}$ which at large $\lambda$ scales as $\Delta_{s} \sim 1.5 T_{\mathrm{cr}} / \lambda$. For smaller $\lambda$, the resonance frequency goes up but always remain smaller than $2 \Delta$. This restriction (not found for phonon superconductors) is a consequence of feedback between fermionic and bosonic dynamics.

We discussed how the emergence of the resonance mode affects fermionic properties and identified several "fingerprints" of the strong coupling spin-fluctuation scenario. These include, but are not limited to, the peak/dip/hump in the spectral function, the nearabsence of the dispersion of the quasiparticle peak, the peak/dip features in the SIN and SIS tunneling conductances, and in the second derivative of the optical conductivity.

Finally, we compared the theoretical results with the normal state data for near optimally-doped cuprates, and found that a large number of experimental results can be understood within the spin-fluctuation scenario. In particular, we argued that the tunneling, photoemission and optical data for the superconducting state display the expected "fingerprints" of the spin-fluctuation mechanism for superconductivity in the cuprates.

The spin-fluctuation theory should be equally applicable to quasi two dimensional organic superconductors or compounds of the family $\mathrm{CeXIn}_{5}$ with $\mathrm{X}=\mathrm{Co}, \mathrm{Rh}$, 
Ir, if the Fermi surface of these systems possesses hot spots. A detailed experimental investigation of spectroscopic properties of these systems in the superconducting state will provide important clues on whether they are indeed magnetically mediated superconductors.

Despite the successful identification of the "fingerprints" of spin mediated pairing in the cuprates, we listed in Section 6.4 some properties of these materials which we have not explained within the spin-fluctuation scenario. Perhaps the most important remaining question is to what extent the spin-fluctuation theory explains the pseudogap physics in underdoped cuprates. The existence of the two distinct energy scales in the spin-fermion model and the presence of fermionic incoherence in the normal state make this model a good candidate model for the pseudogap. The plausible argument here is that while the pairing of the incoherent fermions will create spin singlets, fermions will still behave incoherently and hence not superconduct until a smaller $T_{c}$ is reached when feedback from the pairing restores fermionic coherence. Particularly relevant here is whether or not there exists an additional critical point on the phase diagram at around $x=0.19$. This critical point emerges in the spin-fluctuation theory if the pairing of incoherent fermions from frequencies above $\omega_{s f}$ and the pairing of coherent fermions from frequencies below $\omega_{\mathrm{sf}}$ are separate phenomena (the first then definitely gives rise to a pseudogap physics while the second yields a true superconductivity). The pairing of incoherent fermions is a threshold phenomenon and thus occurs at $T_{\mathrm{cr}}(x)$. We still however need to understand whether incoherent pairing and coherent pairing can be totally separated from each other.

Another unresolved issue is to what extent the fact that the parent compounds of the cuprates $\left(\mathrm{La}_{2} \mathrm{CuO}_{4}\right.$ or $\mathrm{YBa}_{2} \mathrm{Cu}_{3} \mathrm{O}_{6}$ ) are Mott insulators and nearest-neighbor Heisenberg antiferromagnets affects the behavior of doped materials. For the spin-fermion model, this question could be reformulated as whether the limit $\bar{g} \ll W$, in which we can separate low and high energies and perform controlled calculations, and the limit $\bar{g} \gg W$, are only quantitatively different or are qualitatively different. An encouraging sign that the two limits may not be very different is the result that $T_{\text {cr }}$ smoothly interpolates between $O(\bar{g})$ for $\bar{g} \ll W$ to $O\left(W^{2} / \bar{g}\right)$ for $\bar{g} \gg W$, in the latter case $T_{\text {cr }}$ becomes of order of a magnetic exchange integral $J$. On the other hand, the localization effects which accompany a transition to a Mott insulator are not included in the theory presented here. In particular, we cannot predict what would happen with singlet pairs below $T_{\mathrm{cr}}$, if it indeed is the onset of the pseudogap - whether they remain disorderedor form columnar stripes as Sachdev and collaborator\$230 suggested.

The enduring presence and richness of unsolved problems in the field of unconventional superconductivity makes us optimistic that we will continue to see unexpected experimental observations and new, creative theoretical concepts in this field of research. We have enjoyed over a number of years our respective collaborations on the spin fluctuation approach because of its clarity and ability to make falsifiable predictions for experiment. We hope that the readers of this Chapter will share our excitement for this approach to magnetically mediated superconductivity.

\section{ACKNOWLEDGMENTS}

It is our pleasure to thank our immediate collaborators Artem Abanov, Victor Barzykin, Alexander Finkelstein, Rob Haslinger, Philippe Monthoux and Branko Stojkovic for stimulating discussions on essentially all aspects of the spin fluctuation approach presented here. We are also thankful to E. Abrahams, A. A. Abrikosov, G. Aeppli, B. L. Altshuler, N. Andrei, A. Balatsky, D. Basov, K. H. Bennemann, G. Blumberg, J. Brinckmann, J.C. Campuzano, P. Chandra, P. Coleman, E. Dagotto, J. C. Davis, E. Demler, M. P. A. Fisher, A. Georges, L.P. Gor'kov, M. Grilli, L. Ioffe, P. Johnson, R. Joynt, A. Kaminski, B. Keimer, D. Khveschenko, S. Kivelson, G. Kotliar, R. Laughlin, M. Lavagna, P. A. Lee, G. Lonzarich, H. von Löhneysen, A. Millis, H. Monien, D. K. Morr, M. Norman, C. Pépin, A. Rosch, S. Sachdev, D. Scalapino, J. R. Schrieffer, T. Senthil, Q. Si, C. P. Slichter, S. Sondhi, Z. Tesanovic, O. Tchernyshyov, A.Tsvelik, J. Tu, C. Varma, P. G. Wolynes, A. Yazdani, J. Zasadzinski and S.-C. Zhang for useful conversations. We further wish to thank Ar. Abanov, D. Basov, J. C. Davis and J. Tu for sharing their unpublished results with us.

The research which led to this review was supported in part by NSF DMR-9979749 ( A. Ch.), by the Institute for Complex Adaptive Matter, an independent unit of the University of California, by Project DR 200153 on Emergent Properties of Correlated Electron Superconductors and general DOE support to Los Alamos National Laboratory (D. P.), and by the Ames Laboratory, operated for the U.S. Department of Energy by Iowa State University under contract No. W-7405-Eng-82 (J.S).

\footnotetext{
${ }^{1}$ See for example: Proceedings of Materials and Mechanisms of Superconductivity: High Temperature Superconductors VI in Physica C (Amsterdam), 341-348 (2000).

${ }^{2}$ J. Bardeen and D. Pines, Phys. Rev. 99, 1140-1150 (1955).

${ }^{3}$ J. Bardeen, L. Cooper, J. R. Schrieffer, Phys. Rev. 108, 1175-1204 (1957).

${ }^{4}$ W. L. McMillan and J. M. Rowell, Tunneling and Strong Coupling Superconductivity, in Superconductivity, Vol. 1, p. 561, Ed. Parks, Marull, Dekker Inc. N.Y. (1969).

${ }^{5}$ D. J. Scalapino, The electron-phonon interaction and strong coupling superconductors, in Superconductivity, in
} 
Superconductivity, Vol. 1, p. 449, Ed. R. D. Parks, Dekker Inc. N.Y. 1969.

${ }^{6}$ E. Maxwell, Phys. Rev. 78, 477 (1950).

${ }^{7}$ A. Reynolds, B. Serin, W. H. Wright, and L. B. Nesbit, Phys. Rev. 78, 487 (1950).

${ }^{8}$ G. M. Eliashberg, Sov. Phys. JETP 11, 696 (1960).

${ }^{9}$ L. D. Landau, Sov. Phys. JETP 3, 920-925 (1957).

${ }^{10}$ R. Laughlin and D. Pines, Proceedings of the National Academy of Science, 97, 28-31 (2000).

${ }^{11}$ W. Kohn and J. M. Luttinger, Phys. Rev. Lett. 15, 524 (1965).

12 A. J. Leggett, Rev. Mod. Phys. 47, 331 (1975).

${ }^{13}$ D. Vollhardt, and P. Wölfle, The Superfluid Phases of Helium 3, Taylor and Francis, London 1990.

${ }^{14}$ G. E. Volovik, Exotic Properties of ${ }^{3} \mathrm{He}$, World Scientific, (1991).

15 V.J. Emery, J. Phys. (Paris) Colloq. 44, C3-977 (1983); Synth. Met. 13, 21 (1986).

16 J. E. Hirsch, Phys. Rev. Lett. 54, 1317 (1985).

${ }^{17}$ K. Miyake, S. Schmitt-Rink, and C. M. Varma, Phys. Rev. B 34, 6554 (1986).

${ }^{18}$ D. J. Scalapino, E. Loh, Jr. and J. E. Hirsch, Phys. Rev. B 34, 8190 (1986); 356694 (1987).

19 N. E. Bickers, D. J. Scalapino, and R. T. Scalettar, Int. J. Mod. Phys B 1, 687 (1987).

${ }^{20}$ A. J. Millis, S. Sachdev, and C. M. Varma, Phys. Rev. B 37, 4975 (1988).

${ }^{21}$ G. Kotliar, Phys. Rev. B 37, 3664 (1988).

22 P.W. Anderson, S. Chakravarty, S. Strong and A. Sudbo, Science 261, 337 (1993).

${ }^{23} \mathrm{P}$. W. Anderson, The Theory of Superconductivity in the High-Tc Cuprate Superconductors, Princeton University Press, Princeton, NJ (1997).

${ }^{24}$ A. J. Leggett, Proceedings of the National Academy of Science, 96, 8365-8372 (2000).

${ }^{25}$ D. A. Wollmann, D. J. Van Harlingen, W. C. Lee, D. M. Ginsberg, and A. J. Leggett, Phys. Rev. Lett. 71, 2134 (1993).

${ }^{26}$ C. C. Tsuei, J. R. Kirtley, C. C. Chi, Lock See Yu-Jahnes, A. Gupta, T. Shaw, J. Z. Sun, and M. B. Ketchen, Phys. Rev. Lett. 73, 593 (1994).

${ }^{27}$ D. A. Brawner and H. R. Ott, Phys. Rev. B 50, 6530 (1994).

${ }^{28}$ R. Hlubina and T. M. Rice, Phys. Rev. B51, 9253 (1995); 52, 13043 (1995).

${ }^{29}$ T. Moriya, Y. Takahashi and K. Ueda: J. Phys. Soc. Jpn. 59 (1990) 2905.

30 T. Moriya, Y. Takahashi and K. Ueda, Physica C 185189, 114, (1991)

${ }^{31}$ D. Pines, Physica B 163, 78 (1990).

${ }^{32}$ P. Monthoux, A. V. Balatsky and D. Pines, Phys. Rev. Lett.67, 3448 (1991).

${ }^{33}$ C. P. Slichter, in Strongly Correlated Electron Systems, ed. K. S. Bedell et al. (Addison-Wesley, Reading, MA,1994).

${ }^{34}$ A. J. Millis, H. Monien, and D. Pines, Phys. Rev. B 42, 167 (1990).

${ }^{35}$ K. Ueda, T. Moriya and Y. Takahashi, in Electronic Properties and Mechanisms of High-Tc superconductors (Tsukuba Symposium, 1991), eds. T. Oguchi, et al. (North Holland, Amsterdam, 1992) 145; J. Phys, Chem. Solids 53
1515 (1992).

${ }^{36}$ P. Monthoux and D. Pines: Phys. Rev. Lett. 69, 961 (1992).

${ }^{37}$ P. Monthoux and D. Pines, Phys. Rev. B 48, 6069 (1993).

${ }^{38}$ P. Monthoux and D. Pines, Phys. Rev. B 50, 16015 (1994).

${ }^{39}$ High Temperature Superconductivity, Eds.K. S. Bedell, D. Coffey, D. Melzer, D. Pines, and J. R. Schrieffer, AddisonWesley, Reading, MA (1990).

${ }^{40}$ S. E. Barrett, D. J. Durand, C. H. Pennington, C. P. Slichter, T. A. Friedmann, J. P. Rice, and D. M. Ginsberg, Phys. Rev. B 41, 6283-6296 (1990).

${ }^{41}$ M. Takigawa, P. C. Hammel, R. H. Heffner, and Z. Fisk, Phys. Rev. B 39, 7371-7374 (1989).

${ }^{42}$ H. Monien and D. Pines, Phys. Rev. B 41, 6297 (1990).

${ }^{43}$ R. E. Walstedt, W. W. Warren, Jr., R. F. Bell, G. F. Brennert, G. P. Espinosa, J. P. Remeika, R. J. Cava, and E. A. Rietman, Phys. Rev. B 36, 5727-5730 (1987).

${ }^{44}$ W. W. Warren, Jr., R. E. Walstedt, G. F. Brennert, G. P. Espinosa, and J. P. Remeika, Phys. Rev. Lett.59, 18601863 (1987)

${ }^{45}$ Z. X. Shen, D. S. Dessau, B. O. Wells, D. M. King, W. E. Spicer, A. J. Arko, D. Marshall, L. W. Lombardo, A. Kapitulnik, P. Dickinson, S. Doniach, J. DiCarlo, T. Loeser, and C. H. Park, Phys. Rev. Lett. 70 1553-6 (1993).

${ }^{46}$ W. N. Hardy, D. A. Bonn, D. C. Morgan, Ruixing Liang, and Kuan Zhang, Phys. Rev. Lett. 70, 3999-4002 (1993).

${ }^{47}$ J. A. Martindale, S. E. Barrett, K. E. O'Hara, C. P. Slichter, W. C. Lee, and D. M. Ginsberg, Phys. Rev. B 47, 9155-9157 (1993).

${ }^{48}$ N. Bulut and D. J. Scalapino, Phys. Rev. Lett.68, 706-709 (1992).

49 D. Thelen, D. Pines and Jian Ping Lu, Phys. Rev. B 47, 9151-9154 (1993).

${ }^{50}$ A. V. Chubukov, Phys. Rev. B 52, R3840 (1995); Europhys. Lett. 44, 655 (1998).

${ }^{51}$ A. Abanov, A. V. Chubukov, J. Schmalian, condmat/0107421, to appear in Advances in Physics.

J2 J. Schmalian, Phys. Rev. Lett. 81, 4232 (1998).

${ }^{53}$ H. Kino and H. Kontani, J. Phys. Soc. Japan 67, 3691 (1998).

${ }^{54}$ H. Kondo and T. Moriya, J. Phys. Soc. Japan 67, 3695 (1998).

${ }^{55}$ K. Kuroki and H. Aoki, Phys. Rev. B 60, 3060 (1999).

${ }^{56}$ S. M. De Soto, C. P. Slichter, A. M. Kini, H. H. Wang, U. Geiser, and J. M. Williams, Phys. Rev. B52, 10364 (1995).

${ }^{57}$ H. Mayaffre, P. Wzietek, D. Jerome, C. Lenoir, and P. Batail , Phys. Rev. Lett. 75, 4122 (1995).

${ }^{58}$ K. Kanoda, K. Miyagawa, A. Kawamoto, Y. Nakazawa, Phys. Rev. B 54, 76 (1996).

${ }^{59}$ S. Belin, K. Behnia, and A. Deluzet, Phys. Rev. Lett. 81, 4728 (1998).

${ }^{60}$ K. Izawa, H. Yamaguchi, T. Sasaki, Y. Matsuda, condmat/0107537 (2001).

${ }^{61}$ J. M. Schrama, E. Rzepniewski, R. S. Edwards, J. Singleton, A. Ardavan, M. Kurmoo, and P. Day, Phys. Rev. Lett. 83, 3041 (1999).

${ }^{62}$ T. Arai, K. Ichimura, K. Nomura, Takasaki, J. Yamada, S. Nakatsuji, and H. Anzai, Phys. Rev. B 63, 104518 (2001).

${ }^{63}$ M. Lang, N. Toyota, T. Sasaki, and H. Sato, Phys. Rev. 
Lett. 69, 1443 (1992)

${ }^{64}$ J. Müller, M. Lang, R. Helfrich, F. Steglich, T. Sasaki, cond-mat/0109030.

65 S. Lefebvre, P. Wzietek, S. Brown, C. Bourbonnais, D. Jerome, C. Meziere, M. Fourmigue, P. Batail, Phys. Rev. Lett. 85, 5420 (2000).

${ }^{66}$ F. Steglich, J. Aarts, C. D. Bredl, W. Lieke, D. Meschede, W. Franz, and H. Schäfer, Phys. Rev. Lett. 43, 1892 (1979).

${ }^{67}$ N. D. Mathur, F. M. Grosche, S. R. Julian, I. R. Walker, D. M. Freye, R. K. W. Haselwimmer, G. G. Lonzarich, Nature (London) 394, 39-43 (1998).

${ }^{68}$ C. Petrovic, P. G. Pagliuso, M. F. Hundley, R. Movshovich, J. L. Sarrao, J. D. Thompson, Z. Fisk, and P. Monthoux , J. Phys. Condens. Mat. 13, L337 (2001); C. Petrovic, R. Movshovich, M. Jaime, P. G. Pagliuso, M. F. Hundley, J. L. Sarrao, Z. Fisk, and J. D. Thompson, Europhys. Lett.53 354 (2001); H. Hegger, C. Petrovic, E. G. Moshopoulou, M. F. Hundley, J. L. Sarrao, Z. Fisk, and J. D. Thompson , Phys. Rev. Lett. 844986 (2000).

${ }^{69}$ P.G. Pagliuso, R. Movshovich, A.D. Bianchi, M. Nicklas, N.O. Moreno, J.D. Thompson, M.F. Hundley, J.L. Sarrao, Z. Fisk, cond-mat/0107266.

${ }^{70}$ K. Izawa, H. Yamaguchi, Yuji Matsuda, H. Shishido, R. Settai, and Y. Onuki, Phys. Rev. Lett. 87, 057002 (2001).

${ }^{71}$ Y. Maeno, H. Hashimoto, Y. Yoshida. S. Nishizaki, T. Fujita, J. G. Bednorz, and F. Lichtenberg, Nature (London) 372, 532 (1994).

${ }^{72}$ Y. Maeno, T. M. Rice, and M. Sigrist, Physics Today 54, $42(2001)$

73 T.M. Rice and M. Sigrist, J. Phys.: Condens. Matter 7, L643 (1995).

${ }^{74}$ P. Monthoux and G. G. Lonzarich, Phys. Rev. B 63, 054529 (2001)

${ }^{75}$ K. Ishida, H. Mukuda, Y. Kitaoka, K. Asayama, Z. Q. Mao, Y. Mori, and Y. Maeno, Nature 96, 658 (1998).

76 J.A. Duffy, S. M. Hayden, Y. Maeno, Z. Mao, J. Kulda, and G. J. McIntyre, Phys. Rev. Lett. 85, 5412 (2000).

77 J. A. Hertz, Phys. Rev. B 14, 1165 (1976).

78 A. J. Millis, Phys. Rev. B 48, 7183 (1993).

${ }^{79}$ P. Coleman, C. Pepin, Q. Si, R. Ramazashvili, condmat/0105006.

${ }^{80}$ Q. Si, Journ. of Magnetism and Mag. Materials, 226-230, 30 (2001).

${ }^{81}$ F. C. Zhang, T. M. Rice, Phys. Rev. B 37, 3759 (1988).

82 J.R. Schrieffer, X.G. Wen, and S.C. Zhang, Phys. Rev. B39, 11663 (1989).

${ }^{83}$ J. R. Schrieffer, J. Low Temp. Phys. 99, 397 (1995).

${ }^{84}$ A.V. Chubukov and D. Frenkel, Phys. Rev. B 46, 11884 (1992)

85 A.V. Chubukov and D.K. Morr, Phys. Rep.,288, 355 (1997).

${ }^{86}$ S. L. Adler, Phys. Rev. 137, B1022 (1965).

${ }^{87}$ M.Yu. Kuchiev, O. P. Sushkov, Phys. Rev. B. 53443 (1996).

${ }^{88}$ W. L. McMillan, Phys. Rev., 167331 (1968).

${ }^{89}$ H. J. Schulz, Phys. Rev. B 39, 2940 (1989).

90 A.A. Nersesyan, Phys. Lett., A153, 49 (1991); A. Luther and A.A. Nersesyan, Phys. Rev. B 50, 309 (1994) and references therein.
${ }^{91}$ J. B. Marston and I. Affleck, Phys. Rev. B 43, 11538 (1989); B 61, 14773 (2000).

92 S. Chakravarty, R. Laughlin, D. K. Morr, C. Nayak, Phys. Rev. B 63, 094503 (2001).

${ }^{93}$ C. M. Varma, P.B. Littlewood, S. Schmitt-Rink, E. Abrahams, A. E. Ruckenstein, Phys. Rev. B 63, 1996 (1989); Phys. Rev. Lett. 64, 497 (1990).

${ }^{94}$ C. M. Varma, Phys. Rev. Lett. 83, 3538 (1999); Phys. Rev. B 55, 14554 (1997).

${ }^{95}$ C. Castellani, C. DiCastro, and M. Grilli, Z. Phys. B 103, 137 (1997).

${ }^{96}$ M. Vojta, Y. Zhang, and S. Sachdev, Phys. Rev. Lett. 85, $4940(2000)$

${ }^{97}$ D. Pines, Physica C 341-348, 59 (2000).

98 J. Schmalian, D. Pines, B. Stojkovich, Phys. Rev. Lett.80, 3839 (1998).

${ }^{99}$ L. B. Ioffe and A. J. Millis, Uso. Fiz. Nauk. 41, 595 (1998).

100 J. M. Kosterlitz and D. J. Thouless, J. Phys. C 6, 1181 (1973).

${ }^{101}$ L. Kadanoff, Phys. Rev. 132, 2073 (1963).

102 A. Abanov, A. V. Chubukov, J. Schmalian, Europhys. Lett.55 369 (2001).

${ }^{103}$ P. Monthoux and D. J. Scalapino, Phys. Rev. Lett. 72, 1874 (1994).

104 C.-H. Pao and N. E. Bickers, Phys. Rev. Lett. 72, 1870 (1994).

105 T. Dahm, L. Tewordt, Phys. Rev. Lett., 74, 793 (1997).

106 T. Dahm, D. Manske, L. Tewordt, Europhys. Lett., 39, 201 (1997).

107 S. Grabowski, M. Langer, J. Schmalian, and K. H. Bennemann, Europhys. Lett., 34, (1996).

108 M. Langer, J. Schmalian, S. Grabowski, and K. H. Bennemann, Phys. Rev. Lett., 75, 4508 (1995).

${ }^{109}$ A. Abanov, A. V. Chubukov, J. Schmalian, Phys. Rev. B 63, 180510 (2001).

${ }^{110}$ P. Monthoux and D. Pines, J. Phys. Chem. Solids (1995), 56, 1651 .

${ }^{111}$ V. J. Emery and S. A. Kivelson, Nature, London 374, 434 (1995); Phys. Rev. Lett. 74, 3253 (1995).

112 M. Franz and A. J. Millis, Phys. Rev. B 58, 14572 (1998).

113 H.-J. Kwon and A. T. Dorsey, Phys. Rev. B59, 6438 (1999).

114 L.B. Ioffe and A.J. Millis, Science 285, 1241 (1999).

115 M. Franz and Z. Tesanovic, Phys. Rev. Lett. 87, 257003 (2001).

116 R.L. Stratonovich, Dokl. Akad. Nauk SSSR 115, 1097 (1957). [Soviet Phys. Doklady 2, 416 (1958).]

117 J. Hubbard, Phys. Rev. Lett.3, 77 (1959).

118 A. H. Castro Neto and B. Jones, Phys. Rev. B 62, 14975 (2000) (see Appendix A).

119 J. P. Carbotte, Rev. Mod. Phys.62, 1027 (1990), see also the article by F. Marsiglio and J.P. Carbotte in this volume.

${ }^{120}$ H. Won and K. Maki, Phys. Rev. B 49, 1397 (1994); Ye Sun and K. Maki, Phys. Rev. B 51, 6059 (1995).

${ }^{121}$ R. Haslinger, A. Abanov, and A. Chubukov, condmat/0111572, Europhys. Lett., to appear (2002).

${ }^{122}$ Ar. Abanov, A. V. Chubukov and A. M. Finkel'stein, Europhys. Lett., 54, 488 (1999).

${ }^{123}$ R. Roussev and A. J. Millis, Phys. Rev. B 63, 140504 
(2001).

124 G.D. Mahan, Many-Particle Physics, Plenum Press, 1990.

125 A. Migdal, Soviet Physics JETP 7, 996-1001 (1958).

126 S.B. Treiman, R. Jackiw, and D.J. Gross, in Lectures on Current Algebra and its Applications, (Princeton University Press, Princeton, 1972).

${ }^{127}$ M.R. Norman and H. Ding, Phys. Rev. B 57 R11089 (1998).

128 M. R. Norman, Phys. Rev. B 61, 14751 (2000); M. Eschrig and M.R. Norman, Phys. Rev. Lett.85, 3261 (2000).

129 C. Stemmann, C. Pépin, and M. Lavagna, Phys. Rev. B 50, 4075 (1994); D. Z. Liu, Y. Zha, and K. Levin, Phys. Rev. B 75, 4130 (1995); I. Mazin and V. Yakovenko, ibid. 75, 4134 (1995); A. Millis and H. Monien, Phys. Rev. B54, 16172 (1996); N. Bulut and D. Scalapino, Phys. Rev. B 53, 5149 (1996); D. K. Morr and D. Pines, Phys. Rev. Lett.81, 1086 (1998); S. Sachdev and M. Vojta, Physica B 280, 333 (2000); Y.-J. Kao et al., Phys. Rev. B 61, R11898 (2000); F. Onufrieva and P. Pfeuty, cond-mat/9903097.

130 O. Tchernyshyov, M. R. Norman, A. V. Chubukov, Phys. Rev. B 63, 144507 (2001).

131 A. V. Chubukov, B. Janko, O. Tchernyshyov, Phys. Rev. B 63, 180507 (2001)

132 Ar. Abanov, unpublished.

${ }^{133}$ L. D. Landau, E. M. Lifshits and L. P. Pitaevskii, Statistical Physics, Part 2, Pergamon Press (1969).

${ }^{134}$ D. Pines and P. Nozieres, The Theory of Quantum Liquids, W. A. Benjamin, New York (1966).

${ }^{135}$ V. Barzykin and D. Pines, Phys. Rev. B 54, 7567 (1995).

${ }^{136}$ H. Y. Hwang, B. Batlogg, H. Takagi, H. L. Kao, J. Kwo, R. J. Cava, J. J. Krajewski, and W. F. Peck, Jr., Phys. Rev. Lett. 72, 2636 (1994)

137 J. Loram, K. A. Mirza, J. R. Cooper, and W. Y. Liang, Phys. Rev. Lett. 71, 1740 (1993); J.W. Loram, K. A. Mirza, J. R. Cooper, W. Y. Liang, J. M. Wade, J. Supercond., 7, 243 (1994).

138 J. L. Tallon, J. W. Loram, G. V. M. Williams, J. R. Cooper, I. R. Fisher, J. D. Johnson, M. P. Staines, C. Bernhard, Phys. Stat. Sol. (b)215, 531 (1999).

139 C. Panagopoulos, B. D. Rainford, J. R. Cooper, and C. A. Scott, Physica C 341-348, 843 (2000).

${ }^{140}$ N. J. Curro, P. C. Hammel, B. J. Suh, M. Hücker, B. Büchner, U. Ammerahl, and A. Revcolevschi, Phys. Rev. Lett. 85, 642 (2000).

${ }^{141}$ J. Haase, C. P. Slichter, R. Stern, C. T. Milling, D. G. Hinks, J. Supercond. 13, 723 (2000).

142 G. Aeppli, T. E. Mason, S. M. Hayden, H. A. Mook, J. Kulda, Science 278, 1432 (1997).

${ }^{143}$ H. Alloul, P. Mendels, G. Collin, and Ph. Monod, Physica C 156, 355 (1988).

144 J. Schmalian, S. Grabowski, and K. H. Bennemann, Phys. Rev. B, 56 R509 (1997).

145 J. Friedel, J. Phys. Condens. Matter 1, 7757 (1989).

146 See e.g., P. Monthoux, A. Balatsky and D. Pines, Phys. Rev. B 46, 14803 (1992); D.J. Scalapino, Phys. Rep. 250, 329 (1995).

${ }^{147}$ M. R. Norman, H. Ding, J. C. Campuzano, T. Takeuchi, M. Randeria, T. Yokoya, T. Takahashi, T. Mochiku, and K. Kadowaki Phys. Rev. Lett. 79 , 3506 (1997).

148 Z-X. Shen et al, Science 280, 259 (1998).
149 Ar. Abanov, A. V. Chubukov, J.Schmalian, Journal of Electron Spectroscopy and Related Phenomena 117, 129 (2001).

150 S. H. Pan, E. W. Hudson, K. M. Lang, H. Eisaki, S. Uchida, J. C. Davis, Nature, 403, 746 (2000).

151 A.V. Fedorov, T. Valla, and P. D. Johnson, Q. Li, G. D. Gu, N. Koshizuka, Phys. Rev. Lett. 82, 2179 (1999).

152 T. Valla, A. V. Fedorov, P. D. Johnson, B. O. Wells, S. L. Hulbert, Q. Li, G. D. Gu, and N. Koshizuka, Science, 285, 2210 (1999).

${ }^{153}$ A. Kaminski, J. Mesot, H. Fretwell, J. C. Campuzano, M. R. Norman, M. Randeria, H. Ding, T. Sato, T. Takahashi, T. Mochiku, K. Kadowaki, and H. Hoechst, Phys. Rev. Lett. 84, 1788 (2000).

${ }^{154}$ Ch. Renner, B. Revaz, J.-Y. Genoud, K. Kadowaki, and O. Fischer, Phys. Rev. Lett. 80, 149 (1998).

${ }^{155}$ Y. DeWilde, N. Miyakawa, P. Guptasarma, M. Iavarone, L. Ozyuzer, J. F. Zasadzinski, P. Romano, D. G. Hinks, C. Kendziora, G. W. Crabtree, and K. E. Gray, Phys. Rev. Lett. 80, 153 (1998).

156 J. Brinckmann and P.A. Lee, Phys. Rev. Lett. 82, 2915 (1999).

157 J. F. Zasadzinski, L. Ozyuzer, N. Miyakawa, K.E. Gray, D.G. Hinks and C Kendzora, Phys. Rev. Lett. 87, 067005 (2001).

158 A. V. Puchkov et al. J. Phys. Chem. Solids 59, 1907 (1998); D. N. Basov, R. Liang, B. Dabrovski, D. A. Bonn, W. N. Hardy, and T. Timusk, Phys. Rev. Lett. 77, 4090 (1996).

159 A. Chainani, T. Yokoya, T. Kiss, and S. Shin, Phys. Rev. Lett. 85, 1966 (2000), and references therein.

160 J. P. Carbotte, E. Schachinger, D. N. Basov, Nature (London) 401, 354 (1999).

${ }^{161}$ H.F. Fong, B. Keimer, D. Reznik, D. L. Milius, and I. A. Aksay, Phys. Rev. B 54, 6708 (1996); H. F. Fong, P. Bourges, Y. Sidis, L. P. Regnault, J. Bossy, A. Ivanov, L. Milius, I. A. Aksay, and B. Keimer, Phys. Rev. B 61, 14773 (2000).

${ }^{162}$ P. Dai, H. A. Mook, S. M. Hayden, G. Aeppli, T. G. Perring, D. Hunt, and F. Dogan, Science 284, 1344 (1999). P. Dai, H. A. Mook, R. D. Hunt, F. Dogan, Phys. Rev. Lett. 63, 054525 (2001).

${ }^{163}$ H.F. Fong, P. Bourges, Y. Sidis, L.P. Regnault, A. Ivanov, G.D. Gu, N. Koshizuka, and B. Keimer, Nature 398, 588 (1999).

164 T. Holstein, Phys. Rev. 96, 535 (1954); P.B. Allen, Phys. Rev. B 3, 305 (1971).

${ }^{165}$ P. Littlewood and C.M. Varma, Phys. Rev. B 46, 405 (1992).

${ }^{166}$ D. J. Scalapino, J. R. Schrieffer and J. W. Wilkins, Phys. Rev. 148, 263 (1966).

${ }^{167}$ W. Shaw and J.C. Swihart, Phys. Rev. Lett., 20, 1000 (1968).

168 D. Coffey, Phys. Rev. B 42, 6040 (1990).

169 A. Chubukov, Europhys. Lett. 44, 655 (1997).

${ }^{170}$ Ar. Abanov and A. Chubukov, Phys. Rev. Lett., 83, 1652 (1999).

171 Ar. Abanov and A. Chubukov, Phys. Rev. Lett., 84, 5608 (2000).

172 Ar. Abanov and A. Chubukov, Phys. Rev. B 61 ,R9241 
(2000).

173 Ar. Abanov, A. V. Chubukov, N. Gemelke, Phys. Rev. B 61, R787 (2000).

${ }^{174}$ P. Bourges, Y. Sidis, H.F. Fong, B. Keimer, L.P. Regnault, J. Bossy, A.S. Ivanov, D.L. Milius, I.A. Aksay, High Temperature Superconductivity, Edited by S.E. Barnes et al, 207-212, American Institute of Physics, Amsterdam, 1999.

${ }^{175}$ P. Bourges, Y. Sidis, H. F. Fong, L. P. Regnault, J. Bossy, A. Ivanov, and B. Keimer, Science 288, 1234 (2000).

176 J. C. Campuzano, H. Ding, M. R. Norman, H. M. Fretwell, M. Randeria, A. Kaminski, J. Mesot, T. Takeuchi, T. Sato, T. Yokoya, T. Takahashi, T. Mochiku, K. Kadowaki, P. Guptasarma, D. G. Hinks, Z. Konstantinovic, Z. Z. Li, and H. Raffy Phys. Rev. Lett 83, 3709 (1999).

177 see e.g. A. Chubukov, G. Blumberg, and D. Morr, Solid State Comm. 112, 183 (1999).

178 J. R. Schrieffer, Theory of Superconductivity, Benjamin, Reading, Mass. (1966).

179 T.P. Devereaux, D. Einzel, B. Stadlober, R. Hackl, D. H. Leach, and J. J. Neumeier Phys. Rev. Lett. 72, 396 (1994); ibid Phys. Rev. B 54, 12523 (1996).

180 S. M. Quinlan, P. J. Hirschfeld, D. J. Scalapino, Phys. Rev. B 53, 8575 (1996).

${ }^{181}$ R. Haslinger, A. Chubukov, and Ar. Abanov, Phys. Rev. B 63, 020503 (2001)

${ }^{182}$ B. Farnworth and T. Timusk, Phys. Rev. B 10, 2799 (1974)

183 D. A. Bonn, P. Dosanjh, R. Liang, and W. N. Hardy, Phys. Rev. Lett. 68, 2390 (1992).

${ }^{184}$ M. C. Nuss, P. M. Mankiewich, M. L. M'OMalley, and E. H. Westwick, Phys. Rev. Lett. 66, 3305 (1991).

${ }^{185}$ I. Maggio-Aprile, Ch. Renner, A. Erb, E. Walker, and Ø. Fischer, Phys. Rev. Lett.75, 2754 (1995).

${ }^{186}$ N. Miyakawa, J. F. Zasadzinski, L. Ozyuzer, P. Guptasarma, D. G. Hinks, C. Kendziora, and K. E. Gray, Phys. Rev. Lett. 83, 1018 (1999).

${ }^{187}$ B.W. Statt and A. Griffin, Phys. Rev. B 46, 3199 (1992); S.M. Quinlan, D.J. Scalapino and N. Bulut, ibid 49, 1470 (1994).

188 Z-X. Shen and J.R. Schrieffer, Phys. Rev. Lett. 78, 1771 (1997).

189 S. Spielman, D. Parks, J. Orenstein, D. T. Nemeth, F. Ludwig, J. Clarke, P. Merchant, and D. J. Lew, Phys. Rev. Lett. 73, 1537 (1994).

190 J.J. Tu, C.C. Homes, G.D. Gu, D.N. Basov, S.M. Loureiro, R.J. Cava, M. Strongin, cond-mat/0104208.

${ }^{191}$ M.A. Quijada, D. B. Tanner, R. J. Kelley, M. Onellion, H. Berger and G. Margaritondo, Phys. Rev. B 60, 14917 (1999)

192 L. Ioffe and A. I. Larkin, Phys. Rev. B 39, 8988 (1989).

193 P.V. Bogdanov, A. Lanzara, S. A. Kellar, X. J. Zhou, E. D. Lu, W. Zheng, G. Gu, K. Kishio, J. -I. Shimoyama, Z. Hussain, Z. X. Shen, Phys. Rev. Lett. 85, 2581 (2000).

${ }^{194}$ V. Barzykin, D. Pines, A. Sokol, and D. Thelen Phys. Rev. B 49, 1544 (1994)

195 e.g. A. Puchkov, D. Basov, and T. Timusk, J. Phys.: Cond. Matter 8, 10049 (1996).

196 P.D. Johnson, T. Valla, A. Fedorov, Z. Yusof, B.O. Wells, Q. Li, A.R. Moodenbaugh, G.D. Gu, N. Koshizuka, C.
Kendziora, Sha Jian, D.G. Hinks, Phys. Rev. Lett. 87, 177007 (2001).

197 E. Dagotto, A. Nazarenko, and M. Boninsegni, Phys. Rev. Lett. 73, 728 (1994); E. Dagotto, A. Nazarenko, and A. Moreo, Phys. Rev. Lett. 74, 310 (1995); A. Nazarenko, K.J.E. Vos, S. Haas, E. Dagotto, and R.J. Gooding, Phys. Rev. B, 51, 8676 (1995).

198 E. Schachinger, J. P. Carbotte, and F. Marsiglio, Phys. Rev. B 56, 2738 (1997).

${ }^{199}$ F. Marsiglio, T. Startseva, and J. P. Carbotte, Physics Lett. A 245, 172 (1998).

${ }^{200}$ F. Bouis, M. N. Kiselev, F. Onufrieva, and P. Pfeuty, condmat/9906369.

V. Yu Irkhin, A.A. Katanin and M.I Katsnelson, condmat/0102381 and references therein.

${ }^{202}$ S. Sachdev Quantum Phase Transitions, Cambridge Univ Press., Cambridge (1999).

203 S. Sachdev, A. V. Chubukov, A. Sokol, Phys. Rev. B 51, 14874 (1995).

204 M. Lavagna, Phil. Mag. B 81, 1469 (2001).

${ }^{205}$ E. Abrahams and C. Varma, Proc. Natl. Acad. of Sciences 97, 5714 (2000).

206 D. J. Scalapino and S.R. White, Phys. Rev. B 58, 1347 (1998); E. Demler and S.-C. Zhang, Nature, 396, 733 (1999), B. Janko, cond-mat/9912073.

207 P. W. Anderson, Phys. Rev. Lett.67, 2092 (1991).

208 C. M. Varma and E. Abrahams, Phys. Rev. Lett. 86, 4652 (2001).

209 R. B. Laughlin, G. Lonzarich, P. Monthoux, and D. Pines, Advances in Physics 50, 361 (2001).

210 J. Cerne, M. Grayson, D. C. Schmadel, G. S. Jenkins, H. D. Drew, R. Hughes, A. Dabkowski, J. S. Preston and P.-J. Kung, Phys. Rev. Lett. 84, 3418 (2000).

211 See, e.g., N. P. Ong inPhysical Properties of High Temperature Superconductors, ed. by D. M. Ginsberg (World Scientific, Singapore, 1990), Vol. 2.

${ }^{212}$ B. Stojkovic and D. Pines, Phys. Rev. Lett. 70, 811 (1996); Phys. Rev. B 55, 8576 (1997).

${ }^{213}$ Y. Zha, V. Barzykin and D. Pines, Phys. Rev. B 54, 7561 (1996).

214 Y. Ando, G. S. Boebinger, A. Passner, T. Kimura, and K. Kishio, Phys. Rev. Lett. 75, 4662 (1995).

${ }^{215}$ G. S. Boebinger, Y. Ando, A. Passner, T. Kimura, M. Okuya, J. Shimoyama, K. Kishio, K. Tamasaku, N. Ichikawa, and S. Uchida Phys. Rev. Lett. 77, 5417 (1996).

${ }^{216}$ S. Ono, Y. Ando, T. Murayama, F. F. Balakirev, J. B. Betts, and G. S. Boebinger, Phys. Rev. Lett. 85, 638 (2000).

217 H. Kontani, K. Kanki, K. Ueda, Phys. Rev. B 59, 14723 (1999)

${ }^{218}$ P. W. Anderson, Science, 235 (1987) 4793; Mater. Res. Bull., 8 (1973) 153.

${ }^{219}$ X.-G. Wen and P. A. Lee, Phys. Rev. Lett. 76503 (1996).

220 S. Kivelson, D.S. Rokhsar, and J. Sethna, Phys. Rev. B 35, 8865 (1987).

${ }^{221}$ I. Affleck and J. B. Marston, Phys. Rev. B 37, 3774 (1988).

${ }^{222}$ G. Kotliar and J. Liu, Phys. Rev. B 38, 5142 (1988).

${ }^{223}$ Y. Suzumura, Y. Hasegawa, and H. Fukuyama, J. Phys. Soc. Jpn. 57, 2768 (1988).

${ }^{224}$ G. Baskaran and P. W. Anderson, Phys. Rev. B 37, 580 
(1988).

${ }^{225}$ P. A. Lee and N. Nagaosa, Phys. Rev. B 46, 5621 (1992).

${ }^{226}$ I. Affleck, Z. Zou, T. Hsu, and P. W. Anderson, Phys. Rev. B. 38, 745 (1988).

${ }^{227}$ D. Poilblanc and Y. Hasegawa, Phys. Rev. B. 41, 6989 (1990).

${ }^{228}$ T. Senthil and M. P.A. Fisher, Phys. Rev. B 62, 7850 (2000).

2
${ }^{229}$ Ar. Abanov, B. Altshuler and A. V. Chubukov, unpublished.

${ }^{230}$ M. Vojta and S. Sachdev, Phys. Rev. Lett.83, 3916 (1999); N. Read and S. Sachdev, Phys. Rev. Lett. 62, 1694 (1989); Phys. Rev. B 42, 4568 (1990).

231 J. Schmalian, P. G. Wolynes, Phys. Rev. Lett. 85, 836 (2000). 